A sensitivity and adjoint calculus for discontinuous solutions of hyperbolic conservation laws with source terms

\author{
Stefan Ulbrich
}

March 2000

TR00-10

Department of Computational and Applied Mathematics - MS134

Rice University

6100 Main Street

Houston, Texas 77005-1892

USA 


\title{
A SENSITIVITY AND ADJOINT CALCULUS FOR DISCONTINUOUS SOLUTIONS OF HYPERBOLIC CONSERVATION LAWS WITH SOURCE TERMS
}

\author{
STEFAN ULBRICH *
}

\begin{abstract}
We present a sensitivity and adjoint calculus for the control of entropy solutions of scalar conservation laws with controlled initial data and source term. The sensitivity analysis is based on shift-variations which are the sum of a standard variation and suitable corrections by weighted indicator functions approximating the movement of the shock locations. Based on a first order approximation by shift-variations in $L^{1}$ we introduce the concept of shift-differentiability which is applicable to operators having functions with moving discontinuities as images and implies differentiability for a large class of tracking-type functionals. In the main part of the paper we show that entropy solutions are generically shift-differentiable at almost all times $t>0$ with respect to the control. Hereby we admit shift-variations for the initial data which allows to use the shift-differentiability result repeatedly over time slabs. This is useful for the design of optimization methods with time domain decomposition. Our analysis, especially of the shock sensitivity, combines structural results by using generalized characteristics and an adjoint argument. Our adjoint based shock sensitivity analysis does not require to restrict the richness of the shock structure a priori and admits shock generation points. The analysis is based on stability results for the adjoint transport equation with discontinuous coefficients satisfying a one-sided Lipschitz condition. As a further main result we derive and justify an adjoint representation for the derivative of a large class of tracking-type functionals.
\end{abstract}

Key words. optimal control, scalar conservation law, shock sensitivity, adjoint state, Féchet differentiability

AMS subject classifications. 35L65, 49J50, 49K20, 35R05, 35B37

1. Introduction. This paper is concerned with the development of a sensitivity and adjoint calculus for the optimal control of entropy solutions of scalar conservation laws with source terms: Consider the Cauchy problem for an inhomogeneous conservation law

$$
\begin{aligned}
& \left.\partial_{t} y+\partial_{x} f(y)=g\left(t, x, y, u_{1}\right), \quad(t, x) \in \Omega_{T} \stackrel{\text { def }}{=}\right] 0, T[\times \mathbb{R} \\
& y(0, x)=u_{0}(x), \quad x \in \mathbb{R},
\end{aligned}
$$

where the flux $f: \mathbb{R} \longrightarrow \mathbb{R}$ is twice continuously differentiable, $u=\left(u_{0}, u_{1}\right) \in L^{\infty}(\mathbb{R}) \times$ $L^{\infty}\left(\Omega_{T}\right)^{m} \stackrel{\text { def }}{=} U$ is the control and $g: \Omega_{T} \times \mathbb{R} \times \mathbb{R}^{m} \rightarrow \mathbb{R}$ satisfies a growth condition (A1) $g \in L^{\infty}\left(\Omega_{T} ; C_{\text {loc }}^{0,1}\left(\mathbb{R} \times \mathbb{R}^{m}\right)\right)$ and for all $M_{u}>0$ there are $C_{1}, C_{2}>0$ with

$$
g\left(t, x, y, u_{1}\right) \operatorname{sgn}(y) \leq C_{1}+C_{2}|y|, \quad \forall\left(t, x, y, u_{1}\right) \in \Omega_{T} \times \mathbb{R} \times\left[-M_{u}, M_{u}\right]^{m} .
$$

Then one can show (e.g. [17,24]) that (1.1) admits for all $u \in U$ a unique entropy solution $y=y(u) \in L^{\infty}\left(\Omega_{T}\right) \cap C\left([0, T] ; L_{l o c}^{1}(\mathbb{R})\right)$. It is well known that in general weak solutions of (1.1) develop discontinuities after finite time and that uniqueness holds only in the class of entropy solutions. We recall that for given $u \in U$ a function $y=y(u) \in L^{\infty}\left(\Omega_{T}\right)$ is an entropy solution to (1.1) in the sense of Kružkov [17] if it satisfies the entropy inequality

$$
\partial_{t} \eta(y)+\partial_{x} q(y) \leq \eta^{\prime}(y) g\left(t, x, y, u_{1}\right) \quad \text { in } \mathcal{D}^{\prime}\left(\Omega_{T}\right)
$$

for all convex functions (entropies) $\eta: \mathbb{R} \rightarrow \mathbb{R}$ with corresponding entropy fluxes $q(y)=$ $\int_{0}^{y} \eta^{\prime}(s) f^{\prime}(s) d s$ and the initial condition in the sense

$$
\left.\underset{t \rightarrow 0+}{\operatorname{ess}} \lim _{t \rightarrow y} \| t, \cdot\right)-u_{0} \|_{1, K} d \tau=0 \quad \forall K \subset \subset \mathbb{R} .
$$

The aim of this paper is to develop and justify - without a priori assumptions on the shock structure - a sensitivity calculus for the control-to-state mapping $u \longmapsto y(u)$ that yields in

*Zentrum Mathematik, Technische Universität München, 80290 München, Germany (sulbrich@ma . tum. de). The author was supported by Deutsche Forschungsgemeinschaft under Grant U1158/2-1 and by CRPC grant CCR9120008 . 
particular the differentiability and a formula for the derivative of objective functionals

$$
J(y(u))=\int_{a}^{b} \phi\left(y(\bar{t}, \cdot ; u), y_{d}\right) d x
$$

with data $y_{d} \in B V([a, b]), \phi \in C_{l o c}^{1,1}\left(\mathbb{R}^{2}\right)$ and $\left.\left.\bar{t} \in\right] 0, T\right]$. Moreover, we will derive an adjoint formula for the gradient of (1.2). The adjoint equation is a transport equation with source term and its coefficient is discontinuous along shock curves which requires a careful definition of the adjoint state as a reversible solution to ensure uniqueness and stability. These results are useful for the design of gradient based methods for the solution of control problems of the type

$$
\min \tilde{J}(y(u), u) \stackrel{\text { def }}{=} J(y(u))+R(u) \quad \text { subject to } u \in U_{a d}, y(u) \text { solves (1.1). }
$$

In [24] we have derived results on the existence of optimal solutions for $(\mathrm{P})$ and the convergence of discretized approximations for the multidimensional case. For example, $(\mathrm{P})$ has an optimal solution if $U_{a d}$ is bounded in $L^{\infty}(\mathbb{R}) \times L^{\infty}\left(\Omega_{T}\right)^{m}$ and compact in $L_{l o c}^{1}(\mathbb{R}) \times L_{l o c}^{1}\left(\Omega_{T}\right)^{m}$ and if $\tilde{J}: C\left([0, T] ; L_{l o c}^{r}(\mathbb{R})\right) \times\left(U_{a d} \subset L_{l o c}^{r}(\mathbb{R}) \times L_{l o c}^{r}\left(\Omega_{T}\right)^{m}\right) \longrightarrow \mathbb{R}$ is sequentially lower semicontinuous for some $r \in[1, \infty[$. Moreover, for the present one dimensional case existence results without compactness assumption on $U_{a d}$ were obtained in [24] using compensated compactness.

The state equation (1.1) is a useful model for the study of control problems involving flows with shocks. In particular, it is shown in [10] that the steady flow of an inviscid fluid in a duct governed by the Euler equations can be reduced to determining the velocity $y$ as a steady state solution of (1.1) for $f(y)=y+2 \bar{\gamma} H / y, g\left(x, y, u_{1}\right)=u_{1} \bar{\gamma}(y-2 H / y)$, where $H$ is the total enthalpy, $\bar{\gamma}=(\gamma-1) /(\gamma+1)$ with the gas constant $\gamma>1$ and the design variable $u_{1}=\partial_{x} A / A, A(x)$ being the cross-sectional area of the duct. Moreover, it is noted in [10] that the corresponding time-dependent problem (1.1) captures some essential features of the time-dependent Euler equations and is therefore a suitable model problem for the study of unsteady duct flows with shocks. Since the flow over a transonic airfoil is qualitatively similar to one-dimensional duct flows, the study of the differentiability properties of (1.1)-(1.2) is thus useful to gain insight into the optimal design of airfoils under unsteady flow conditions. In particular, the sensitivity of flows with shocks with respect to time-dependent changes of the geometry is of practical importance for the control of systems with fluid-structure coupling, e.g. the fluttering problem of airfoils.

In this work we give a rigorous sensitivity analysis and adjoint calculus for solutions of (1.1) with shocks and thereby provide an analytical framework for the study and numerical solution of optimal control problems governed by hyperbolic balance laws (1.1). The main features of our approach are:

- we derive a sensitivity calculus based on shift-variations that implies the Fréchet differentiability for objective functionals (1.2),

- we give and rigorously justify a gradient representation for objective functionals (1.2) by using an appropriately defined adjoint state,

- the shock structure has not to be restricted a priori and shock generation points are allowed,

- we admit non-entropy-admissible initial data and allow shift-variations of the initial data that move shock locations.

The crucial part is the analysis of the shock sensitivities. In our approach we derive the differentiability of a shock position $x_{s}$ at time $\bar{t}$ by analyzing the smoothness properties of the functional $u \longmapsto \int_{x_{s}-\varepsilon}^{x_{s}-\varepsilon} y(\bar{t}, x ; u) d x$ with the help of an adjoint calculus for $\varepsilon \rightarrow 0$. The adjoint argument is mainly based on the stability of the adjoint equation with respect to its coefficients. Then the properties of $u \longmapsto x_{s}(u)$ follow from basic stability properties of the shock that we derive a priori by using the theory of generalized characteristics. An advantage of this method lies in the fact that the shock structure of the solution has not to be restricted 
a priori. In particular, shock generation points are allowed. This approach can, at least in a formal manner, also be applied to hyperbolic systems and gives the correct shock sensitivity if the necessary stability properties of the shock and the adjoint equation actually hold.

It can be shown (see e.g. [24] for the problem at hand) that the mapping $u \in U \longmapsto y(u) \in$ $C\left([0, T] ; L_{\text {loc }}^{1}(\mathbb{R})\right)$ is locally Lipschitz, but very simple examples show that this mapping is in general not directionally differentiable if $y(u)$ contains shocks, even if $L^{\infty}$ is replaced by $C^{\infty}$ in the definition of $U$. This is caused by the fact that a variation of $u$ results in a shift of the shocks, which can not be approximated appropriately in the linear structure of $L_{l o c}^{1}$. Consider for example the following family of Riemann problems for the inviscid Burgers equation

$$
\partial_{t} y_{\varepsilon}+\partial_{x} \frac{y_{\varepsilon}^{2}}{2}=0, \quad y_{\varepsilon}(0, x)=u_{0}(x)+\varepsilon \delta u_{0}(x) \stackrel{\text { def }}{=} \begin{cases}1+\varepsilon & \text { if } x \leq 0 \\ -1 & \text { if } x>0\end{cases}
$$

The solution has a shock $\eta_{\varepsilon}$ emanating from $(0,0)$ with shock speed

$$
\dot{\eta}_{\varepsilon}(t)=\frac{\left[\left(y_{\varepsilon}\left(t, \eta_{\varepsilon}(t)\right)\right)^{2} / 2\right]}{\left[y_{\varepsilon}\left(t, \eta_{\varepsilon}(t)\right)\right]}=\frac{\varepsilon}{2},
$$

according to the jump condition, where $[h(t, x)]=h(t, x-)-h(t, x+)$ denotes the jump of $h(t, \cdot)$ across $x$. Thus, we have $\eta_{\varepsilon}(t)=\frac{\varepsilon}{2} t$ and the corresponding entropy solution

$$
y_{\varepsilon}= \begin{cases}1+\varepsilon & \text { if } x \leq t \varepsilon / 2 \\ -1 & \text { if } x>t \varepsilon / 2\end{cases}
$$

Of course, the Lipschitz continuous map $\varepsilon \longmapsto y_{\varepsilon}(t, \cdot) \in L_{l o c}^{1}(\mathbb{R})$ is not differentiable, since the difference quotient does only converge in a weaker topology, e.g. weakly in the space $\mathcal{M}_{\text {loc }}(\mathbb{R})$ of locally bounded Borel measures. In fact, $\varepsilon \longmapsto y_{\varepsilon}(t, \cdot) \in \mathcal{M}_{\text {loc }}(\mathbb{R})$ is differentiable in the weak topology and we have for example at $\varepsilon=0$

$$
\left.\frac{d}{d \varepsilon} y_{\varepsilon}(t, \cdot)\right|_{\varepsilon=0} \varepsilon=\mathbf{1}_{\left\{x<\eta_{0}(t)\right\}} \varepsilon+\left[y_{0}\left(t, \eta_{0}(t)\right)\right] \delta_{\eta_{0}(t)} \frac{t}{2} \varepsilon
$$

where $\mathbf{1}_{I}$ denotes the indicator function of a set $I$, i.e. $\mathbf{1}_{I}(x)=1$ if $x \in I, \mathbf{1}_{I}(x)=0$ else, and $\delta_{x}$ denotes the Dirac measure located at $x$. Hereby, $\frac{t}{2} \varepsilon$ is a linear (in this case exact) approximation of the actual shock shift $\eta_{\varepsilon}(t)-\eta_{0}(t)$. Note however, that a differentiability result in the weak topology of $\mathcal{M}_{\text {loc }}(\mathbb{R})$ is not strong enough to derive the differentiability of the functional (1.2) without additional structural information. To get a first order approximation in $L_{l o c}^{1}$, we have to leave the linear structure of $L_{l o c}^{1}$ in order to allow for an accurate approximation of the shock movement. A natural way to achieve this is to replace the singular (second) part of the measure (1.3) by the function $\operatorname{sgn}\left(\frac{t}{2} \varepsilon\right)\left[y_{0}\left(t, \eta_{0}(t)\right)\right] \mathbf{1}_{I\left(\eta_{0}(t), \eta_{0}(t)+\frac{t}{2} \varepsilon\right)}$, where $I(a, b) \stackrel{\text { def }}{=}[\min \{a, b\}, \max \{a, b\}]$ and $\operatorname{sgn}(\cdot)$ is the sign function. We thereby obtain a first order approximation of $y_{\varepsilon}(t)-y_{0}(t)$ in $L_{l o c}^{1}$ by the shift variation

$$
S_{y_{0}(t)}^{\eta_{0}(t)}\left(\mathbf{1}_{\left\{x<\eta_{0}(t)\right\}} \varepsilon, \frac{t}{2} \varepsilon\right) \stackrel{\text { def }}{=} \mathbf{1}_{\left\{x<\eta_{0}(t)\right\}} \varepsilon+\operatorname{sgn}\left(\frac{t}{2} \varepsilon\right)\left[y_{0}\left(t, \eta_{0}(t)\right)\right] \mathbf{1}_{I\left(\eta_{0}(t), \eta_{0}(t)+\frac{t}{2} \varepsilon\right)} .
$$

In this paper we will develop a sensitivity calculus based on shift-variations for the mapping $u \longmapsto y(\bar{t}, \cdot ; u), \bar{t} \in[0, T]$, defined in (1.1) in the case $f^{\prime \prime} \geq m_{f^{\prime \prime}}>0$. Let piecewise $C^{1}$ initial data $u_{0} \in P C^{1}\left(\mathbb{R} ; x_{1}, \ldots ; x_{N}\right)$ and $u_{1} \in L^{\infty}\left(0, T ; C^{1}(\mathbb{R})^{m}\right)$ be given. Using the theory of generalized characteristics [8] we will show that for a given interval $I$ and time $\bar{t}>0$ the following situation is generic: $y(\bar{t}, \cdot ; u)$ has on $I$ finitely many shocks at $\bar{x}_{1}<\ldots<\bar{x}_{K}$, the shock locations depend differentiable on $u$ and the states connected by the shocks vary 
differentiable in the strong topology of $L^{1}$. From this we will deduce that the variation $y(\bar{t}, \cdot ; u+\delta u)-y(\bar{t}, \cdot ; u)$ allows a first order approximation by a shift variation of the form

$$
S_{y(\bar{t}, ; u)}^{\left(\bar{x}_{k}\right)}(\delta y, \bar{s}) \stackrel{\text { def }}{=} \delta y+\sum_{k}\left[y\left(\bar{t}, \bar{x}_{k} ; u\right)\right] \operatorname{sgn}\left(\bar{s}_{k}\right) \mathbf{1}_{I\left(\bar{x}_{k}, \bar{x}_{k}+\bar{s}_{k}\right)}
$$

where $(\delta y, \bar{s})$ depends linearly on $\delta u, \bar{x}_{k}$ are the shock locations, $\left[y\left(\bar{t}, \bar{x}_{k} ; u\right)\right]=y\left(\bar{t}, \bar{x}_{k}-; u\right)-$ $y\left(\bar{t}, \bar{x}_{k}+; u\right)$ denotes the jump across the shock, $I\left(\bar{x}_{k}, \bar{x}_{k}+\bar{s}_{k}\right)$ is the interval enclosed by the arguments, $\mathbf{1}_{I\left(\bar{x}_{k}, \bar{x}_{k}+\bar{s}_{k}\right)}$ is its indicator function, and $\bar{s}_{k}$ is a linear approximation of the shock shift. To mimic the behavior of $y(\cdot ; u)$ at later times, it is natural to go one step further and to admit shift-variations already for the initial data. Roughly speaking, we will in particular show that for $\left(u_{0}, u_{1}\right)$ as above, $W \stackrel{\text { def }}{=} P C^{1}\left(\mathbb{R} ; x_{1}, \ldots, x_{N}\right) \times L^{\infty}\left(0, T ; C^{1}(\mathbb{R})^{m}\right) \times \mathbb{R}^{N}$ and almost all $\bar{t}$ the mapping

$$
\left(w_{0}, w_{1}, \sigma\right) \in W \longmapsto y\left(\bar{t}, \cdot ; u_{0}+S_{u_{0}}^{\left(x_{i}\right)}\left(w_{0}, \sigma\right), u_{1}+w_{1}\right) \in L^{1}(I)
$$

is shift differentiable with respect to $\left(w_{0}, w_{1}, \sigma\right)$ at 0 in the sense that its variation admits a first order approximation by a shift variation of the form (1.4), where $(\delta y, \bar{s})$ depends linearly on $\delta w_{0}, \delta w_{1}$, and $s=\delta \sigma$. Hereby, $\delta y$ can be obtained as the trace $\delta Y(\bar{t})$ of a function $\delta Y$ that is the piecewise solution of the linearization of (1.1) outside of the shock set and $\bar{s}_{k}$ can be obtained by an adjoint formula. We admit shift variations of the initial data since this allows to use the shift-differentiability result repeatedly over time slabs. This is helpful for the design of optimization algorithms with time domain decomposition for the solution of (P). By introducing a general concept of shift-differentiability we will be able to derive results on the Fréchet differentiability of tracking-type functionals of the form (1.2) as long as the discontinuities of $y_{d}$ and $y(\bar{t}, \cdot ; u)$ do not coincide. If $y_{d}$ and $y(\bar{t}, \cdot ; u)$ share discontinuities we will still obtain directional differentiability. For objective functionals of the form (1.2) we will derive a gradient representation via an adjoint state. The proper definition of the adjoint state requires an extension of the concept of reversible solutions of backward transport equations with discontinuous coefficients introduced in [1] to the case

$$
\begin{aligned}
& \left.\partial_{t} p+f^{\prime}(y) \partial_{x} p=-g_{y}\left(t, x, y, u_{1}\right) p, \quad(t, x) \in \Omega_{\bar{t}} \stackrel{\text { def }}{=}\right] 0, \bar{t}[\times \mathbb{R} \\
& p(\bar{t}, x)=p^{\bar{t}}(x), \quad x \in \mathbb{R}
\end{aligned}
$$

with linear source term, where $p^{\bar{t}}$ are suitable end data.

The results of this paper can be straightforward extended to identification problems for the flux $f$, where $f$ is the control. Identification problems of this type are considered by James and Sepúlveda [16]. The differentiability of the objective function for the hyperbolic case was left open. The techniques of the present paper can be used to obtain a sensitivity and adjoint calculus for flux identification as well.

In recent years several results on sensitivities and adjoints for hyperbolic conservation laws were obtained by other authors $[1-6,11,12]$ but most results assume a priori knowledge of the shock structure (usually one shock separating smooth states) or are restricted to the conservative case $g \equiv 0$. The conservative case admits special techniques, since the characteristics are straight lines and the solution can be represented by the integral formula of Lax [18]. Bouchut and James apply in [2] their existence and stability results of [1] for measure-valued duality solutions of linear conservation laws with discontinuous coefficients to derive for the case $g \equiv 0, f^{\prime \prime}>0$ that $u_{0} \in L^{\infty} \longmapsto y(\cdot ; u) \in C\left([0, T] ; \mathcal{M}_{l o c}(\mathbb{R})-\mathrm{w}^{*}\right)$ is directionally differentiable at an entropy-admissible $u_{0}$ where the space $\mathcal{M}_{\text {loc }}(\mathbb{R})$ of local Borel measures is equipped with the usual weak topology. Note that this topology is too weak to derive directly differentiability results for (1.2) without using additional structural information. Godlewski and Raviart study in [11] (see also [12]) the linearized stability of multidimensional hyperbolic 
systems of conservation laws for perturbations of the initial data of a base solution with a one dimensional shock. They define measure solutions for the linearized equations with singular part along the shock and construct numerical schemes for the solution of the linearized problem. For the conservative scalar problem with Riemann initial data it is shown that the linearization coincides with the first order expansion in $C\left([0, T] ; \mathcal{M}_{l o c}(\mathbb{R})-\mathrm{w}^{*}\right)$ of [2]. In this paper we give further justification of this linearization process for more general situations. Bouchut and James develop in [1] existence and stability results for transport equations with discontinuous coefficients satisfying a one sided Lipschitz condition that will be extended in the present work for the analysis of the adjoint equation (1.6). Previous results on the adjoint equation were obtained in the context of uniqueness results in $[7,15,19,20]$, and of error estimates for approximate solutions in [23]. In [19] adjoint equations for a class of systems of conservation laws are considered. An extension of our approach to systems seems to be possible by building on this work. In [3] a new differential structure on the space BV obtained by horizontal shifts of the points of the graph is introduced and it is shown that in the case $g \equiv 0, f^{\prime \prime}>0$ the flow $u_{0} \in L^{\infty} \longmapsto y(t, \cdot ; u)$ generated by (1.1) is generically differentiable w.r.t. this structure. The analysis uses the integral formula of Lax. Bressan and Marson [4] use generalized tangent vectors to develop a variational calculus for piecewise Lipschitz solutions of systems of conservation laws. Using our notation (1.4), they show that for piecewise Lipschitz initial data $u_{0}^{\varepsilon}, \varepsilon \geq 0$, such that $u_{0}^{\varepsilon}-u_{0}=S_{u_{0}}^{\left(x_{i}\right)}\left(\varepsilon \delta u_{0}, \varepsilon s\right)+o(\varepsilon)$ in $L_{l o c}^{1}$, the corresponding solutions $y_{\varepsilon}$ satisfy $y_{\varepsilon}(\bar{t}, \cdot)-y(\bar{t}, \cdot)=S_{y(\bar{t}, \cdot)}^{\left(\bar{x}_{k}\right)}(\varepsilon \delta y, \varepsilon \bar{s})+o(\varepsilon)$ in $L_{l o c}^{1}$ if $\bar{t}$ is so small that $y_{\varepsilon}$ remains piecewise Lipschitz on $[0, \bar{t}]$. While this result applies to systems it considers only directional variations and requires the structural assumption of piecewise Lipschitz solutions which is not needed in the present paper. Especially the analysis of the shock sensitivity differs significantly from our approach, since in [4] the linearized Rankine-Hugoniot jump condition together with the linearized state equation is used to derive an ODE for the shock sensitivity, while we use an adjoint formula which reduces the necessary structural information on the history of the shock as far as possible. Moreover, we develop an adjoint calculus that gives a gradient representation for objective functionals (1.2). Cliff, Heinkenschloss, and Shenoy study in [5,6] design problems for one-dimensional steady duct flow. By introducing the single shock location as additional state variable and transforming the space variable such that the shock location is fixed Fréchet differentiability is shown. Optimality conditions are derived and an adjoint-based gradient representation of the objective function is given. Finally, numerical results for the application of an SQP method to the discretized problem are reported.

This paper is organized as follows. In section 2 we introduce the concept of shift-differentiability for operators having discontinuous functions with moving discontinuities as images which is based on a first order approximation by shift-variations (1.4). Moreover, we will show that the superposition (1.2) of a shift-differentiable operator $u \longmapsto y(\bar{t}, \cdot ; u)$ and a tracking-type functional is Fréchet differentiable if $y_{d}, y(\bar{t}, \cdot ; u)$ do not share discontinuities and is directionally differentiable else. The shift-differentiability of the control-state-mapping $u \longmapsto y(\bar{t}, \cdot ; u)$ for entropy solutions of (1.1) is analyzed in sections 3-10. Section 3 provides the necessary stability results and collects structural results of [8] provided by the theory of generalized characteristics. In section 4 continuity points are analyzed that are neither shock generation points nor located on the boundary of rarefaction waves. In section 5 the stability of shocks and the differentiability of the shock location at a time $\bar{t}>0$ is shown under a nondegeneracy assumption. The proof of the latter is carried out in section 7 , since it requires stability results for the adjoint equation which are provided in section 6 . In section 8 continuity points are analyzed that are located on characteristics emanating from points where discontinuities are produced under shift-variations. Section 9 studies points on the boundary of rarefaction waves. Section 10 combines this to prove as a main result the shift-differentiability of entropy solutions at time $\bar{t}>0$ w.r.t the controls if the nondegeneracy assumption holds for all shocks at time $\bar{t}$. 
Moreover, a differentiability result for objective functionals (1.2) is obtained by using section 2. In section 11 an adjoint formula for the derivative of these objective functionals is derived. Moreover, it is shown that the required nondegeneracy assumption holds for all shocks at almost all times $\bar{t}>0$.

Notations. For Lebesgue-measurable $S \subset \mathbb{R}^{n}$ the norm of the Lebesgue-spaces $L^{r}(S)$, $1 \leq r \leq \infty$, is denoted by $\|\cdot\|_{r, S}$. In the case $S=\mathbb{R}^{n}$ we write $\|\cdot\|_{r}$. By $(\cdot, \cdot)_{2, S}$ we denote the inner product on $L^{2}(S)$. For an interval $I \subset \mathbb{R}$ the space of functions $v \in L^{1}(I)$ with bounded variation $|v|_{v a r}$ is denoted by $B V(I)$. For open $S \subset \mathbb{R}^{n}$ we mean by $C^{k}(S)$, $k \in \mathbb{N}_{0}$, the space of functions with continuous, bounded derivatives on $S$ up to order $k$ equipped with the usual norm $\|v\|_{C^{k}(S)}=\sum_{|\beta| \leq k}\left\|D^{\beta} v\right\|_{\infty, S} . C^{k}\left(S^{c l}\right)$ is the subspace of functions in $C^{k}(S)$ that admit a continuous extension of the first $k$ derivatives to $S^{c l}$. Moreover, we write $C(S)$ instead of $C^{0}(S)$. $C^{k, \alpha}\left(S^{c l}\right), 0<\alpha \leq 1$, is the usual Hölder space. For closed $I \supset[a, b], a<b$, we denote by $P C^{k}\left(I ; x_{1}, \ldots, x_{N}\right)$ the space of piecewise $C^{k}$-functions $v$ with possible discontinuities at $a<x_{1}<\ldots<x_{N}<b$, more precisely $\left.v\right|_{I_{i}} \in C^{k}\left(I_{i}^{c l}\right)$, $i=0, \ldots, N$, with $\left.I_{i}=\right] x_{i}, x_{i+1}\left[, i=1, \ldots, N-1, I_{0}=I \cap\left\{x<x_{1}\right\}, I_{N}=I \cap\left\{x>x_{N}\right\}\right.$. It is endowed with the norm $\|v\|_{P C^{k}\left(I ; x_{1}, \ldots, x_{n}\right)}=\sum_{i=0}^{N}\|v\|_{C^{k}\left(I_{i}^{c l}\right)}$. The indicator function of a set $I$ is denoted by $\mathbf{1}_{I}$, i.e. $\mathbf{1}_{I}(x)=1$ if $x \in I$ and $\mathbf{1}_{I}(x)=0$ else.

2. Shift-differentiability. In this section we introduce a concept of shift-differentiability that yields an extension of classical differentiability to operators $u \longmapsto y(u) \in L_{l o c}^{1}(\mathbb{R})$ having functions with moving discontinuities as images. It is based on shift-variations that are the sum of a standard variation and suitably scaled indicator functions approximating the actual shift of discontinuities. The interesting point is that the shift-differentiability of an operator implies under quite general circumstances the Fréchet differentiability of tracking-type functionals analogously to (1.2). As motivated in the introduction we define shift-variations as follows.

Definition 2.1. Let $I=[a, b], a<b$ and let $w \in B V(I)$. Given $a<x_{1}<x_{2}<$ $\ldots<x_{N}<b$, we call $(\delta w, s) \in L^{\infty}(I) \times \mathbb{R}^{N}$ a generalized variation of $w$ and associate with it the shift-variation $S_{w}^{\left(x_{i}\right)}(\delta w, s) \in L^{\infty}(\mathbb{R})$ of $w$ by

$$
S_{w}^{\left(x_{i}\right)}(\delta w, s)(x) \stackrel{\text { def }}{=} \delta w(x)+\sum_{i=1}^{N}\left[w\left(x_{i}\right)\right]_{+} \operatorname{sgn}\left(s_{i}\right) \mathbf{1}_{I\left(x_{i}, x_{i}+s_{i}\right)}(x)
$$

where $\left[w\left(x_{i}\right)\right]_{+} \stackrel{\text { def }}{=} \max \left\{0, w\left(x_{i}-\right)-w\left(x_{i}+\right)\right\}$ and $I(\alpha, \beta) \stackrel{\text { def }}{=}[\min \{\alpha, \beta\}, \max \{\alpha, \beta\}]$ for $\alpha, \beta \in \mathbb{R}$.

The restriction that only down-jumps are shifted is motivated by the fact that entropy solutions of (1.1) satisfy Oleinik's entropy condition $y(t, x-) \geq y(t, x+)$ for all $x \in \mathbb{R}$ and a.a. $t \in] 0, T]$, see section 3.1 below.

We call an operator shift-differentiable, if its actual variation admits a first order approximation in $L_{l o c}^{1}$ by a shift-variation, more precisely

DeFinition 2.2. Let $U$ be a real Banach space and $I=[a, b], a<b$. For an open subset $D \subset U$ let $u \in D \longmapsto y(u) \in L^{\infty}(\mathbb{R})$ be locally bounded. Moreover, let $\bar{u} \in D$ with $y(\bar{u}) \in B V(I)$. We say that $y$ is shift-differentiable at $\bar{u}$ if there are $a<\bar{x}_{1}<\bar{x}_{2}<\ldots<$ $\bar{x}_{K}<b$ and a bounded linear operator $\left.\left.T_{s}(\bar{u})=D_{s} y(\bar{u}) \in \mathcal{L}\left(U, L^{r}(I) \times \mathbb{R}^{K}\right), r \in\right] 1, \infty\right]$, such that

$$
\lim _{u \rightarrow \bar{u}} \frac{\left\|y(u)-y(\bar{u})-S_{y(\bar{u})}^{\left(\bar{x}_{k}\right)}\left(T_{s}(\bar{u}) \cdot(u-\bar{u})\right)\right\|_{1, I}}{\|u-\bar{u}\|_{U}}=0 .
$$

We say that $y$ is continuously shift-differentiable at $\bar{u}$ ify is shift-differentiable in a neighborhood of $\bar{u}$ and if the corresponding $T_{s}(u), \bar{x}_{k}(u), k=1, \ldots, K$, as well as $y(u)\left(\bar{x}_{k}(u) \pm\right)$ are continuous at $\bar{u}$. 
The property of shift-differentiability is strong enough that it implies the Fréchet differentiability of functionals of the form

$$
J(y(u)) \stackrel{\text { def }}{=} \int_{a}^{b} \phi\left(y(u)(x), y_{d}(x)\right) d x
$$

under quite moderate assumptions on $\phi$ and $y_{d}$.

LemMa 2.3. Let $u \longmapsto y(u)$ be shift-differentiable in $\bar{u}$ according to Definition 2.2. Moreover, let $y_{d} \in L^{\infty}(I)$ be approximately continuous at $\bar{x}_{1}, \ldots, \bar{x}_{K}$. Then for any function $\phi \in C_{\text {loc }}^{1,1}\left(\mathbb{R}^{2}\right)$ the functional $u \in U \longmapsto J(y(u))$ given by (2.1) is Fréchet differentiable at $\bar{u}$ and with $(\delta y, \bar{s}) \stackrel{\text { def }}{=} T_{s}(\bar{u}) \cdot \delta u, \bar{y} \stackrel{\text { def }}{=} y(\bar{u})$ holds

$$
\begin{aligned}
d_{u} J(\bar{y}) \cdot \delta u= & \left(\phi_{y}\left(\bar{y}, y_{d}\right), \delta y\right)_{2, I} \\
& +\sum_{k=1}^{K} \int_{0}^{1} \phi_{y}\left(\bar{y}\left(\bar{x}_{k}+\right)+\tau\left[\bar{y}\left(\bar{x}_{k}\right)\right], y_{d}\left(\bar{x}_{k}\right)\right) d \tau\left[\bar{y}\left(\bar{x}_{k}\right)\right]_{+} \bar{s}_{k} .
\end{aligned}
$$

If $y$ is continuously shift-differentiable at $\bar{u}$ and $y_{d}$ is continuous in a neighborhood of $\bar{x}_{1}, \ldots, \bar{x}_{K}$ then $u \longmapsto J(y(u))$ is continuously Fréchet differentiable at $\bar{u}$.

If at least one $\bar{x}_{k}$ is an approximate discontinuity of $y_{d}$ then $J(y(u))$ is still directionally differentiable and with $(\delta y, \bar{s})=T_{s}(\bar{u}) \cdot \delta u$ the directional derivative $\delta_{u}(J(y(\bar{u})) ; \delta u)$ is given by (2.2) if $y_{d}\left(\bar{x}_{k}\right)$ is replaced by $y_{d}\left(\bar{x}_{k}+0 \cdot \operatorname{sgn}\left(\bar{s}_{k}\right)\right)$.

Proof. Obviously, it is sufficient to consider the case $K=1$. We set $\bar{x}=\bar{x}_{1}$. Let $B \subset U$ be a bounded neighborhood of 0 such that $\bar{u}+B \subset D$. Then $\|y(\bar{u}+\delta u)\|_{\infty} \leq M_{y}$ for all $\delta u \in B$. In the sequel we will frequently use the abbreviation $u=\bar{u}+\delta u$ for $\delta u \in B$. Moreover, we will write $y, \bar{y}$ instead of $y(u), y(\bar{u})$ and set $(\delta y, \bar{s})=T_{s}(\bar{u}) \cdot \delta u$.

Since $y(u)$ is shift-differentiable in $\bar{u}$, we have for all $\delta u \in B$

$$
\|y-\bar{y}\|_{1} \leq o\left(\|\delta u\|_{U}\right)+\left\|S_{\bar{y}}^{\bar{x}}(\delta y, \bar{s})\right\|_{1} \leq o\left(\|\delta u\|_{U}\right)+\|\delta y\|_{1}+2 M_{y}|\bar{s}| \leq C_{1}\|\delta u\|_{U} .
$$

Let $L$ be a Lipschitz constant of $\phi_{y}$ w.r.t. $y$ on $\left[-M_{y}, M_{y}\right] \times\left[-\left\|y_{d}\right\|_{\infty},\left\|y_{d}\right\|_{\infty}\right]$. Set

$$
\bar{\phi}_{y}(\delta u) \stackrel{\text { def }}{=} \int_{0}^{1} \phi_{y}\left(\tau \bar{y}+(1-\tau) y, y_{d}\right) d \tau
$$

Then $\left\|\bar{\phi}_{y}(\delta u)\right\|_{\infty} \leq C_{2}, C_{2}>0$, for all $\delta u \in B$ and

$$
\left|J(y)-J(\bar{y})-\left(\bar{\phi}_{y}(\delta u), S_{\bar{y}}^{\bar{x}}(\delta y, \bar{s})\right)_{2, I}\right| \leq\left\|\bar{\phi}_{y}(\delta u)\right\|_{\infty}\left\|y-\bar{y}-S_{\bar{y}}^{\bar{x}}(\delta y, \bar{s})\right\|_{1}=o\left(\|\delta u\|_{U}\right) .
$$

To compare the last term on the left hand side with (2.2) we note that

$$
\left(\bar{\phi}_{y}(\delta u), S_{\bar{y}}^{\bar{x}}(\delta y, \bar{s})\right)_{2, I}=\left(\bar{\phi}_{y}(\delta u), \delta y\right)_{2, I}+[\bar{y}(\bar{x})]_{+} \operatorname{sgn}(\bar{s})\left(\bar{\phi}_{y}(\delta u), \mathbf{1}_{I(\bar{x}, \bar{x}+\bar{s})}\right)_{2, I} .
$$

For the first term we have with $1 / r+1 / r^{\prime}=1$ the estimate

$$
\begin{aligned}
\left\|\bar{\phi}_{y}(\delta u) \delta y-\phi_{y}\left(\bar{y}, y_{d}\right) \delta y\right\|_{1} & \leq\left\|\bar{\phi}_{y}(\delta u)-\phi_{y}\left(\bar{y}, y_{d}\right)\right\|_{r^{\prime}}\|\delta y\|_{r} \\
& \leq L\|y-\bar{y}\|_{r^{\prime}}\left\|T_{s}(\bar{u})\right\|\|\delta u\|_{U}=o\left(\|\delta u\|_{U}\right),
\end{aligned}
$$

since the first factor tends to zero by interpolation using (2.3) and the $L^{\infty}$-bound.

If $[\bar{y}(\bar{x})]_{+}=0$ then the second term in (2.4) vanishes and since $S_{\bar{y}}^{\bar{x}}(\delta y, \bar{s})=\delta y$ in this case, the proof is complete. Otherwise, we have $[\bar{y}(\bar{x})]_{+}=[\bar{y}(\bar{x})]$. To approximate the second term in (2.4) we observe that with $\delta \bar{y} \stackrel{\text { def }}{=} \max \left\{\min \left\{\delta y, 2 M_{y}\right\},-2 M_{y}\right\}$ obviously also holds

$$
\left\|y-\bar{y}-S_{\bar{y}}^{\bar{x}}(\delta \bar{y}, \bar{s})\right\|_{1}=o\left(\|\delta u\|_{U}\right) .
$$


Since the function on the left hand side has a uniform $L^{\infty}$-bound, we obtain with the local Lipschitz continuity of $\phi_{y}$

$$
\left\|\bar{\phi}_{y}(\delta u)-\int_{0}^{1} \phi_{y}\left(\bar{y}+\tau S_{\bar{y}}^{\bar{x}}(\delta \bar{y}, \bar{s}), y_{d}\right) d \tau\right\|_{1}=o\left(\|\delta u\|_{U}\right) .
$$

Finally, we have

$$
\begin{aligned}
& I_{1} \stackrel{\text { def }}{=}\left|\left(\int_{0}^{1} \phi_{y}\left(\bar{y}+\tau S_{\bar{y}}^{\bar{x}}(\delta \bar{y}, \bar{s}), y_{d}\right) d \tau, \mathbf{1}_{I(\bar{x}, \bar{x}+\bar{s})}\right)_{2}-\right| \bar{s}\left|\int_{0}^{1} \phi_{y}\left(\bar{y}(\bar{x}+)+\tau[\bar{y}(\bar{x})], y_{d}(\bar{x})\right) d \tau\right| \\
& =\left|\int_{\bar{x}}^{\bar{x}+\bar{s}} \int_{0}^{1}\left(\phi_{y}\left(\bar{y}+\tau[\bar{y}(\bar{x})] \operatorname{sgn}(\bar{s})+\tau \delta \bar{y}, y_{d}\right)-\phi_{y}\left(\bar{y}(\bar{x} \pm) \pm \tau[\bar{y}(\bar{x})], y_{d}(\bar{x})\right)\right) d \tau d x\right| .
\end{aligned}
$$

Since the arguments of $\phi_{y}$ are bounded, we get with a Lipschitz constant $L$

$$
\begin{aligned}
I_{1} & \leq L\left(\|\delta \bar{y}\|_{1, I(\bar{x}, \bar{x}+\bar{s})}+|\bar{s}|\left|\frac{1}{\bar{s}} \int_{\bar{x}}^{\bar{x}+\bar{s}}\left(|\bar{y}-\bar{y}(\bar{x}+0 \operatorname{sgn}(\bar{s}))|+\left|y_{d}-y_{d}(\bar{x})\right|\right) d x\right|\right) \\
& \leq L\|\delta y\|_{r}|\bar{s}|^{1 / r^{\prime}}+o(|\bar{s}|)=o\left(\|\delta u\|_{U}\right) .
\end{aligned}
$$

Hereby we have used that $y_{d}$ is approximately continuous in $\bar{x}$ and $y(\bar{u}) \in B V(I)$. Now the Fréchet differentiability of $J(y(u))$ and (2.2) follow by combining (2.4)-(2.7).

Now assume that $y_{d}$ is continuous in a neighborhood of $\bar{x}_{1}, \ldots, \bar{x}_{K}$ and that $y$ is continuously shift-differentiable in $\bar{u}$. Then $J$ is Fréchet differentiable in a neighborhood $D^{\prime}$ of $\bar{u}$ by the previous arguments. By assumption $u \in D^{\prime} \longmapsto \bar{x}_{k}(u)$ and $u \in D^{\prime} \longmapsto y(u)\left(\bar{x}_{k}(u) \pm\right)$ are continuous at $\bar{u}$. Hence, the operator in the second term of (2.2) with $y, \bar{x}_{k}(u)$ instead of $\bar{y}, \bar{x}_{k}$ acting on $\bar{s}$ is obviously continuous at $\bar{u}$. Moreover, we have for all $\delta y \in L^{r}(I)$ and $u \in D^{\prime}$ with a local Lipschitz constant $L$ of $\phi_{y}$

$$
\left(\phi_{y}\left(y(u), y_{d}\right)-\phi_{y}\left(y(\bar{u}), y_{d}\right), \delta y\right)_{2, I} \leq L\|y(u)-y(\bar{u})\|_{r^{\prime}, I}\|\delta y\|_{r, I} .
$$

The first factor tends to zero by interpolating (2.3) and the $L^{\infty}$-bound. Combining the continuity in $(\delta y, \bar{s}) \in L^{r}(I) \times \mathbb{R}^{K}$ with the continuity of $u \longmapsto T_{s}(u) \in \mathcal{L}\left(U, L^{r}(I) \times \mathbb{R}^{K}\right)$ at $\bar{u}$ now yields the continuity of $d_{u} J(y(u))$ at $\bar{u}$.

Finally, if $y_{d}$ has an approximate discontinuity in $\bar{x}$ and $y_{d}(\bar{x})$ is replaced by $y_{d}(\bar{x}+$ $0 \cdot \operatorname{sgn}(\bar{s}))$ then the directional differentiability can be shown exactly as above by fixing $\delta u$ and taking $\varepsilon \delta u$ instead of $\delta u$. In fact, the only crucial point is the estimate for the resulting expression $I_{1}$. As in (2.7) we obtain $I_{1}=o(\varepsilon)$.

In the following sections we will analyze the control-to-state mapping $u \longmapsto y(t, \cdot ; u)$ implicitly defined by (1.1). As a main result we will show that (1.5) is in general shiftdifferentiable. Then we obtain immediately the differentiability properties of objective functionals (1.2) by using Lemma 2.3.

3. Stability and structure of entropy solutions. As outlined in the introduction our aim is to derive a shift-differentiability result for entropy solutions $y=y(\cdot ; u)$ of

$$
\begin{aligned}
& \left.\partial_{t} y+\partial_{x} f(y)=g\left(t, x, y, u_{1}\right), \quad(t, x) \in \Omega_{T} \stackrel{\text { def }}{=}\right] 0, T[\times \mathbb{R} \\
& y(0, x)=u_{0}(x), \quad x \in \mathbb{R} .
\end{aligned}
$$

with respect to the control $u=\left(u_{0}, u_{1}\right)$, if the initial data $u_{0}$ are variated by a shift-variation and $u_{1}$ by a conventional additive variation. More precisely, let $u_{0} \in P C^{1}\left(\mathbb{R} ; x_{1}, \ldots, x_{N}\right)$, $x_{1}<x_{2}<\ldots<x_{N}$, and $u_{1} \in L^{\infty}\left(0, T ; C^{1}(\mathbb{R})^{m}\right)$ be given and fix some $\left.\left.\bar{t} \in\right] 0, T\right]$. For

$$
W \stackrel{\text { def }}{=} P C^{1}\left(\mathbb{R} ; x_{1}, \ldots, x_{N}\right) \times L^{\infty}\left(0, T ; C^{1}(\mathbb{R})^{m}\right) \times \mathbb{R}^{N}
$$


consider the mapping

$$
\left(w_{0}, w_{1}, \sigma\right) \in W \longmapsto y\left(\bar{t}, \cdot ; u_{0}+S_{u_{0}}^{\left(x_{i}\right)}\left(w_{0}, \sigma\right), u_{1}+w_{1}\right) \in L^{1}([a, b])
$$

for some $a<b$. We will show that this mapping is continuously shift-differentiable in a sufficiently small neighborhood of 0 if at time $\bar{t}$ a nondegeneracy condition is satisfied at the shock locations. We will moreover show in Theorem 11.2 that this nondegeneracy condition holds for almost all $\bar{t}$ if $u_{0} \in P C^{2}\left(\mathbb{R} ; x_{1}, \ldots, x_{N}\right)$ and $u_{1} \in L^{\infty}\left(0, T ; C_{l o c}^{2}(\mathbb{R})^{m}\right)$.

Our analysis is based on the theory of generalized characteristics introduced in [8] to obtain structural information on the solution combined with a duality argument using the adjoint equation to (3.1). This approach has the advantage that we have not to restrict a priori the class of considered entropy solutions. Thus, the results apply to solutions with very complicated structure.

In order to ensure the existence of essentially bounded entropy solutions and to allow the application of the theory of generalized characteristics we make the following Assumptions:

(A2) (A1) holds, $f$ is twice continuously differentiable, $g \in L^{\infty}\left(0, T ; C_{l o c}^{1}\left(\mathbb{R} \times \mathbb{R} \times \mathbb{R}^{m}\right)\right)$ and $g$ is Lipschitz continuous w.r.t. $x$.

(A3) $f^{\prime \prime} \geq m_{f^{\prime \prime}}>0$ for some $m_{f^{\prime \prime}}>0$.

In the next subsection we summarize existence and stability results for entropy solutions of (3.1). In 3.2 we collect from [8] the necessary results on the structure of solutions provided by the theory generalized characteristics. These ingredients will be used in sections 4-9 to prepare the proof of the shift-differentiability result in section 10 .

3.1. Basic properties of entropy solutions. We recall the following existence, uniqueness and stability properties of the state equation (1.1), see e.g. [24] and [20].

THEOREM 3.1. Let (A1) hold. Then for all $u=\left(u_{0}, u_{1}\right) \in L^{\infty}(\mathbb{R}) \times L^{\infty}\left(\Omega_{T}\right)^{m} \stackrel{\text { def }}{=} U$ there exists a unique entropy solution $y=y(u) \in L^{\infty}\left(\Omega_{T}\right)$. After modification on a set of measure zero holds $y \in C\left([0, T] ; L^{1}([-R, R])\right)$ for all $R>0$. Let $M_{u}>0$ and $U_{a d}=$ $\left\{u \in U:\left\|u_{0}\right\|_{\infty} \leq M_{u},\left\|u_{1}\right\|_{\infty} \leq M_{u}\right\}$. Then there are $M_{y}>0$ and $L_{y}>0$ such that for all $u, \hat{u} \in U_{\text {ad }}$ the corresponding solutions $y, \hat{y}$ satisfy

i) $\|y(t, \cdot)\|_{\infty} \leq M_{y}$

ii) $\|y(t, \cdot)-\hat{y}(t, \cdot)\|_{1,[a, b]} \leq L_{y}\left(\left\|u_{0}-\hat{u}_{0}\right\|_{1, I_{t}}+\left\|u_{1}-\hat{u}_{1}\right\|_{1,[0, t] \times I_{t}}\right)$

for all $t \in[0, T], a<b$, where $I_{t}=\left[a-t M_{f^{\prime}}, b+t M_{f^{\prime}}\right]$ with $M_{f^{\prime}}=\max _{|y| \leq M_{y}}\left|f^{\prime}(y)\right|$.

Let moreover (A2), (A3) hold and set $\hat{U}_{a d}=\left\{u \in U_{a d}:\left\|u_{1}\right\|_{L^{\infty}\left(0, T ; C^{1}\right)} \leq M_{u}\right\}$. Then there exists a constant $M_{c r}>0$ such that for all $u \in \hat{U}_{a d}$ and all $\left.\left.t \in\right] 0, T\right]$ with $E=M_{c r} m_{f^{\prime \prime}}$ Oleinik's entropy condition

$$
\partial_{x} y(t, \cdot) \leq \frac{1}{\left(1-e^{-E t}\right) / M_{c r}+e^{-E t} / M}
$$

holds in the sense of distributions, whenever $M \in\left[M_{c r}, \infty\right]$ is such that $\partial_{x} u_{0} \leq M$ in the sense of distributions. In particular, $y(t, \cdot) \in B V_{\text {loc }}(\mathbb{R})$ for all $\left.\left.t \in\right] 0, T\right]$.

Proof. For the first part see e.g. [24]. The Oleinik entropy condition in this form can be deduced by a straightforward extension of the proof in [22] to the inhomogeneous case, see also [20].

Since for all $u \in U_{a d}$ the corresponding solutions $y=y(u)$ of (1.1) satisfy $\|y\|_{\infty} \leq M_{y}$, we may modify $g$ for $|y|>M_{y}$ in such a way that $g$ satisfies instead of the weaker growth condition in (A1) a global Lipschitz condition w.r.t. $y$. To study $y(u)$ for $u \in U_{a d}$ we may therefore assume that instead of (A2) holds

Assumption:

(A2') (A2) is satisfied and $g$ is globally Lipschitz w.r.t $y$. 
By this modification of $g$ we can achieve that also the backward solutions of the characteristic equations associated with (1.1) remain bounded for all end data and not only for end data obtained from bounded forward solutions. Since we will always deal with bounded entropy solutions, we will without restriction assume that (A2') holds.

3.2. Generalized characteristics and the structure of solutions. We assume throughout this section that (A2), (A3) hold and consider controls $u \in \hat{U}_{a d}$ with $\hat{U}_{a d}$ from Theorem 3.1. Hence, we may assume without restriction that also $\left(\mathrm{A} 2^{\prime}\right)$ is satisfied.

Let $u=\left(u_{0}, u_{1}\right) \in \hat{U}_{a d}$ be given. Then (1.1) has by Theorem 3.1 a unique entropy solution $y=y(u) \in L^{\infty}\left(\Omega_{T}\right) \cap C\left([0, T] ; L_{l o c}^{1}(\mathbb{R})\right)$ with $\left.\left.y(t, \cdot) \in B V_{l o c}(\mathbb{R}), t \in\right] 0, T\right]$ and $\|y\|_{\infty} \leq M_{y}$. Hence, $y$ admits left and right limits w.r.t. $x$ for all $\left.\left.t \in\right] 0, T\right]$. Moreover, by (3.2) each discontinuity is admissible, i.e.

$$
y(t, x-) \geq y(t, x+) \quad \text { for all } t \in] 0, T] \text { and all } x \in \mathbb{R} .
$$

These properties allow the application of the theory of generalized characteristics [8]. For notational convenience we consider the representative for $y$ with $y(t, x)=y(t, x-)$. A Lipschitz continuous curve $x=\xi(t)$ defined on $t \in[a, b] \subset[0, T]$ is a (generalized) characteristic if the differential inclusion holds

$$
\dot{\xi}(t) \in\left[f^{\prime}(y(t, \xi(t)+)), f^{\prime}(y(t, \xi(t)-))\right] \quad \text { a.e. on }[a, b] .
$$

The local existence of a characteristic through any $(\bar{t}, \bar{x}) \in \Omega_{T}$ follows from [9], see also [8]. Assumption (A1) ensures that $\|y\|_{\infty} \leq M_{y}$. Thus, characteristics can not escape and exist on the whole interval $[0, T]$. Hence, we can always set $[a, b]=[0, T]$. Since $y$ is a weak solution of (3.1), it can be deduced [8] that actually holds

$$
\dot{\xi}(t)=\left\{\begin{array}{lll}
f^{\prime}(y(t, \xi(t))) & \text { if } y(t, \xi(t)-)=y(t, \xi(t)+) \\
\frac{[f(y(t, \xi(t)))]}{[y(t, \xi(t))]} & \text { if } y(t, \xi(t)-) \neq y(t, \xi(t)+) & \text { a.e. on }[0, \mathrm{~T}] .
\end{array}\right.
$$

A characteristic is called genuine on $[a, b]$, if $y(t, \xi(t)+)=y(t, \xi(t)-)$ for a.a. $t \in[a, b]$.

The study of generalized characteristics in [8] together with the a priori bound $\|y\|_{\infty} \leq M_{y}$ yields the following structure of $y$ :

For each fixed $(\bar{t}, \bar{x}) \in \Omega_{T}$ the one-sided limits $y(\bar{t}, \bar{x} \pm)$ exist and satisfy the entropy condition $y(\bar{t}, \bar{x}-) \geq y(\bar{t}, \bar{x}+)$. Moreover, the minimal and maximal backward characteristics $\xi_{\mp}(t)$ through $(\bar{t}, \bar{x})$ are genuine, i.e. $\left.y\left(t, \xi_{\mp}(t)-\right)=y\left(t, \xi_{\mp}(t)+\right), t \in\right] 0, \bar{t}[$. Moreover, for any genuine characteristic $\xi(t)$ on $[0, \bar{t}]$ holds (with our convention for the choice of $y$ )

$$
\begin{gathered}
\xi(t)=\zeta(t), t \in[0, \bar{t}], \quad y(t, \xi(t))=v(t), t \in] 0, \bar{t}\left[, \quad u_{0}(\xi(0)-) \leq v(0) \leq u_{0}(\xi(0)+)\right. \\
y(\bar{t}, \xi(\bar{t})-) \geq v(\bar{t}) \geq y(\bar{t}, \xi(\bar{t})+)
\end{gathered}
$$

where $(\zeta, v)$ solves the classical characteristic equations

$$
\begin{aligned}
& \dot{\zeta}(t)=f^{\prime}(v(t)) \\
& \dot{v}(t)=g\left(t, \zeta(t), v(t), u_{1}(t, \zeta(t))\right) .
\end{aligned}
$$

In particular, two different genuine characteristics may intersect only at their end points. Finally, if $\xi$ is the minimal characteristic $\xi_{-}$or the maximal characteristic $\xi_{+}$through $(\bar{t}, \bar{x})$ then (3.3) holds with the solution $(\zeta, v)$ of (3.4) for the initial values

$$
(\zeta(\bar{t}), v(\bar{t}))= \begin{cases}(\bar{x}, y(\bar{t}, \bar{x}-)) & \text { if } \xi=\xi_{-} \\ (\bar{x}, y(\bar{t}, \bar{x}+)) & \text { if } \xi=\xi_{+}\end{cases}
$$


We remark that for any minimal or maximal backward characteristic $\xi_{\mp}(t)$ the point $z=\xi_{\mp}(0)$ is a continuity point of $u_{0}$ or a non entropy-admissible discontinuity, i.e. $u_{0}(z-)<u_{0}(z+)$.

Denote for $u_{1} \in L^{\infty}\left(0, T ; C^{1}(\mathbb{R})^{m}\right)$ and $z, w \in \mathbb{R}$ by $\left(\zeta\left(\cdot ; z, w, u_{1}\right), v\left(\cdot ; z, w, u_{1}\right)\right)$ the solution of (3.4) with

$$
\zeta\left(0 ; z, w, u_{1}\right)=z, \quad v\left(0 ; z, w, u_{1}\right)=w .
$$

Let $(\bar{t}, \bar{x}) \in \Omega_{T}$ be a point of continuity of $y$ w.r.t. $x$. Then $y$ is by [8] continuous at $(\bar{t}, \bar{x})$ and the backward characteristic $\xi$ through $(\bar{t}, \bar{x})$ is unique and genuine. Moreover, $\bar{z}=\xi(0)$ is a continuity point of $u_{0}$ or $u_{0}(\bar{z}-)<u_{0}(\bar{z}+)$. In the first case we have

$$
\begin{aligned}
\bar{x} & =\zeta\left(\bar{t} ; \bar{z}, u_{0}(\bar{z}), u_{1}\right) \\
y(\bar{t}, \bar{x}) & =v\left(\bar{t} ; \bar{z}, u_{0}(\bar{z}), u_{1}\right) .
\end{aligned}
$$

In the second case $(\bar{t}, \bar{x})$ lies on a rarefaction wave, i.e.

$$
\begin{aligned}
\bar{x} & =\zeta\left(\bar{t} ; \bar{z}, \bar{w}, u_{1}\right) \\
y(\bar{t}, \bar{x}) & =v\left(\bar{t} ; \bar{z}, \bar{w}, u_{1}\right)
\end{aligned}
$$

with some $\bar{w} \in\left[u_{0}(\bar{z}-), u_{0}(\bar{z}+)\right]$.

To study the smoothness of $(t, x, \hat{u}) \longmapsto y(t, x ; \hat{u})$ in a suitable neighborhood of $(\bar{t}, \bar{x}, u)$ we will show that (3.7) can locally be solved for $\bar{z}$ (or (3.9) for $\bar{w}$ ) as long as $(\bar{t}, \bar{x})$ is not a shock generation point yielding with (3.8) (or (3.10)) an expression for $y$.

We begin by stating smoothness properties of the functions on the right hand side in (3.9) and (3.10). The following result on ordinary differential equations will be useful.

Proposition 3.2. Let $h(t, X, U) \in L^{\infty}\left(0, T ; C_{\text {loc }}^{1}\left(\mathbb{R}^{n} \times \mathbb{R}^{m}\right)^{n}\right)$ and Lipschitz w.r.t. $X$. Set $\bar{H} \stackrel{\text { def }}{=}\left\{(Z, U) \in \mathbb{R}^{n} \times L^{\infty}\left(0, T ; C^{1}\left(\mathbb{R}^{n}\right)^{m}\right):\|U\|_{L^{\infty}\left(0, T ; C^{1}\left(\mathbb{R}^{n}\right)\right)}<M\right\}$ for some $M>0$. Then for all $(Z, U) \in \bar{H}$ there exists a unique solution $X=X(\cdot ; Z, U) \in$ $C^{0,1}([0, T])^{n}$ of

$$
\dot{X}(t)=h(t, X(t), U(t, X(t))), \quad X(0)=Z
$$

and the mapping $(Z, U) \in\left(\bar{H},\|\cdot\|_{\mathbb{R}^{n} \times L^{2}\left(0, T ; C^{i}\left(\mathbb{R}^{n}\right)^{m}\right)}\right) \longmapsto X(\cdot ; Z, U) \in C([0, T])^{n}$ is Lipschitz continuous for $i=0$ and continuously Fréchet differentiable for $i=1 .(t, Z) \longmapsto$ $X(t ; Z, U)$ is Lipschitz on $[0, T] \times \mathbb{R}^{n}$ for $U \in \bar{H}$. Finally, for any compact set $S \subset \mathbb{R}^{n}$ the mapping $U \in L^{\infty}\left(0, T ; C^{1}\left(\mathbb{R}^{n}\right)^{m}\right) \longmapsto X(\cdot ; \cdot, U) \in C([0, T] \times S)^{n}$ is continuously Fréchet differentiable.

The proof is standard and can e.g. be obtained by a refinement of the analysis in the Appendix of [21] using the fact that the Nemyckii operator

$$
(Z, U) \in L^{\infty}([0, T]) \times\left(L^{\infty}\left(0, T ; C^{1}(\mathbb{R})\right),\|\cdot\|_{L^{2}\left(0, T ; C^{1}(\mathbb{R})\right)}\right) \longmapsto U(\cdot, Z(\cdot)) \in L^{2}([0, T])
$$

is continuously Fréchet differentiable. Since the remainder term in the first order expansion of $(Z, U) \longmapsto X(\cdot ; Z, U)$ can be estimated uniformly for all $Z$ in a compact set, the last assertion follows immediately. We omit the technical details.

Now we obtain the following properties for solutions of (3.4).

LEMMA 3.3. Let (A2) hold and denote for $\left(z, w, u_{1}\right) \in \mathbb{R} \times \mathbb{R} \times L^{\infty}\left(0, T ; C^{1}(\mathbb{R})^{m}\right)$ by $(\zeta, v)\left(\cdot ; z, w, u_{1}\right)$ the solution of (3.4) for initial data (3.6). Let $M_{w}, M_{u}>0$ be given and

$$
\begin{gathered}
H_{i} \stackrel{\text { def }}{=} \mathbb{R}^{2} \times L^{2}\left(0, T ; C^{i}(\mathbb{R})^{m}\right), i=0,1, \\
H \stackrel{\text { def }}{=}\left\{\left(z, w, u_{1}\right) \in H_{1}:|w|<M_{w},\left\|u_{1}\right\|_{L^{\infty}\left(0, T ; C^{1}(\mathbb{R})\right)}<M_{u}\right\} .
\end{gathered}
$$


Then the mapping

$$
\left(z, w, u_{1}\right) \in\left(H,\|\cdot\|_{H_{i}}\right) \longmapsto(\zeta, v)\left(\cdot ; z, w, u_{1}\right) \in C([0, T])^{2}
$$

is Lipschitz continuous for $i=0$ and continuously Fréchet differentiable for $i=1$ and on $H$ the right hand side is uniformly Lipschitz w.r.t. t. Moreover, with $\delta \nu=\left(\delta z, \delta w, \delta u_{1}\right)$ one has

$$
d_{\left(z, w, u_{1}\right)}(\zeta, v)\left(\cdot ; z, w, u_{1}\right) \cdot \delta \nu=(\delta \zeta, \delta v)\left(\cdot ; z, w, u_{1} ; \delta \nu\right)
$$

where $(\delta \zeta, \delta v)=(\delta \zeta, \delta v)\left(\cdot ; z, w, u_{1} ; \delta z, \delta w, \delta u_{1}\right)$ solves the linearized equation

$$
\begin{aligned}
& \dot{\delta \zeta}=f^{\prime \prime}(v) \delta v \\
& \dot{\delta v}=g_{x}(:) \delta \zeta+g_{y}(:) \delta v+g_{u_{1}}(:)\left(\partial_{x} u_{1}(t, \zeta) \delta \zeta+\delta u_{1}(t, \zeta)\right) \\
& \delta \zeta(0)=\delta z, \quad \delta v(0)=\delta w,
\end{aligned}
$$

with $(:)=\left(t, \zeta, v, u_{1}(t, \zeta)\right)$. The Fréchet derivative (3.12) can be continuously extended to $\mathcal{L}\left(H_{0}, C([0, T])\right)$ uniformly bounded on bounded subsets of $H$. Finally, for any closed $S \subset \Omega_{T}^{c l}$ and any bounded interval $J$ the mapping

$$
\left(z, u_{0}, u_{1}\right) \in C(S ; J) \times C^{1}(J) \times L^{\infty}\left(0, T ; C^{1}(\mathbb{R})^{m}\right) \longmapsto(\zeta, v)\left({ }_{t} ; z, u_{0}(z), u_{1}\right) \in C(S)^{2}
$$

is continuously Fréchet differentiable where ${ }_{t}$ denotes the projection $(t, x) \longmapsto t$. If $\left(\mathrm{A} 2^{\prime}\right)$ holds then the same statements are true for backward solutions of (3.4).

Proof. We can apply Proposition 3.2 if an a priori bound for $v$ in (3.4) is known, since this ensures with (A2) that the right hand side in (3.4) admits a Lipschitz constant w.r.t. $\left(\zeta, v, u_{1}\right)$ for all $\left(\zeta, v, u_{1}\right)$ of interest. To derive such a bound we use (A1) and get constants $C_{1}, C_{2}>0$ with $\left.\frac{d}{d t}|v(t)| \leq C_{1}+C_{2}|v(t)|, t \in\right] 0, T\left[\right.$. Since $|v(0)|=|w| \leq M_{w}$, the Gronwall Lemma yields

$$
|v(t)| \leq\left(M_{w}+C_{1} T\right) e^{C_{2} T}, \quad t \in[0, T] .
$$

Now the proof can be obtained by using Proposition 3.2.

The fact that (3.12) can be continuously extended to $\mathcal{L}\left(H_{1}, C([0, T])\right)$ is obvious by the properties of (3.13). The differentiability of (3.14) follows from Proposition 3.2, since the Nemyckii operator

$$
\left(z, u_{0}\right) \in C(S ; J) \times C^{1}(J) \longmapsto u_{0}(z(\cdot)) \in C(S)
$$

is continuously Fréchet differentiable.

If $\left(\mathrm{A} 2^{\prime}\right)$ is satisfied then we have the stronger growth estimate $\left|g\left(t, x, y, u_{1}\right)\right| \leq C_{1}+C_{2}|y|$ and the above arguments can be applied to backward solutions.

To ensure backward stability of solutions to (3.4) for all end data - not only the relevant ones obtained from forward solutions - it will be convenient to assume (A2') instead of (A2) which may be done without restriction by our considerations at the end of section 3.1.

For the further analysis of the structure of entropy solutions we assume that $u_{0} \in$ $P C^{1}\left(\mathbb{R} ; x_{1}, \ldots, x_{N}\right), x_{1}<\ldots<x_{N}$, and $u_{1} \in L^{\infty}\left(0, T ; C^{1}(\mathbb{R})^{m}\right)$.

3.3. Classification of continuity points. For further reference we distinguish several cases for continuity points $(\bar{t}, \bar{x})$. We denote the genuine backward characteristic through $(\bar{t}, \bar{x})$ by $\xi$ and set $\bar{z}=\xi(0)$ :

Case C: Let $(\bar{t}, \bar{x})$ be a continuity point of $y=y(u)$ such that $\bar{z} \neq x_{i}, i=1, \ldots, N$. Since $u_{0} \in P C^{1}\left(\mathbb{R} ; x_{1}, \ldots, x_{N}\right)$, there is an interval $J$ containing $\bar{z}$ such that $\left.u_{0}\right|_{J} \in C^{1}(J)$. Now

$$
\left(z, u_{0}, u_{1}\right) \in J \times C^{1}(J) \times L^{\infty}\left(0, T ; C^{1}(\mathbb{R})^{m}\right) \longmapsto(\zeta, v)\left(\cdot ; z, u_{0}(z), u_{1}\right) \in C([0, T])^{2}
$$


is continuously Fréchet differentiable by Lemma 3.3. Hence, $\frac{d}{d z} \zeta\left(t ; z, u_{0}(z), u_{1}\right)$ exists and is continuous on $(t, z) \in[0, T] \times J$. Since genuine characteristics may intersect only at their end points and contain only continuity points, it is obvious that [8]

$$
\left.\frac{d}{d z} \zeta\left(t ; z, u_{0}(z), u_{1}\right)\right|_{z=\bar{z}} \geq 0,0 \leq t \leq \bar{t} .
$$

Moreover, if $(\bar{t}, \bar{x})$ is not an element of the shock set, i.e. if the unique forward characteristic is genuine until some $\bar{t}+\tau, \tau>0$, then there is $\beta>0$ with

$$
\left.\frac{d}{d z} \zeta\left(t ; z, u_{0}(z), u_{1}\right)\right|_{z=\bar{z}} \geq \beta>0,0 \leq t \leq \bar{t} .
$$

In fact, we have

$$
\left.\frac{d}{d z} \zeta\left(t ; z, u_{0}(z), u_{1}\right)\right|_{z=\bar{z}}=\delta \zeta\left(t ; \bar{z}, u_{0}(\bar{z}), u_{1} ; 1, u_{0}^{\prime}(\bar{z}), 0\right)
$$

with $\delta \zeta$ given by (3.13). Moreover, all points on the genuine backward characteristic $\zeta(t)=$ $\zeta\left(t ; \bar{z}, u_{0}(\bar{z}), u_{1}\right)$ are continuity points. Assume that for the right hand side of (3.18) holds $\delta \zeta(\tilde{t})=0$ at some $\tilde{t} \in[0, \tilde{t}]$. Then $\delta \dot{\zeta}(\tilde{t}) \neq 0$, since otherwise by the first line in (3.13) $\delta v(\tilde{t})=0$. This is impossible because the unique backward solution of (3.13) would vanish in contradiction to the initial values. From (3.16) we thus have $\delta \dot{\zeta}(\tilde{t})<0$ and therefore $\left.\frac{d}{d z} \zeta\left(t ; z, u_{0}(z), u_{1}\right)\right|_{z=\bar{z}}<0$ for small $t>\tilde{t}$. Hence, the unique forward characteristic through $(\tilde{t}, \zeta(\tilde{t}))$ can not be genuine, since the unique candidate $\zeta(t)=\zeta\left(t ; \bar{z}, u_{0}(\bar{z}), u_{1}\right)$ is not admissible by (3.16). Since the left hand side of (3.17) is continuous on $[0, \bar{t}],(3.17)$ must hold for some $\beta>0$.

Case CB: If $(\bar{t}, \bar{x})$ is a continuity point, $\bar{z}=x_{i}$ and $u_{0}$ is continuous at $\bar{z}$ then by the same arguments the one-sided derivatives must satisfy (3.16) and in addition (3.17) if $(\bar{t}, \bar{x})$ is not in the shock set.

Cases R, RB: If $(\bar{t}, \bar{x})$ is a continuity point and $\bar{z}=x_{i}$ with $u_{0}(\bar{z}-)<u_{0}(\bar{z}+)$ then three cases can occur.

Case R: If all backward characteristics through $(\bar{t}, x)$ with $x$ in a small neighborhood of $\bar{x}$ meet $t=0$ in $\bar{z}$ then similar arguments as above show that with $\bar{w}$ from (3.9) holds

$$
\left.\frac{d}{d w} \zeta\left(t ; \bar{z}, w, u_{1}\right)\right|_{w=\bar{w}} \geq 0,0 \leq t \leq \bar{t} .
$$

and if $(\bar{t}, x)$ is not a point of the shock set there is $\beta>0$ with

$$
\left.\frac{d}{d w} \zeta\left(t ; \bar{z}, w, u_{1}\right)\right|_{w=\bar{w}} \geq \beta t>0, \quad 0<t \leq \bar{t}
$$

Case RB: Otherwise, $(\bar{t}, \bar{x})$ lies on the left or right boundary of a rarefaction wave and the one-sided derivatives satisfy (3.16) and (3.19) (and moreover (3.17) and (3.20) if $(\bar{t}, x)$ is no shock generation point), respectively.

Notation. The instance that $(\bar{t}, \bar{x})$ is no shock generation point and satisfies case $\mathrm{C}, \mathrm{CB}, \mathrm{R}$ or $\mathrm{RB}$, respectively, is denoted by $\mathrm{C}^{c}, \mathrm{CB}^{c}, \mathrm{R}^{c}$ or $\mathrm{RB}^{c}$, respectively.

3.4. Classification of shock points. Let now $(\bar{t}, \bar{x})$ be a shock point located on the shock curve $\eta(t)$. We know from [8] and 3.2 that the minimal and maximal backward characteristics $\xi_{\mp}(t)$ through $(\bar{t}, \bar{x})$ are genuine with initial condition (3.5). Thus, if we set $\bar{z}^{\mp}=\xi_{\mp}(0)$ we can classify the left and right states of the shock exactly as continuity points before depending on whether $\bar{z}^{\mp}=x_{i}$ for some $i$ or not. Moreover, the corresponding equations (3.7), (3.8) or (3.9), (3.10) hold with $\bar{z}^{\mp}$ and $y(\bar{t}, \bar{x} \mp)$ instead of $\bar{z}, y(\bar{t}, \bar{x})$.

Notation. We call for example the shock point $(\bar{t}, \bar{x})$ of class $\mathrm{C}^{c} \mathbf{R}^{c}$ if the left state satisfies case $\mathrm{C}^{c}$ and the right state case $\mathrm{R}^{c}$. 
4. Differentiability at continuity points. We start by analyzing the differentiability properties at continuity points that are no shock generation points and that are moreover not located on the boundary of a rarefaction wave (case $\mathrm{C}^{c}$ or $\mathrm{R}^{c}$ ).

4.1. Differentiability in continuity points of class $\mathbf{C}^{c}$. We study first the case $\mathbf{C}^{c}$, i.e. $(\bar{t}, \bar{x})$ satisfies case $\mathrm{C}$ and is no shock generation point. By continuity there are $z_{-}<\bar{z}<z_{+}$ such that

$$
\left.u_{0} \in C^{1}(J), \frac{d}{d z} \zeta\left(t ; z, u_{0}(z), u_{1}\right) \geq \beta>0 \quad \forall t \in[0, \bar{t}], \forall z \in\right] z_{-}-\rho, z_{+}+\rho[\stackrel{\text { def }}{=} J
$$

for some $\beta, \rho>0$. This allows us to solve (3.7) locally for $\bar{z}$ yielding with (3.8) a local regularity result for $y$.

4.1.1. Solution of the characteristic equations. We have the following result on the solvability of (3.7).

LEMMA 4.1. Let $u_{0} \in P C^{1}\left(\mathbb{R} ; x_{1}, \ldots, x_{N}\right), u_{1} \in L^{\infty}\left(0, T ; C^{1}(\mathbb{R})^{m}\right)$, and let (4.1) hold for some $\beta, \rho>0$. Then there is $\tau>0$ and a neighborhood $V \subset C^{1}(J) \times$ $L^{\infty}\left(0, T ; C^{1}(\mathbb{R})^{m}\right)$ of $u=\left(u_{0}, u_{1}\right)$ such that

$$
\frac{d}{d z} \zeta\left(t ; z, \hat{u}_{0}(z), \hat{u}_{1}\right) \geq \frac{\beta}{2}>0 \quad \forall(t, z) \in[0, \bar{t}+\tau] \times J, \quad \forall \hat{u} \in V .
$$

Moreover, for all $\hat{u} \in V$ and all $(t, x)$ in the stripe

$$
S=S(\tau) \stackrel{\text { def }}{=}\left\{(t, x): t \in[0, \bar{t}+\tau], x \in\left[\xi_{-}(t), \xi_{+}(t)\right]\right\}, \quad \xi_{\mp}(t)=\zeta\left(t ; z_{\mp}, u_{0}\left(z_{\mp}\right), u_{1}\right)
$$

the equation

$$
x=\zeta\left(t ; z, \hat{u}_{0}(z), \hat{u}_{1}\right)
$$

has in $J$ a unique solution $z=Z(t, x, \hat{u})$. Set

$$
Y(t, x, \hat{u}) \stackrel{\text { def }}{=} v\left(t ; Z(t, x, \hat{u}), \hat{u}_{0}(Z(t, x, \hat{u})), \hat{u}_{1}\right)
$$

Then $Z(\cdot, \hat{u}), Y(\cdot, \hat{u}) \in C^{0,1}(S)$. The mappings

$$
\begin{aligned}
&(x, \hat{u}) \in] \xi_{-}(t), \xi_{+}(t)[\times V \longmapsto(Z, Y)(t, x, \hat{u}), \quad t \in[0, \bar{t}+\tau[ \\
& \hat{u} \in V \longmapsto(Z, Y)(\cdot, \hat{u}) \in C(S)^{2}
\end{aligned}
$$

are continuously Fréchet differentiable. The derivatives of (4.5) are

$$
\begin{gathered}
d_{(x, u)} Z(t, x, \hat{u}) \cdot(\delta x, \delta u)=\frac{\delta x-\delta \zeta\left(t ; z, \hat{u}_{0}(z), \hat{u}_{1} ; 0, \delta u_{0}(z), \delta u_{1}\right)}{\delta \zeta\left(t ; z, \hat{u}_{0}(z), \hat{u}_{1} ; 1, \hat{u}_{0}^{\prime}(z), 0\right)} \\
d_{(x, u)} Y(t, x, \hat{u}) \cdot(\delta x, \delta u)= \\
\quad \delta v\left(t ; z, \hat{u}_{0}(z), \hat{u}_{1} ; 1, \hat{u}_{0}^{\prime}(z), 0\right) d_{(x, u)} Z(t, x, \hat{u}) \cdot(\delta x, \delta u) \\
+\delta v\left(t ; z, \hat{u}_{0}(z), \hat{u}_{1} ; 0, \delta u_{0}(z), \delta u_{1}\right)
\end{gathered}
$$

where $z=Z(t, x, \hat{u})$ and $(\delta \zeta, \delta v)$ are given by (3.13). The derivative of (4.6) is

$$
d_{u}(Z, Y)(\cdot, \hat{u}) \cdot \delta u=d_{(x, u)}(Z, Y)(\cdot, \hat{u}) \cdot(0, \delta u) .
$$

REMARK 4.2. By construction, $Y(\cdot, \hat{u}) \in C^{0,1}(S)$ is on $S$ classical solution of (1.1) for the control $\hat{u} \in V$. 
REMARK 4.3. It is not difficult to show that $\delta Y=d_{u} Y(\cdot, u) \cdot \delta u \in C(S)$ is the unique broad solution (i.e. solution along characteristics) of the linearized equation

$$
\begin{aligned}
& \partial_{t} \delta Y+\partial_{x}\left(f^{\prime}(Y) \delta Y\right)=g_{y}\left(t, x, Y, u_{1}\right) \delta Y+g_{u_{1}}\left(t, x, Y, u_{1}\right) \delta u_{1}, \quad(t, x) \in S \\
& \delta Y(0, x)=\delta u_{0}(x), \quad x \in\left[z_{-}, z_{+}\right]
\end{aligned}
$$

where $Y=Y(\cdot, u)$. By Remark 4.2 and the differentiability of (4.6) we see from

$$
\partial_{t} Y(\cdot ; \hat{u})+\partial_{x} f(Y(\cdot ; \hat{u}))=g\left(t, x, Y(\cdot ; \hat{u}), \hat{u}_{1}\right)
$$

with $\hat{u}=u+\sigma \delta u$ by applying test functions $p \in C^{1}(S)$, integrating by parts and taking the derivative in $\sigma=0$ that $\delta Y$ is also a weak solution of (4.10). Even more, for any domain $D \subset S$ with Lipschitz boundary and any $p \in C^{0,1}(D)$ holds

$$
\begin{aligned}
& \left(p\left(n_{1}+n_{2} f^{\prime}(Y)\right), \delta Y\right)_{2, \partial D}= \\
& =\left(\partial_{t} p+f^{\prime}(Y) \partial_{x} p+g_{y}\left(t, x, Y, u_{1}\right) p, \delta Y\right)_{2, D}+\left(p g_{u_{1}}\left(t, x, Y, u_{1}\right), \delta u_{1}\right)_{2, D}
\end{aligned}
$$

where $\left(n_{1}, n_{2}\right)^{T}$ is the unit outer normal of $D$. In section 11 we will choose $p$ as solution of the adjoint equation (1.6) to obtain a gradient representation of (1.2).

Notation. Given $\bar{t}, z_{-}, z_{+}$satisfying (4.1) it will be convenient to indicate by

$$
(Y, Z, V, S(\tau), J)=\mathrm{YC}\left(u, \bar{t},\left[z_{-}, z_{+}\right]\right)
$$

that the open interval $J \supset\left[z_{-}, z_{+}\right]$, the stripe $S=S(\tau)$, the neighborhood $V$ and the functions $Y, Z$ are obtained by applying Lemma 4.1 .

Proof. (of Lemma 4.1) Let $\left(\hat{u}_{0}, \hat{u}_{1}\right) \in C^{1}(J) \times L^{\infty}\left(0, T ; C^{1}(\mathbb{R})^{m}\right)$. We have already observed that Lemma 3.3 implies the continuous Fréchet differentiability of (3.15). Thus, we deduce from (4.1) by continuity that (4.2) holds with a sufficiently small neighborhood $V \subset C^{1}(J) \times L^{\infty}\left(0, T ; C^{1}(\mathbb{R})^{m}\right)$ of $u$ and $\tau>0$ small enough. Hence, for all $t \in[0, \bar{t}+\tau]$ and $\hat{u} \in V$ the mapping

$$
z \in J \longmapsto \zeta\left(t ; z, \hat{u}_{0}(z), \hat{u}_{1}\right)
$$

is strictly monotone increasing and $] \zeta\left(t ; z_{-}, \hat{u}_{0}\left(z_{-}\right), \hat{u}_{1}\right)-\beta \rho / 2, \zeta\left(t ; z_{+}, \hat{u}_{0}\left(z_{+}\right), \hat{u}_{1}\right)+\beta \rho / 2[$ is contained in its image. Hence, for sufficiently small $V$ and $\tau$ we get by continuity that $\left[\xi_{-}(t), \xi_{+}(t)\right]$ is contained in the image for all $t \in[0, \bar{t}+\tau]$ and all $\hat{u} \in V$. As a consequence, for all $(t, x) \in S$ and all $\hat{u} \in V$ there exists exactly one solution $z=Z(t, x, \hat{u}) \in J$ of (4.3). Since $Z(t, x, \hat{u}) \in J$ for all considered $x, \hat{u}$, we conclude from (4.2), (4.3) and the continuous Fréchet differentiability of (3.15) by the implicit function theorem that the first component of (4.5) is continuously Fréchet differentiable. By (4.4) and the continuous differentiability of (3.15) the second component in (4.5) is also continuously Fréchet differentiable. The formula (4.7) is an immediate consequence of the implicit function theorem and (4.8) follows from (4.4). The Lipschitz continuity of $Z(\cdot, \hat{u})$ follows directly from (4.2) and the Lipschitz continuity of (4.3) w.r.t $t, x$. Now the Lipschitz continuity of $Y(\cdot, \hat{u})$ is clear by (4.4) and the Lipschitz continuity of $v\left(t ; z, w, \hat{u}_{1}\right)$ w.r.t. $t, z, w$.

To show the differentiability of (4.6) we observe that for all $\hat{u} \in V$ the function $Z(\cdot, \hat{u})$ is in $C(S ; J)$ and satisfies $F(Z(\cdot, \hat{u}), \hat{u})=0$ with the operator

$$
\begin{aligned}
F:\left(z, \hat{u}_{0}, \hat{u}_{1}\right) & \in C(S ; J) \times C^{1}(J) \times L^{\infty}\left(0, T ; C^{1}(\mathbb{R})^{m}\right) \\
& \longmapsto\left(\zeta\left({ }_{t} ; z(\cdot), \hat{u}_{0}(z(\cdot)), \hat{u}_{1}\right)-\cdot_{x}\right) \in C(S),
\end{aligned}
$$


where ${ }^{t},{ }^{\cdot} x$ denote the projections on the $t$ - and $x$-component, respectively. $F$ is continuously Fréchet differentiable by Lemma 3.3 and we have obviously

$$
d_{z} F(z, \hat{u}) \cdot \delta z=\left(\left.(t, x) \longmapsto \frac{d}{d \tilde{z}} \zeta\left(t ; \tilde{z}, \hat{u}_{0}(\tilde{z}), \hat{u}_{1}\right)\right|_{\tilde{z}=z(t, x)} \delta z(t, x)\right) .
$$

By (4.2) we get that $\left(d_{z} F(z, \hat{u})\right)^{-1}$ exists and is bounded for all $z \in C(S ; J)$ and all $\hat{u} \in V$. Hence, the first component of (4.6) is continuously Fréchet differentiable by the implicit function theorem, now by (4.4) and Lemma 3.3 also the second. The formula (4.9) is obvious. The properties of these mappings follow directly from Lemma 3.3.

4.1.2. A differentiability result for case $\mathbf{C}^{c}$. We are now able to characterize the properties of $y$ in continuity points of class $\mathrm{C}^{c}$.

LEMmA 4.4. Let $u=\left(u_{0}, u_{1}\right) \in P C^{1}\left(\mathbb{R} ; x_{1}, \ldots, x_{N}\right) \times L^{\infty}\left(0, T ; C^{1}(\mathbb{R})^{m}\right)$. Let $(\bar{t}, \bar{x}) \in \Omega_{T}$ be a point of continuity of $y=y(\cdot ; u)$ outside the shock set such that $\bar{z} \neq x_{i}$, $1 \leq i \leq N$, holds for $\bar{z}$ given by (3.7). Then the following statements are true:

i) There is a maximal nonempty open interval I such that $\{\bar{t}\} \times I$ does not contain points of the shock set and that all backward characteristics through $(\bar{t}, x), x \in I$, meet $t=0$ not in $x_{i}, i=1, \ldots, N . y(\bar{t}, \cdot ; u)$ is continuously differentiable on $I$.

ii) Let $\hat{I}=] x_{-}, x_{+}\left[\right.$be an arbitrary interval with closure in $I$, denote by $\xi_{\mp}$ the genuine backward characteristics through $\left(\bar{t}, x_{\mp}\right)$ and set $z_{\mp}=\xi_{\mp}(0)$. Then there are $\beta>0$, $\rho>0$ such that (4.1) is satisfied. Let $(Y, Z, V, S(\tau), J)=\mathrm{YC}\left(u, \bar{t},\left[z_{-}, z_{+}\right]\right)$be obtained according to Lemma 4.1. Given $M_{\infty}>0$ there are $R>0, \nu>0$ such that after a possible reduction of $\tau$ and $V$ holds:

$$
\begin{gathered}
y(t, x ; \hat{u})=Y\left(t, x,\left.\hat{u}_{0}\right|_{J}, \hat{u}_{1}\right) \quad \forall(t, x) \in S, \quad \forall \hat{u} \in \hat{V}, \text { where } \\
\hat{V} \stackrel{\text { def }}{=}\left\{\left(\hat{u}_{0}, \hat{u}_{1}\right) \in L^{\infty}(\mathbb{R}) \times L^{\infty}\left(0, T ; C^{1}(\mathbb{R})^{m}\right):\left(\left.\hat{u}_{0}\right|_{J}, \hat{u}_{1}\right) \in V,\right. \\
\left.\left\|\hat{u}_{0}-u_{0}\right\|_{\infty, \mathbb{R} \backslash J} \leq M_{\infty},\left\|\hat{u}_{0}-u_{0}\right\|_{1,[-R, R] \backslash J} \leq \nu\right\} .
\end{gathered}
$$

Hence, the differentiability results of Lemma 4.1 for $Y$ carry over to $y$.

Proof. i): $(\bar{t}, \bar{x})$ is of class $\mathrm{C}^{c}$, since $\bar{z} \neq x_{i}, i=1, \ldots, N$, and $(\bar{t}, \bar{x})$ is a continuity point outside the shock set. Hence, we know that (3.17) holds for some $\beta>0$ and we find $z_{-}<\bar{z}<z_{+}, \rho>0$ such that (4.1) is satisfied. Therefore, Lemma 4.1 is applicable yielding $(Y, Z, V, S(\tau), J)=\mathrm{YC}\left(u, \bar{t},\left[z_{-}, z_{+}\right]\right)$. Then clearly $u_{0} \in C^{1}(J), i=1, \ldots, N$. By the above mentioned results of [8] we have, cf. (3.7), (3.8),

$$
y(\bar{t}, \bar{x} ; u)=Y(\bar{t}, \bar{x}, u) .
$$

Now $x \longmapsto y(\bar{t}, x ; u)$ is continuous outside a countable set and the identities (3.7), (3.8) with $\tilde{x}, \tilde{z}$ instead of $\bar{x}, \bar{z}$ hold for all continuity points $(\bar{t}, \tilde{x})$. Using the backward stability of (3.4) according to Lemma 3.3 we see that $\tilde{z} \rightarrow \bar{z}$ for continuity points $\tilde{x}$ with $\tilde{x} \rightarrow \bar{x}$. Hence, for all continuity points $\tilde{x} \in] \xi_{-}(\bar{t}), \xi_{+}(\bar{t})[$ with $\tilde{z} \in J$ we must have $\tilde{z}=Z(\bar{t}, \tilde{x}, u)$ and thus

$$
y(\bar{t}, x ; u)=Y(\bar{t}, x, u)
$$

for all $x \in \bar{I} \stackrel{\text { def }}{=}] \bar{x}_{-}, \bar{x}_{+}[$, where $\bar{I}$ is sufficiently small with $\bar{x} \in \bar{I}, \bar{I} \subset] \xi_{-}(\bar{t}), \xi_{+}(\bar{t})[$ (first for the dense set of continuity points $x=\tilde{x} \in \bar{I}$ and thus for all $x \in \bar{I}$ by our convention for the choice of $y$ ). This shows that $y(\bar{t}, \cdot ; u)$ is continuously differentiable on $\bar{I} \neq \emptyset$. Moreover $\{\bar{t}\} \times \bar{I}$ does not contain points of the shock set, since (4.1) holds, and all backward characteristics starting in $\{\bar{t}\} \times \bar{I}$ meet $t=0$ not in $x_{i}, i=1, \ldots, N$. Hence, there exists a maximal open nonempty interval $I$ with the asserted properties.

ii): Let $\hat{I} \stackrel{\text { def }}{=}] x_{-}, x_{+}\left[\neq \emptyset\right.$ be arbitrary with closure in $I$. Now for any point $\bar{x} \in\left[x_{-}, x_{+}\right]$ we can argue as above and find an interval-neighborhood $\bar{I}$ with the above properties. Taking 
a finite covering we get the following: Denote by $\xi_{\mp}$ the genuine backward characteristics through $\left(\bar{t}, x_{\mp}\right)$, set $z_{\mp}=\xi_{\mp}(0)$. By the finite covering we obtain $\rho>0$ and $\beta>0$ such that (4.1) holds. Hence, Lemma 4.1 yields $(Y, Z, V, S(\tau), J)=\mathrm{YC}\left(u, \bar{t},\left[z_{-}, z_{+}\right]\right)$. Then we have from the proof of i) that

$$
y(\bar{t}, x ; u)=Y(\bar{t}, x, u)
$$

for all $x \in \hat{I}$. We show, that also $y(\cdot ; u)=Y(\cdot, u)$ on $S=S(\tau)$ after a possible reduction of $\tau>0$. In fact, $\left(\bar{t}, x_{\mp}\right)$ are continuity points of $y(\cdot ; u)$, since $y(\bar{t}, \cdot ; u)$ is continuous at $x_{\mp}$, cf. [8]. Hence, $\left(\bar{t}, x_{\mp}\right)$ are by (4.1) and [8, Lem. 5.2] no shock generation points, i.e. the genuine characteristics $\xi_{\mp}$ remain genuine until $\bar{t}+\tau$ after a possible reduction of $\tau>0$. Now take any $(t, x) \in S$. The extreme backward characteristics through $(t, x)$ are genuine and may not intersect $\xi_{\mp}$. Hence, they stay in $S$ and must thus coincide with $\zeta\left(\cdot ; Z(t, x, u), u_{0}(Z(t, x, u)), u_{1}\right)$ which yields

$$
y(t, x ; u)=Y(t, x, u) \quad \forall(t, x) \in S .
$$

Since $\hat{I} \stackrel{\text { def }}{=}] x_{-}, x_{+}[$was an arbitrary interval with closure in $I$, the same arguments apply to $\tilde{I}=] x_{-}-3 \eta, x_{+}+3 \eta[$ for $\eta>0$ small enough yielding after a possible reduction of $\tau$

$$
y(t, x ; u)=Y(t, x, u) \quad \forall(t, x) \in \tilde{S}, \quad Z(t, x, u) \in J \quad \forall(t, x) \in \tilde{S} .
$$

where $\tilde{S}=\tilde{S}(\tau)$ is confined by the genuine backward characteristics $\tilde{\xi}_{\mp}$ through $\left(\bar{t}, x_{\mp} \mp 3 \eta\right)$. We can clearly choose $\tau>0$ small enough such that with $\tilde{t}=\bar{t}+\tau$ holds $\tilde{\xi}_{-}(\tilde{t})<\xi_{-}(\tilde{t})-2 \eta$ and $\tilde{\xi}_{+}(\tilde{t})>\xi_{+}(\tilde{t})+2 \eta$. Now let $M_{\infty}>0$ be given. We will show that there are $R>0$, $\nu>0$ such that after a possible reduction of $V$ and $\tau>0$ holds

$$
y(t, x ; \hat{u})=Y(t, x ; \hat{u}) \quad \forall(t, x) \in S, \forall \hat{u} \in \hat{V}
$$

with $\hat{V}$ defined in ii). To this purpose we note that independent of $\nu, R$ there is $M>0$ such that $\left\|\hat{u}_{0}\right\|_{\infty}<M$ and $\left\|\hat{u}_{1}\right\|_{\infty}<M$ for all $\hat{u} \in \hat{V}$. Thus, by Theorem 3.1 i) there is $M_{y}>0$ with $\|y(. ; \hat{u})\|_{\infty} \leq M_{y}$ for all $\hat{u} \in \hat{V}$ and we can thus for convenience assume (A2') instead of (A2) ensuring with Lemma 3.3 the local Lipschitz stability of backward characteristics. Thus, if we denote by $\left(\zeta^{\tilde{t}}, v^{\tilde{t}}\right)\left(t ; x, w, u_{1}\right)$ the backward solution of (3.4) with $\left(\zeta^{\tilde{t}}, v^{\tilde{t}}\right)\left(\tilde{t} ; x, w, u_{1}\right)=(x, w)$, we can reduce $V$ and find $\varepsilon>0$ such that

$$
\zeta^{\tilde{t}}\left(0 ; x, w, \hat{u}_{1}\right) \in J, \quad \zeta^{\tilde{t}}\left(t ; x, w, \hat{u}_{1}\right)\left\{\begin{array}{l}
<\xi_{-}(t), \text { if } x \in\left[\tilde{\xi}_{-}(\tilde{t}), \xi_{-}(\tilde{t})-\eta\right] \\
>\xi_{+}(t), \text { if } x \in\left[\xi_{+}(\tilde{t})+\eta, \tilde{\xi}_{+}(\tilde{t})\right]
\end{array}, t \in[0, \tilde{t}]\right.
$$

whenever $\hat{u} \in \hat{V},|w-y(\tilde{t}, x ; u)| \leq \varepsilon$. Now we have for $I_{R}=[-R, R]$ with sufficiently large $R>0$ by Theorem 3.1 ii) the local $L^{1}$-stability estimate

$$
\|y(\tilde{t}, \cdot ; \hat{u})-y(\tilde{t}, \cdot ; u)\|_{1,\left[\tilde{\xi}_{-}(\tilde{t}), \tilde{\xi}_{+}(\tilde{t})\right]} \leq C\left(\left\|\hat{u}_{0}-u_{0}\right\|_{1, I_{R}}+\left\|\hat{u}_{1}-u_{1}\right\|_{1,[0, T] \times I_{R}}\right)
$$

for all $\hat{u} \in \hat{V}$ with $C$ only depending on $f, g, u, V, M_{\infty}$. From (4.14) and the definition of $\hat{V}$ we deduce that for $\nu>0$ small enough

$$
\underset{x \in I\left(\tilde{\xi}_{\mp}(\tilde{t}), \xi_{\mp}(\tilde{t}) \mp \eta\right)}{\operatorname{essinf}}|y(\tilde{t}, \cdot ; \hat{u})-y(\tilde{t}, \cdot ; u)|<\frac{C \nu}{\eta} \leq \varepsilon
$$

whenever $\hat{u} \in \hat{V}$. Since $y(\tilde{t}, \cdot ; \hat{u}) \in B V_{l o c}(\mathbb{R})$, we obtain by combining (4.13) and (4.15) that for any $\hat{u} \in \hat{V}$ we can find continuity points $\hat{x}_{\mp} \in I\left(\tilde{\xi}_{\mp}(\tilde{t}), \xi_{\mp}(\tilde{t}) \mp \eta\right)$ of $y(\tilde{t}, \cdot ; \hat{u})$ such that the genuine backward characteristics $\hat{\xi}_{\mp}(t)=\zeta^{\tilde{t}}\left(t ; \hat{x}_{\mp}, y\left(\tilde{t}, \hat{x}_{\mp} ; \hat{u}\right), \hat{u}_{1}\right)$ satisfy (4.13) with 
$x=\hat{x}_{\mp}, w=y\left(\tilde{t}, \hat{x}_{\mp} ; \hat{u}\right)$, respectively. Since $\hat{\xi}_{\mp}(0) \in J$, we must have $\hat{\xi}_{\mp}(0)=Z\left(\tilde{t}, \hat{x}_{\mp}, \hat{u}\right)$ and therefore $y\left(\tilde{t}, \hat{x}_{\mp} ; \hat{u}\right)=Y\left(\tilde{t}, \hat{x}_{\mp}, \hat{u}\right)$. Now any genuine backward characteristic $\xi$ of $y(\cdot ; \hat{u})$ through $(t, x) \in S$ may not leave the area confined by the genuine characteristics $\hat{\xi}_{\mp}$. Therefore, $\xi(0) \in J$ and $\xi$ must by Lemma 4.1 coincide with the unique genuine forward characteristic starting in $Z(t, x, \hat{u})$ and consequently $y(t, x ; \hat{u})=Y(t, x, \hat{u})$. Thus, (4.12) is proven.

4.2. Differentiability in the interior of rarefaction waves (case $\mathbf{R}^{c}$ ). In a second step, we look at continuity points outside the shock set that are located on a rarefaction wave. Then (3.9), (3.10) hold with $\bar{z}=x_{i}, u_{0}(\bar{z}-)<u_{0}(\bar{z}+)$, for some $i \in\{1, \ldots, N\}$, and some $\bar{w} \in] u_{0}(\bar{z}-), u_{0}(\bar{z}+)\left[\right.$. Moreover, we obtain from (3.20) by continuity $w_{-}<\bar{w}<w_{+}$and $\beta, \rho>0$ with

$$
\left.\left.\left.\frac{d}{d w} \zeta\left(t ; \bar{z}, w, u_{1}\right) \geq \beta t>0, \forall t \in\right] 0, \bar{t}\right], \forall w \in\right] w_{-}-\rho, w_{+}+\rho \stackrel{\text { def }}{=} J_{w} .
$$

Then we can locally solve (3.9) yielding with (3.10) a representation for rarefaction waves. The following Lemma is a counterpart of Lemma 4.1 for the solution of (3.9) with respect to $\bar{w}$.

LEMMA 4.5. Let $u_{0} \in P C^{1}\left(\mathbb{R} ; x_{1}, \ldots, x_{N}\right), u_{1} \in L^{\infty}\left(0, T ; C^{1}(\mathbb{R})^{m}\right)$, and let $(4.16)$ hold for some $\beta, \rho>0$. Then there is $\tau>0$ and a neighborhood $V_{1} \subset L^{\infty}\left(0, T ; C^{1}(\mathbb{R})^{m}\right)$ of $u_{1}$ such that

$$
\left.\left.\frac{d}{d w} \zeta\left(t ; \bar{z}, w, \hat{u}_{1}\right) \geq \frac{\beta}{2} t>0 \quad \forall(t, w) \in\right] 0, \bar{t}+\tau\right] \times J_{w}, \quad \forall \hat{u}_{1} \in V_{1} .
$$

Moreover, for all $\hat{u}_{1} \in V_{1}$ and all $(t, x)$ in the stripe

$$
\left.S \stackrel{\text { def }}{=}\{(t, x): t \in] 0, \bar{t}+\tau], x \in\left[\xi_{-}(t), \xi_{+}(t)\right]\right\}, \quad \xi_{\mp}(t)=\zeta\left(t ; \bar{z}, w_{\mp}, u_{1}\right)
$$

the equation

$$
x=\zeta\left(t ; \bar{z}, w, \hat{u}_{1}\right)
$$

has in $J_{w}$ a unique solution $w=W\left(t, x, \hat{u}_{1}\right)$. Set

$$
Y_{r}\left(t, x, \hat{u}_{1}\right) \stackrel{\text { def }}{=} v\left(t ; \bar{z}, W\left(t, x, \hat{u}_{1}\right), \hat{u}_{1}\right) .
$$

Then $W\left(\cdot, \hat{u}_{1}\right), Y_{r}\left(\cdot, \hat{u}_{1}\right) \in C^{0,1}(S \cap\{t \geq \tilde{t}\})$ for any $\left.\tilde{t} \in\right] 0, \bar{t}[$ and the mappings

$$
\begin{aligned}
&\left.\left(x, \hat{u}_{1}\right) \in\right] \xi_{-}(t), \xi_{+}(t)\left[\times V_{1}\right.\left.\longmapsto\left(W, Y_{r}\right)\left(t, x, \hat{u}_{1}\right), \quad t \in\right] \tilde{t}, \bar{t}+\tau[ \\
& \hat{u}_{1} \in V_{1} \longmapsto\left(W, Y_{r}\right)\left(\cdot, \hat{u}_{1}\right) \in C(S \cap\{t \geq \tilde{t}\})^{2}
\end{aligned}
$$

are continuously Fréchet differentiable. The derivatives of (4.20) are

$$
\begin{aligned}
d_{\left(x, u_{1}\right)} W\left(t, x, \hat{u}_{1}\right) \cdot\left(\delta x, \delta u_{1}\right)=\frac{\delta x-\delta \zeta\left(t ; \bar{z}, w, \hat{u}_{1} ; 0,0, \delta u_{1}\right)}{\delta \zeta\left(t ; \bar{z}, w, \hat{u}_{1} ; 0,1,0\right)} \\
\begin{aligned}
d_{\left(x, u_{1}\right)} Y_{r}\left(t, x, \hat{u}_{1}\right) \cdot\left(\delta x, \delta u_{1}\right)= & \delta v\left(t ; \bar{z}, w, \hat{u}_{1} ; 0,1,0\right) d_{\left(x, u_{1}\right)} W\left(t, x, \hat{u}_{1}\right) \cdot\left(\delta x, \delta u_{1}\right) \\
& +\delta v\left(t ; \bar{z}, w, \hat{u}_{1} ; 0,0, \delta u_{1}\right)
\end{aligned}
\end{aligned}
$$

where $w=W\left(t, x, \hat{u}_{1}\right)$ and $(\delta \zeta, \delta v)$ are given by (3.13). The derivative of (4.21) is

$$
d_{u_{1}}\left(W, Y_{r}\right)\left(\cdot, \hat{u}_{1}\right) \cdot \delta u_{1}=d_{\left(x, u_{1}\right)}\left(W, Y_{r}\right)\left(\cdot, \hat{u}_{1}\right) \cdot\left(0, \delta u_{1}\right) .
$$

Finally, there is $C>0$ such that

$$
\left|d_{u_{1}}\left(W, Y_{r}\right)\left(\cdot, \hat{u}_{1}\right) \cdot \delta u_{1}\right| \leq C t\left\|\delta u_{1}\right\|_{L^{\infty}(0, T ; C(\mathbb{R}))} \quad \text { in } S,
$$


which allows a continuous extension to $(0, \bar{z})$ by the value 0 .

Proof. Except for the last assertion the proof is very similar to the one of Lemma 4.1 and therefore omitted. For the last statement we observe that with a generic constant $C$ an application of Gronwall's Lemma to (3.13) yields $\left|\delta v\left(t ; \bar{z}, w, \hat{u}_{1} ; 0,0, \delta u_{1}\right)\right| \leq C t\left\|\delta u_{1}\right\|_{L^{\infty}(0, T ; C(\mathbb{R}))}$ and then $\left|\delta \zeta\left(t ; \bar{z}, w, \hat{u}_{1} ; 0,0, \delta u_{1}\right)\right| \leq C t^{2}\left\|\delta u_{1}\right\|_{L^{\infty}(0, T ; C(\mathbb{R}))}$ by the first equation in (3.13). Using this together with (4.17) in (4.22), (4.23) gives the asserted bound.

REMARK 4.6. By construction, $Y_{r}\left(\cdot, \hat{u}_{1}\right) \in C^{0,1}(S \cap\{t>\tilde{t}\})$ is for any $\left.\tilde{t} \in\right] 0, \bar{t}[$ on $S \cap\{t>\tilde{t}\}$ classical solution of (1.1) for the control $\hat{u}_{1} \in V_{1}$. $\square$

REMARK 4.7. It can easily be verified that $\delta Y=d_{u_{1}} Y_{r}\left(\cdot, u_{1}\right) \cdot \delta u_{1} \in C(S)$ is a broad solution of the linearized equation

$$
\begin{aligned}
& \partial_{t} \delta Y+\partial_{x}\left(f^{\prime}\left(Y_{r}\right) \delta Y\right)=g_{y}\left(t, x, Y_{r}, \hat{u}_{1}\right) \delta Y+g_{u_{1}}\left(t, x, Y_{r}, \hat{u}_{1}\right) \delta u_{1}, \quad(t, x) \in S \\
& \quad \lim _{(t, x) \in S, t \rightarrow 0} \delta Y(t, x)=0
\end{aligned}
$$

Moreover, using the same arguments as in Remark 4.3, $\delta Y$ is also a weak solution and for every domain $D \subset S$ with Lipschitz boundary and any $p \in C(D)$ with $p \in C^{0,1}(D \cap\{t \geq \tilde{t}\})$ for all $\tilde{t} \in] 0, \bar{t}$ [ the identity (4.11) holds. This follows by integrating by parts over $D \cap\{t>\tilde{t}\}$ and letting $\tilde{t} \rightarrow 0$.

Notation. Given $\bar{t}, \bar{z}, w_{-}, w_{+}$satisfying (4.16) for some $\beta, \rho>0$, we indicate by

$$
\left(Y_{r}, W, V_{1}, S(\tau), J_{w}\right)=\operatorname{YR}\left(u, \bar{t}, \bar{z},\left[w_{-}, w_{+}\right]\right)
$$

that the open interval $J_{w} \supset\left[w_{-}, w_{+}\right]$, the stripe $S=S(\tau)$, the neighborhood $V_{1}$ and the functions $Y_{r}, Z$ are obtained by applying Lemma 4.5.

Using Lemma 4.5 we obtain the following regularity result at continuity points that are located on the interior of a rarefaction wave.

LEMMA 4.8. Let $u=\left(u_{0}, u_{1}\right) \in P C^{1}\left(\mathbb{R} ; x_{1}, \ldots, x_{N}\right) \times L^{\infty}\left(0, T ; C^{1}(\mathbb{R})^{m}\right)$. Let $(\bar{t}, \bar{x}) \in \Omega_{T}$ be a point of continuity of $y=y(\cdot ; u)$ outside the shock set such that with $\bar{z}, \bar{w}$ in (3.9), (3.10) holds $u_{0}(\bar{z}-)<\bar{w}<u_{0}(\bar{z}+)$. Then the following statements are true:

i) There is a maximal nonempty open interval I such that $\{\bar{t}\} \times I$ does not contain points of the shock set and such that all backward characteristics through $(\bar{t}, x), x \in I$ meet $t=0$ in $\bar{z} . y(\bar{t}, \cdot ; u)$ is continuously differentiable on $I$.

ii) Let $J$ be an arbitrary neighborhood of $\bar{z}$. Let $\hat{I}=] x_{-}, x_{+}[$be an arbitrary interval with closure in $I$, denote by $\xi_{\mp}$ the genuine backward characteristics through $\left(\bar{t}, x_{\mp}\right)$ and by $\left.w_{\mp} \in\right] u_{0}(\bar{z}-), u_{0}(\bar{z}+)$ [ the corresponding values of $\bar{w}$ in (3.9), (3.10). Then there are $\beta>$ $0, \rho>0$ such that (4.16) is satisfied. Let $\left(Y_{r}, W, V_{1}, S(\tau), J_{w}\right)=\operatorname{YR}\left(u, \bar{t}, \bar{z},\left[w_{-}, w_{+}\right]\right)$ be obtained according to Lemma 4.5. Given $M_{\infty}>0$ and $\left.\tilde{t} \in\right] 0, \bar{t}$ there are $R>0, \nu>0$ such that after a possible reduction of $\tau$ and $V_{1}$ holds:

$$
\begin{gathered}
y(t, x ; \hat{u})=Y_{r}\left(t, x, \hat{u}_{1}\right) \quad \forall(t, x) \in S \cap\{t \geq \tilde{t}\}, \forall \hat{u} \in \hat{V}, \text { where } \\
\hat{V} \stackrel{\text { def }}{=}\left\{\left(\hat{u}_{0}, \hat{u}_{1}\right) \in L^{\infty}(\mathbb{R}) \times L^{\infty}\left(0, T ; C^{1}(\mathbb{R})^{m}\right): \hat{u}_{1} \in V_{1},\left\|\hat{u}_{0}-u_{0}\right\|_{\infty, J} \leq \nu\right. \\
\left.\left\|\hat{u}_{0}-u_{0}\right\|_{\infty, \mathbb{R} \backslash J} \leq M_{\infty},\left\|\hat{u}_{0}-u_{0}\right\|_{1,[-R, R] \backslash J} \leq \nu\right\} .
\end{gathered}
$$

Hence, the differentiability properties of $Y_{r}$ according to Lemma 4.5 hold also for $y(. ; \hat{u})$.

Proof. The proof is very similar to the proof of Lemma 4.4 and we only sketch the differences. One has to use (3.20) instead of (3.17) to obtain the applicability of Lemma 4.5. Then by backward stability characteristics $\xi$ starting in continuity points $(\bar{t}, \tilde{x})$ close to $(\bar{t}, \bar{x})$ must hit $t=0$ in $\bar{z}$, since $y(t, \xi(t))$ converges for $t \rightarrow 0, \tilde{x} \rightarrow \bar{x}$ to $\bar{w} \in] u_{0}(\bar{z}-), u_{0}(\bar{z}+)[$. Now by Lemma 4.5 the same arguments as in the proof of Lemma 4.4 can be used. Finally, one shows with the $L^{1}$-stability estimate that for sufficiently small $\nu>0, \tau>0$ and $V_{1}$ also the backward characteristics of $y(\cdot ; \hat{u}), \hat{u} \in \hat{V}$, through $(\hat{t}, \hat{x}) \in S \cap\{t \geq \tilde{t}\}$ must hit $t=0$ in $\bar{z} . \mathrm{L}$ 
5. Stability of shocks and differentiability of shock position. We turn now to the study of points $(\bar{t}, \bar{x})$ in the shock set. We will show that under the assumption

(S) Left and right states of $(\bar{t}, \bar{x})$ are of class $\mathrm{C}^{c}$ or $\mathrm{R}^{c}$ and $(\bar{t}, \bar{x})$ is no shock interaction point the following holds for quite general variations $\left(\hat{u}_{0}, \hat{u}_{1}\right)$ of $\left(u_{0}, u_{1}\right)$ :

- The shock is stable and separates states that coincide locally with representations $Y$ or $Y_{r}$ obtained by Lemma 4.1 or 4.5 , respectively.

Moreover, if $g(t, x, y, u)$ is affine linear with respect to $y$ then

- The shock position depends Fréchet differentiable on $\left(\hat{u}_{0}, \hat{u}_{1}\right)$.

- The sensitivity of the shock position w.r.t. $\left(\hat{u}_{0}, \hat{u}_{1}\right)$ can be explicitly computed by means of an adjoint state.

We will show in Theorem 11.2 that assumption (S) holds for almost all $\bar{t}$ under slightly stronger regularity assumptions on $\left(u_{0}, u_{1}\right)$. The restriction to source terms $g$ that are affine linear with respect to $y$ could be dropped without major changes in our analysis if the stability results for the adjoint equation (1.6) can be extended to a discontinuous zeroth order coefficient $g_{y}\left(t, x, y, u_{1}\right)$.

5.1. Stability and structure of shocks. The nonlinear stability of shock waves for a single convex conservation law (i.e. $f^{\prime \prime}>0, g \equiv 0$ ) under variations of the initial data in the Schwartz space $\mathcal{S}$ was shown by Golubitsky and Schaeffer [13]. However, there seem to be no results in the literature on the nonlinear stability of shocks under perturbations of initial data and source term that are directly applicable in our framework. In the following Lemma we give a stability result of shock waves based on generalized characteristics that fits our purposes.

For concreteness we consider a shock point $(\bar{t}, \bar{x})$ of class $\mathrm{C}^{c} \mathrm{C}^{c}$.

LEMMA 5.1. Let $u=\left(u_{0}, u_{1}\right) \in P C^{1}\left(\mathbb{R} ; x_{1}, \ldots, x_{N}\right) \times L^{\infty}\left(0, T ; C^{1}(\mathbb{R})^{m}\right)$ and let $(\bar{t}, \bar{x}) \in \Omega_{T}$ be a point of discontinuity of $y(. ; u)$ on a shock curve $\eta(t)$ that is no shock interaction point. Denote by $\xi_{\mp}(t)$ the minimal and maximal backward characteristic through $(\bar{t}, \bar{x})$ and set $\bar{z}^{\mp}=\xi_{\mp}(0)$. We assume that $(\bar{t}, \bar{x})$ is of class $C^{c} C^{c}$, i.e. $\bar{z}^{\mp} \neq x_{i}, 1 \leq i \leq N$, and (3.17) holds with $\bar{z}=\bar{z}^{\mp}$ for some $\beta>0$. Then there are $z_{-}^{\mp}<\bar{z}^{\mp}<z_{+}^{\mp}, \rho>0$ and $\beta>0$ such that (4.1) holds.

Let $\left(Y_{\mp}, Z_{\mp}, V_{\mp}, S_{\mp}(\tau), J_{\mp}\right)=\mathrm{YC}\left(u, \bar{t},\left[z_{-}^{\mp}, z_{+}^{\mp}\right]\right)$ be obtained by Lemma 4.1 with $z_{\mp}^{-}, z_{\mp}^{+}$ close enough to $\bar{z}^{\mp}$, respectively. After a possible reduction of $V_{\mp}, \tau>0$ there is a neighborhood $I=] x_{-}, x_{+}[$of $\bar{x}$ such that the following holds:

i) $y(\cdot ; u)$ is locally given by

$$
y(t, x ; u)= \begin{cases}Y_{-}(t, x, u), & (t, x) \in S_{-} \cap\{x<\eta(t)\} \\ Y_{+}(t, x, u), & (t, x) \in S_{+} \cap\{x>\eta(t)\}\end{cases}
$$

ii) Let $M_{\infty}>0$ be given. Then there are $R>0, \nu>0$ such that for

$$
\begin{array}{r}
\hat{V}=\left\{\left(\hat{u}_{0}, \hat{u}_{1}\right) \in L^{\infty}(\mathbb{R}) \times L^{\infty}\left(0, T ; C^{1}(\mathbb{R})^{m}\right):\left(\left.\hat{u}_{0}\right|_{J_{\mp}}, \hat{u}_{1}\right) \in V_{\mp},\right. \\
\left.\left\|\hat{u}_{0}\right\|_{\infty} \leq M_{\infty},\left\|\hat{u}_{0}-u_{0}\right\|_{1,[-R, R]} \leq \nu\right\}
\end{array}
$$

equipped with the norm

$$
\left\|\left(\hat{u}_{0}, \hat{u}_{1}\right)\right\|_{\mathcal{V}} \stackrel{\text { def }}{=}\left\|\hat{u}_{0}\right\|_{C^{1}\left(J_{-} \cup J_{+}\right)}+\left\|\hat{u}_{0}\right\|_{1,\left[\bar{z}^{-}, \bar{z}^{+}\right]}+\left\|\hat{u}_{1}\right\|_{L^{\infty}\left(0, T ; C^{1}(\mathbb{R})\right)}
$$

there is a Lipschitz continuous function

$$
x_{s}: \hat{u} \in\left(\hat{V},\|\cdot\|_{\mathcal{V}}\right) \longmapsto x_{s}(\hat{u})
$$

with $x_{s}(u)=\bar{x}$ such that for all $\hat{u}=\left(\hat{u}_{0}, \hat{u}_{1}\right) \in \hat{V}$ holds

$$
y(\bar{t}, x ; \hat{u})= \begin{cases}Y_{-}\left(\bar{t}, x,\left.\hat{u}_{0}\right|_{J_{-}}, \hat{u}_{1}\right), & x \in] x_{-}, x_{s}(\hat{u})[ \\ Y_{+}\left(\bar{t}, x,\left.\hat{u}_{0}\right|_{J_{+}}, \hat{u}_{1}\right), & x \in] x_{s}(\hat{u}), x_{+}[\end{cases}
$$

Hereby, $\hat{u} \in\left(\hat{V},\|\cdot\|_{\mathcal{V}}\right) \longmapsto Y_{\mp}\left(\bar{t}, \cdot,\left.\hat{u}_{0}\right|_{J_{\mp}}, \hat{u}_{1}\right) \in C(I)$ are continuously Fréchet differentiable. 
Proof. $(\bar{t}, \bar{x})$ is a point of discontinuity on a shock curve $\eta$. Thus, we have by the entropy condition $y(\bar{t}, \eta(\bar{t})-; u)>y(\bar{t}, \eta(\bar{t})+; u)$. Since (3.17) holds for $\bar{z}=\bar{z}^{\mp}$ we obtain by continuity $\beta>0, J_{\mp}$ such that (4.1) holds. Now Lemma 4.1 yields as asserted $\left(Y_{\mp}, Z_{\mp}, V_{\mp}, S_{\mp}(\tau), J_{\mp}\right)=\mathrm{YC}\left(u, \bar{t},\left[z_{-}^{\mp}, z_{+}^{\mp}\right]\right)$. By Lemma 4.1 and the definition of $\hat{V}$ in ii)

$$
\left(\hat{u}_{0}, \hat{u}_{1}\right) \in\left(\hat{V},\|\cdot\|_{\mathcal{V}}\right) \longmapsto Y_{\mp}\left(\bar{t}, \cdot,\left.\hat{u}_{0}\right|_{J_{\mp}}, \hat{u}_{1}\right) \in C(I)
$$

are obviously continuously Fréchet differentiable.

i): Since $(\bar{t}, \bar{x})$ is no shock interaction point, we know from [8] that $\bar{t}$ is a continuity point of $t \longmapsto y(t, \eta(t) \pm ; u)$. Then by [8, Thm. 4.5] $(\bar{t}, \bar{x})$ is a continuity point of $y(\cdot ; u)$ relative to the sets $\mathcal{L}_{-}=\{(t, x): x<\eta(t)\}, \mathcal{L}_{+}=\{(t, x): x>\eta(t)\}$, the limit being $y(\bar{t}, \eta(\bar{t}) \mp ; u)$. The extreme backward characteristics $\xi_{\mp}(t)$ through $(\bar{t}, \bar{x})$ satisfy (3.4) and $\xi_{\mp}(0)=\bar{z}_{\mp} \in J_{\mp}$. Using the backward stability of solutions of (3.4) we find $\delta>0$ such that the genuine backward characteristic through any continuity point in $Q_{\mp} \stackrel{\text { def }}{=} \mathcal{L}_{\mp} \cap\left\{\|(t, x)-(\bar{t}, \bar{x})\|_{\infty} \leq \delta\right\}$ meets $t=0$ in $J_{\mp}$, respectively. Hence, we must have $y(t, x ; u)=Y_{\mp}(t, x, u)$ for any continuity point in $Q_{\mp}$ and since the continuity points are dense, for all points in $Q_{\mp}$ by our choice of $y$. The same is clearly true on the domain covered by these backward characteristics. Possibly after reducing $\tau>0$ and choosing $z_{\mp}^{-}, z_{\mp}^{+}$closer to $\bar{z}_{\mp}$, respectively, we achieve that $S_{\mp} \cap \mathcal{L}_{\mp}$ is covered by the backward characteristics through $Q_{\mp}$. Hence, i) is proven.

ii): For notational convenience we write $y$ instead of $y(\cdot ; u)$. By the definition of $S_{\mp}$ it is clear that for sufficiently small $\tau>0, \sigma>0$ and $x_{\mp}=\bar{x} \mp \sigma$ holds $\left.Q \stackrel{\text { def }}{=}\right] \bar{t}-\tau, \bar{t}+$ $\tau[\times] x_{-}, x_{+}\left[\subset S_{-} \cap S_{+}\right.$. Set $\left.I \stackrel{\text { def }}{=}\right] x_{-}, x_{+}\left[\right.$. As in the proof of Lemma 4.4 there is $M_{y}>0$ with $\|y(\cdot ; \hat{u})\|_{\infty} \leq M_{y}$ for all $\hat{u} \in \hat{V}$ and we may choose $M_{y}$ such that in addition $\left|Y_{\mp}(\cdot, \hat{u})\right| \leq M_{y}$ on $S_{\mp}$.

By continuity, we may reduce $\tau$ such that for some $\Delta>0$

$$
y(t, \eta(t)-)-y(t, \eta(t)+) \geq 2 \Delta>0 \quad \forall t \in] \bar{t}-\tau, \bar{t}+\tau[.
$$

Since $f^{\prime \prime} \geq m_{f^{\prime \prime}}>0$, we have for $|t-\bar{t}|<\tau$

$$
\dot{\eta}(t)=\frac{f(y(t, \eta(t)-))-f(y(t, \eta(t)+))}{y(t, \eta(t)-)-y(t, \eta(t)+)}\left\{\begin{array}{l}
\leq f^{\prime}(y(t, \eta(t)-))-m_{f^{\prime \prime}} \Delta \\
\geq f^{\prime}(y(t, \eta(t)+))+m_{f^{\prime \prime}} \Delta
\end{array}\right.
$$

In particular, $\dot{\eta}(t)$ is continuous in $\bar{t}$ and we may reduce $V_{\mp}, \nu, \tau, \sigma$ such that with $\varepsilon>0$ holds

$$
\begin{array}{r}
f^{\prime}\left(Y_{-}(t, x, \hat{u})\right) \geq \max _{|t-\bar{t}|<\tau} \dot{\eta}(t)+\varepsilon \\
Y_{-}(t, x, \hat{u})-Y_{+}(t, x, \hat{u}) \geq \Delta
\end{array}
$$

for all $(t, x, \hat{u}) \in Q \times \hat{V}$. Finally, reduce $\tau>0$ such that $\eta(t) \in I$ and

$$
\left|\dot{\eta}(t)-\max _{|t-\bar{t}|<\tau} \dot{\eta}(t)\right|<\frac{m_{f^{\prime \prime} \varepsilon}}{8 M_{f^{\prime \prime}}}=: \hat{\varepsilon}
$$

for all $t,|t-\bar{t}|<\tau$, where $M_{f^{\prime \prime}} \stackrel{\text { def }}{=} \sup _{|y| \leq M_{y}} f^{\prime \prime}(y)$.

The following property of genuine backward characteristics will be crucial: Let $\zeta(t)$ be a backward characteristic such that

$$
|\zeta(\hat{t})-\eta(\hat{t})|<\delta, \quad \dot{\zeta}(\hat{t})<\dot{\eta}(\hat{t})-2 \hat{\varepsilon} \quad \text { for some } \hat{t} \in[\bar{t}-\tau / 2, \bar{t}] .
$$

From (3.4) we get a constant $M>0$ with

$$
|\ddot{\zeta}(t)| \leq M
$$


and we can reduce $\tau>0$ such that $M \tau<\hat{\varepsilon} / 2$. Then $\zeta(t)-\eta(t)>\delta$ for $t=\bar{t}-\tau$ if $\delta<\hat{\varepsilon} \tau / 8$. In fact, we get with $s=\min _{|t-\bar{t}|<\tau} \dot{\eta}(t)$ and $t=\bar{t}-\tau$ by using (5.5)

$$
\begin{aligned}
\zeta(t)-\eta(t) & \geq \zeta(\hat{t})-\eta(\hat{t})+(s-\dot{\zeta}(\hat{t}))(\hat{t}-t)-\frac{M}{2}(\hat{t}-t)^{2} \\
& \geq-\delta+(s-\dot{\eta}(\hat{t})+2 \hat{\varepsilon})(\hat{t}-t)-\frac{M}{2}(\hat{t}-t)^{2} \\
& \geq-\delta+(-\hat{\varepsilon}+2 \hat{\varepsilon}-M \tau) \frac{\tau}{2} \geq \frac{\hat{\varepsilon} \tau}{4}-\delta>\delta .
\end{aligned}
$$

Fix these $\tau$ and $\delta$ and set $Q_{\delta} \stackrel{\text { def }}{=} Q \cap\{(t, x):|x-\eta(t)|<\delta\}$. Using a finite covering of $\left(Q^{c l} \cap \mathcal{L}_{\mp}\right) \backslash Q_{\delta}$ with the stripes $S$ obtained by Lemma 4.4 for varying $t$ we may reduce $V_{\mp}$, $\nu$ such that for all $\hat{u} \in \hat{V}$

$$
y(t, x ; \hat{u})=Y_{\mp}\left(t, x,\left.\hat{u}_{0}\right|_{J_{\mp}}, \hat{u}_{1}\right) \quad \forall(t, x) \in\left(Q \cap \mathcal{L}_{\mp}\right) \backslash Q_{\delta},
$$

respectively. For the rest of the proof it is convenient to set $Y_{\mp}(\cdot, \hat{u})=Y_{\mp}\left(\cdot,\left.\hat{u}_{0}\right|_{J_{\mp}}, \hat{u}_{1}\right)$ for $\hat{u} \in \hat{V}$ on $S_{\mp}$.

Assume, that (5.2) does not hold for all $\hat{u} \in \hat{V}$. Then there is $\hat{u} \in \hat{V}$ and without restriction a continuity point $\hat{x},|\hat{x}-\bar{x}|<\delta$ with $y(\bar{t}, \hat{x} ; \hat{u}) \neq Y_{\mp}(\bar{t}, \hat{x}, \hat{u})$ and thus $\hat{\xi}(0)<\inf J_{+}$for the genuine backward characteristic $\hat{\xi}$ through $(\bar{t}, \hat{x})$. For convenience we set $\hat{y} \stackrel{\text { def }}{=} y(\cdot ; \hat{u})_{\dot{\tilde{y}}}$ Let $\tilde{x}$ be the infimum of these $\hat{x}$. $(\bar{t}, \tilde{x})$ can not be a continuity point, since else by continuity $\tilde{\xi}(0) \in J_{-}$ for the backward characteristic $\tilde{\xi}$ through $(\bar{t}, \tilde{x})$ and by Lemma 4.4 thus $\hat{y}(\bar{t}, x)=Y_{-}(\bar{t}, x, \hat{u})$ for $x$ close enough to $\tilde{x}$ which is a contradiction. Thus, $(\bar{t}, \tilde{x})$ lies on a shock. We set $\tilde{\eta}(\bar{t})=\tilde{x}$. By construction the maximal backward characteristic $\tilde{\xi}_{+}$through $(\bar{t}, \tilde{x})$ satisfies $\sup J_{-}<\tilde{\xi}_{+}(0)<\inf J_{+}$. Since genuine characteristics may intersect only at their endpoints, we have by (5.7) necessarily $\left(t, \tilde{\xi}_{+}(t)\right) \in Q_{\delta}$ for $\bar{t}-\tau<t \leq \bar{t}$. Moreover, it is obvious from $\sup J_{-}<\tilde{\xi}_{+}(0)<\inf J_{+}$that $\hat{y}\left(t, \tilde{\xi}_{+}(t)\right) \neq Y_{\mp}\left(t, \tilde{\xi}_{+}(t), \hat{u}\right)$ for $\bar{t}-\tau<t \leq \bar{t}$. Thus, we find as above for all these $t$ a discontinuity point $\tilde{\eta}(t)$ with left state $Y_{-}(t, \tilde{\eta}(t), \hat{u})$. Now the unique forward characteristic through $(t-\tau, \tilde{\eta}(t-\tau))$ must be a shock and it must coincide with $\tilde{\eta}$, since every $(t, \tilde{\eta}(t))$ lies on a shock with left state $Y_{-}(t, \tilde{\eta}(t), \hat{u})$ and can therefore not be shock generation points. Hence, $\tilde{\eta}(t)$ is a shock with left state $Y_{-}(t, \tilde{\eta}(t), \hat{u})$ and we must have $|\tilde{\eta}(t)-\eta(t)|<\delta$ for $\bar{t}-\tau<t \leq \bar{t}$, because $\tilde{\eta}$ may not enter the domain of continuity. Hence, there must exist $\hat{t} \in[\bar{t}-\tau / 2, \bar{t}]$ with

$$
\dot{\tilde{\eta}}(\hat{t}) \leq \max _{|t-\bar{t}|<\tau} \dot{\eta}(t)+\hat{\varepsilon}
$$

because $\hat{\varepsilon} \tau / 2>2 \delta$ by the choice of $\delta$. Set $y_{\mp}=\hat{y}(\hat{t}, \tilde{\eta}(t) \mp)$. We show that necessarily $f^{\prime}\left(y_{+}\right) \leq s-2 \hat{\varepsilon}$ with $s=\min _{|t-\bar{t}|<\tau} \dot{\eta}(t)$ as defined above. If not, then we have by (5.3)

$$
h \stackrel{\text { def }}{=} f^{\prime}\left(y_{-}\right)-f^{\prime}\left(y_{+}\right) \geq s+\varepsilon-(s-2 \hat{\varepsilon}) \geq \varepsilon+2 \hat{\varepsilon}>\varepsilon .
$$

Moreover, $y_{-}-y_{+} \geq \frac{h}{M_{f^{\prime \prime}}}$ and thus with $f^{\prime \prime} \geq m_{f^{\prime \prime}}$, and (5.5)

$$
\dot{\tilde{\eta}}(\hat{t})=\frac{f\left(y_{-}\right)-f\left(y_{+}\right)}{y_{-}-y_{+}} \geq f^{\prime}\left(y_{+}\right)+\frac{m_{f^{\prime \prime}} h}{2 M_{f^{\prime \prime}}}>s-2 \hat{\varepsilon}+\frac{m_{f^{\prime \prime} h}}{2 M_{f^{\prime \prime}}}>\max _{|t-\bar{t}|<\tau} \dot{\eta}(t)-3 \hat{\varepsilon}+4 \hat{\varepsilon} .
$$

This contradicts (5.8). Thus, the maximal backward characteristic $\zeta$ through $(\hat{t}, \tilde{\eta}(\hat{t}))$ satisfies the scenario (5.6), since $\dot{\zeta}(\hat{t})=f^{\prime}\left(y_{+}\right) \leq s-2 \hat{\varepsilon}$. Therefore, it would hold $\zeta(\hat{t}-\tau)-\eta(\hat{t}-\tau)>$ $\delta$. This is a contradiction, since then $\zeta$ would intersect $\tilde{\xi}_{+}$. Hence, the assumption was wrong and (5.2) is shown. It remains to show that $\left.\hat{u} \in\left(\hat{V},\|\cdot\|_{\mathcal{V}}\right)\right) \mapsto x_{s}(\hat{u})$ is Lipschitz. But this follows directly from (5.2), (5.4) and the $L^{1}$-stability according to Theorem $3.1 \mathrm{ii}$ ). The continuous differentiability of $\hat{u} \longmapsto Y_{\mp}(\bar{t}, \cdot, \hat{u}) \in C(I)$ was already shown at the beginning of the proof.

Very similar results can be obtained if left and/or right states are rarefaction waves, i.e. in the cases $\mathrm{C}^{c} \mathrm{R}^{c}, \mathrm{R}^{c} \mathrm{C}^{c}, \mathrm{R}^{c} \mathrm{R}^{c}$. See Corollary 5.3 below. 
5.2. Differentiability of the shock position and an adjoint formula. In this section we state a differentiability result for the shock position $x_{s}$ of Lemma 5.1 and derive an explicit formula for the derivative by using an appropriate adjoint state. The advantage of the adjoint approach lies in the fact that we do not have to impose regularity assumptions on $y$ for $t<\bar{t}$.

LEMMA 5.2. With the assumptions and notations of Lemma 5.1 holds:

i) Assume that $g$ is affine linear w.r.t. $y$. Then $x_{s}: \hat{u} \in\left(\tilde{V},\|\cdot\|_{\tilde{\mathcal{V}}}\right) \longmapsto x_{s}(\hat{u})$ is continuously Fréchet differentiable and denoting by $\hat{\xi}_{\mp}$ the minimal/maximal backward characteristic of $\hat{y}=y(\cdot ; \hat{u})$ through $\left(\bar{t}, x_{s}(\hat{u})\right)$ and by $D$ the domain confined by them, one has

$$
d_{u} x_{s}(\hat{u}) \cdot\left(\delta u_{0}, \delta u_{1}\right)=\left(p(0, \cdot), \delta u_{0}\right)_{2,\left[\hat{\xi}_{-}(0), \hat{\xi}_{+}(0)\right]}+\left(p g_{u_{1}}\left(t, x, \hat{y}, \hat{u}_{1}\right), \delta u_{1}\right)_{2, D}
$$

where $p \in C_{\text {loc }}^{0,1}(] 0, \bar{t}[\times \mathbb{R})$ is the reversible solution of the adjoint equation

$$
\partial_{t} p+f^{\prime}(\hat{y}) \partial_{x} p=-g_{y}\left(t, x, \hat{y}, \hat{u}_{1}\right) p, \quad p(\bar{t}, \cdot)=1 /\left[y\left(\bar{t}, x_{s}(\hat{u}) ; \hat{u}\right)\right] .
$$

Hereby, $\left(\tilde{V},\|\cdot\|_{\tilde{\mathcal{V}}}\right)$ is defined as follows: Set $J_{0}=\left[\bar{z}_{-}, \bar{z}_{+}\right] \backslash\left(J_{-} \cup J_{+}\right)$and let $J_{a} \subset J_{0}$ be an open set that contains only entropy-admissible discontinuities of $u_{0}$. Finally, let $J \subset J_{a}$ be an open set with $J^{c l} \subset J_{a}$ and set $J_{r}=J_{0} \backslash J$. Define with $\hat{V}$ from Lemma 5.1

$$
\tilde{V} \stackrel{\text { def }}{=}\left\{\left(\hat{u}_{0}, \hat{u}_{1}\right) \in \hat{V}:\left.\partial_{x}\left(\hat{u}_{0}-u_{0}\right)\right|_{J_{a}} \leq M_{L}\right\}
$$

for some fixed $M_{L}>0$ equipped with the norm

$$
\left\|\left(\hat{u}_{0}, \hat{u}_{1}\right)\right\|_{\tilde{\mathcal{V}}} \stackrel{\text { def }}{=}\left\|\hat{u}_{0}\right\|_{C^{1}\left(J_{-} \cup J_{+}\right)}+\left\|\hat{u}_{0}\right\|_{\infty, J_{r}}+\left\|\hat{u}_{0}\right\|_{1, J}+\left\|\hat{u}_{1}\right\|_{L^{\infty}\left(0, T ; C^{1}(\mathbb{R})\right)} .
$$

ii) If $g$ does not depend on $y$ then the same holds for $x_{s}: \hat{u} \in\left(\hat{V},\|\cdot\|_{\mathcal{V}}\right) \longmapsto x_{s}(\hat{u})$ in (5.1) and the reversible solution of $(5.9)$ is simply $p \equiv 1 /\left[y\left(\bar{t}, x_{s}(\hat{u}) ; \hat{u}\right)\right]$.

The proof of ii) requires an existence and stability result for backward transport equations of the form (5.9) that will be provided in the following section. The essential feature of these equations is that the coefficient $f^{\prime}(\hat{y})$ satisfies by $f^{\prime \prime}>0$ and Oleinik's entropy condition (3.2) a one-sided Lipschitz condition of the form

$$
\left.\partial_{x} f^{\prime}(\hat{y}(t, \cdot)) \leq \frac{C}{t+1 / M}, \quad t \in\right] 0, T[
$$

where $M \in\left[M_{c r}, \infty\right]$ is such that $\partial_{x} \hat{u}_{0} \leq M$.

If the left and/or right state of the shock is a rarefaction wave then an analog of Lemmas 5.1, 5.2 holds:

COROLLARY 5.3. If with the notations of Lemmas 5.1, 5.2 holds $\bar{z}^{-}=x_{i}$ and $u_{0}\left(x_{i}-\right)<$ $\bar{w}<u_{0}\left(x_{i}+\right)$ with $\bar{w}$ according to (3.9), and if (3.20) holds for the left-sided derivative with $\bar{z}=\bar{z}^{-}$then the results of Lemmas 5.1, 5.2 remain true with the following modifications: Let $\left(Y_{-}, W, V_{1}, S_{-}(\tau), J_{w}\right)=\operatorname{YR}\left(u, \bar{t}, \bar{z}^{-},\left[w_{-}, w_{+}\right]\right)$with appropriate $w_{-}<\bar{w}<w_{+}$ according to Lemma 4.5. Set $J_{-}=\emptyset$, and define for an arbitrary open interval $J_{1}$ containing $\bar{z}^{-}$and $\nu>0$ sufficiently small $V_{-}$as

$$
V_{-} \stackrel{\text { def }}{=}\left\{\hat{u}_{0} \in L^{\infty}\left(J_{1}\right):\left\|\hat{u}_{0}-u_{0}\right\|_{\infty}<\nu\right\} \times V_{1} .
$$

Finally, choose for $J_{0}$ in Lemma 5.2 i) an arbitrary open interval containing $\bar{z}^{-}$.

An analog result holds if the right state is a rarefaction wave, i.e. $\bar{z}^{+}=x_{i}, u_{0}\left(x_{i}-\right)<\bar{w}<$ $u_{0}\left(x_{i}+\right)$. 
6. The adjoint equation. We consider the backward problem for a transport equation

$$
\partial_{t} p+a \partial_{x} p=-b p, \quad p(T, \cdot)=p^{T}
$$

with $a \in L^{\infty}\left(\Omega_{T}\right), \partial_{x} a(t, \cdot) \leq \alpha(t), \alpha \in L^{1}(] 0, T[), b \in L^{\infty}\left(0, T ; C^{0,1}(\mathbb{R})\right)$. It is well known [7] that under this one-sided Lipschitz condition on $a$ for any $p^{T} \in \operatorname{Lip}(\mathbb{R})$ there exists a Lipschitz continuous solution to (6.1) which is not necessarily unique. For the case $b \equiv 0$ the stability of $p$ w.r.t. $a$ for the special class of reversible solutions was extensively studied by Bouchut and James in the recent paper [1]. We will use the results of [1] to study the stability w.r.t. $a$ and $b$. Due to space limitations we will restrict ourselves to summarize the definition of reversible solutions for (6.1) and the necessary existence, stability and regularity properties ${ }^{1}$. A detailed analysis will be given in a forthcoming paper. Our results extend the stability results in [1] to the case $b \neq 0$ which is not straightforward, since the definition of reversible solutions in [1] can not be directly used.

Denote by $\mathcal{L}_{h}$ the space of Lipschitz continuous solutions to

$$
\partial_{t} p+a \partial_{x} p=0,(t, x) \in \Omega_{T}
$$

We recall the following definition of [1]:

Definition 6.1. $p \in \mathcal{L}_{h}$ is called reversible solution of (6.2) if there exist $p_{1}, p_{2} \in \mathcal{L}_{h}$ such that $\partial_{x} p_{1} \geq 0, \partial_{x} p_{2} \geq 0$ and $p=p_{1}-p_{2}$.

Then the following holds [1]:

THEOREM 6.2. Let $a \in L^{\infty}\left(\Omega_{T}\right), \partial_{x} a(t, \cdot) \leq \alpha(t), \alpha \in L^{1}(] 0, T[)$. Then for any $p^{T} \in \operatorname{Lip}(\mathbb{R})$ there exists a unique reversible solution $p$ of $(6.2)$ with $p(T, \cdot)=p^{T}$. Moreover,

$$
\|p(t, \cdot)\|_{\infty, I} \leq\left\|p^{T}\right\|_{\infty, J}, \quad\left\|\partial_{x} p(t, \cdot)\right\|_{\infty, I} \leq e^{\int_{t}^{T} \alpha}\left\|\partial_{x} p^{T}\right\|_{\infty, J}
$$

with $I=] x_{1}, x_{2}[, J=] x_{1}-\|a\|_{\infty}(T-t), x_{2}+\|a\|_{\infty}(T-t)[$.

This concept of reversible solution is not directly extendible to the case $b \neq 0$. However, a natural extension can be obtained by using the generalized backward flow associated with $a$ :

Definition 6.3. Let $D_{b}=\left\{(s, t) \in \mathbb{R}^{2}: 0 \leq t \leq s \leq T\right\}$. Then the generalized backward flow $X: D_{b} \times \mathbb{R} \longrightarrow \mathbb{R}$ is defined by the requirement that for any $\left.\left.s \in\right] 0, T\right]$ $X(s ; \cdot, \cdot)$ is the unique reversible solution to

$$
\left.\partial_{t} X+a \partial_{x} X=0, \quad(t, x) \in\right] 0, s[\times \mathbb{R}, \quad X(s ; s, x)=x, \quad x \in \mathbb{R} .
$$

Moreover, we set $X(0 ; 0, x)=x$.

One can show [1] that $\partial_{x} X \geq 0$ and $x \longmapsto X(s ; t, x)$ is surjective for all $(s, t) \in D_{b}$ and that for all $0 \leq t \leq \sigma \leq s \leq T$ and $x \in \mathbb{R}$ the composition formula

$$
X(s ; \sigma, X(\sigma ; t, x))=X(s ; t, x)
$$

is satisfied. Moreover, for all $(t, x) \in \Omega_{T}$ holds [1]

$$
\left.\partial_{s} X(s ; t, x) \in[a(s, X(s ; t, x)+), a(s, X(s ; t, x)-)] \quad \text { for a.a. } s \in\right] t, T[.
$$

Note that by the one-sided Lipschitz condition $\partial_{x} a(t, \cdot) \leq \alpha(t), \alpha \in L^{1}(] 0, T[)$, holds $a(t, \cdot) \in$ $B V_{l o c}(\mathbb{R})$ for a.a. $t$ and thus the left- and right-sided limits in (6.4) exist.

REMARK 6.4. Since $X(t ; t, x)=x, X(\cdot ; t, x)$ is by (6.4) the unique forward characteristic through $(t, x)$ in the sense of Filippov. Thus, if $y$ is the entropy solution of (1.1) and $a=f^{\prime}(y)$ then $X(\cdot ; t, x)$ is the generalized forward characteristic through $(t, x)$.

\footnotetext{
${ }^{1}$ For convenience the proofs are added in the Appendix
} 
Finally, it is shown in [1] that for $b \equiv 0$ the reversible solution of (6.1) is given by $p(t, x)=$ $p^{T}(X(T ; t, x))$ and is thus the broad solution along the generalized characteristics. This leads us to the following definition of reversible solutions for (6.1).

Definition 6.5. Let $a \in L^{\infty}\left(\Omega_{T}\right), \partial_{x} a(t, \cdot) \leq \alpha(t), \alpha \in L^{1}(] 0, T[)$, and $b \in$ $L^{\infty}\left(0, T ; C^{0,1}(\mathbb{R})\right)$. Then a reversible solution of $(6.1)$ is defined as follows. For any $z \in \mathbb{R}$ define $p(t, X(t ; 0, z))$ as solution of

$$
\begin{aligned}
p(T, X(T ; 0, z)) & =p^{T}(X(T ; 0, z)) \\
\frac{d}{d t} p(t, X(t ; 0, z)) & =-b(t, X(t ; 0, z)) p(t, X(t ; 0, z)), t \in[0, T] .
\end{aligned}
$$

REMARK 6.6. Since by (6.3) with $x=X(s ; 0, z)$ holds $X(t ; s, x)=X(t ; 0, z)$ for $s \leq t \leq T$, (6.5) implies that for all $0 \leq s<T$ and $x \in \mathbb{R}$

$$
\begin{aligned}
p(T, X(T ; s, x)) & =p^{T}(X(T ; s, x)) \\
\frac{d}{d t} p(t, X(t ; s, x)) & =-b(t, X(t ; s, x)) p(t, X(t ; s, x)), t \in[s, T] .
\end{aligned}
$$

This shows with (6.4) that the value of $p(s, x)$ depends only on the values of $a$ and $b$ in the triangle $\left\{(t, z) \in \Omega_{T}: t \in[s, T], z \in\left[x-\|a\|_{\infty}(t-s), x+\|a\|_{\infty}(t-s)\right]\right\}$.

With this definition of reversible solutions we have the following existence and uniqueness result.

THEOREM 6.7. Let $a \in L^{\infty}\left(\Omega_{T}\right), \partial_{x} a(t, \cdot) \leq \alpha(t)$ with $\alpha \in L^{1}(] 0, T[)$ and $b \in$ $L^{\infty}\left(0, T ; C^{0,1}(\mathbb{R})\right)$. Then for all $p^{T} \in C^{0,1}(\mathbb{R})$ there exists a unique reversible solution $p$ of (6.1). Moreover, $p \in C^{0,1}\left(\Omega_{T}\right)$ and $p$ solves (6.1) a.e. on $\Omega_{T}$.

Furthermore, for all $t \in[0, T], z_{1}<z_{2}$ and $0 \leq t_{1}<t_{2} \leq T$ holds with $I=\left[z_{1}, z_{2}\right]$, $J=\left[z_{1}-\|a\|_{\infty}(T-t), z_{2}+\|a\|_{\infty}(T-t)\right]$

$$
\begin{aligned}
\|p(t, \cdot)\|_{\infty, I} & \leq\left\|p^{T}\right\|_{\infty, J} e^{\|b\|_{\infty}(T-t)}, \\
\left\|p\left(t_{2}, \cdot\right)-p\left(t_{1}, \cdot\right)\right\|_{1, I} & \leq\left(t_{2}-t_{1}\right) C
\end{aligned}
$$

where $C$ depends only on $I,\left\|p^{T}\right\|_{C^{0,1}(J)},\|a\|_{\infty,[0, T] \times J},\|b\|_{L^{\infty}\left(0, T ; C^{0,1}(J)\right)}$. Finally, one has $p \in H^{1,1}(] 0, T[\times I)$ where $\|p\|_{\left.H^{1,1}(] 0, T \times X I\right)}$ does not depend on $\alpha$.

The proof can be obtained by using the properties of the backward flow $X$. We leave the details to a forthcoming paper. $^{2}$

A key point for our analysis is the following stability result.

THEOREM 6.8. Let $\left(a_{n}\right)$ be a bounded sequence in $L^{\infty}\left(\Omega_{T}\right)$ with $a_{n} \rightarrow$ a in $L^{\infty}\left(\Omega_{T}\right)$ weak $^{*}, \partial_{x} a(t, \cdot) \leq \alpha(t), \alpha \in L^{1}(] 0, T\left[\right.$ and $\partial_{x} a_{n}(t, \cdot) \leq \alpha_{n}(t),\left(\alpha_{n}\right)$ bounded in $L^{1}(] 0, T[)$. Let $\left(b_{n}\right)$ be a bounded sequence in $L^{\infty}\left(0, T ; C^{0,1}(\mathbb{R})\right)$ with $b_{n} \rightarrow b$ in $L^{\infty}(0, T ; C(\mathbb{R}))$, $b \in L^{\infty}\left(0, T ; C^{0,1}(\mathbb{R})\right)$. Finally, let $p_{n}^{T}$ be bounded in $C^{0,1}(\mathbb{R})$ with $p_{n}^{T} \rightarrow p^{T}$ in $C(\mathbb{R})$. Then the reversible solutions $p_{n}$ of

$$
\partial_{t} p_{n}+a_{n} \partial_{x} p_{n}=-b_{n} p_{n}, \quad p_{n}(T, \cdot)=p_{n}^{T}
$$

converge for any $R>0$ in $C([0, T] \times[-R, R])$ to the reversible solution $p$ of $(6.1)$.

The proof can be obtained by using the following stability result of [1]: if $X$ and $X_{n}$ denote the backward flows according to Definition 6.3 for $a$ and $a_{n}$, respectively. Then by [1] $X_{n} \rightarrow X$ in $C\left(D_{b} \times[-R, R]\right)$ for any $R>0$. Again, we leave the details to a forthcoming paper. $^{2}$

REMARK 6.9. Theorem 6.8 shows in particular that reversible solutions are stable with respect to smoothing of the coefficients and data by convolution with an averaging kernel.

\footnotetext{
${ }^{2}$ For convenience a proof can be found in the Appendix.
} 
By Oleinik's entropy condition (3.2) the case $\alpha(t)=C / t$ is of special interest if the initial data contain an up-jump. Then merely $\alpha \in L^{1}(] \tau, T[)$ for $\tau>0$ and Theorem 6.7 is only applicable on $] \tau, T\left[\times \mathbb{R}, \tau>0\right.$, instead of $\Omega_{T}$. For $\tau \rightarrow 0+$ this yields the definition of $p$ on the open set $\Omega_{T}$. Moreover, we see from (6.7)-(6.8) that $p \in H^{1,1}(] 0, T[\times]-R, R[) \cap L^{\infty}\left(\Omega_{T}\right) \cap$ $\left.\left.C^{0,1}(] 0, T\right] ; L_{l o c}^{1}(\mathbb{R})\right)$ for all $R>0$ and thus admits a $L^{1}$-trace $p(0, \cdot) \in L^{\infty}\left(\Omega_{T}\right)$ with $p(t, \cdot) \rightarrow p(0, \cdot)$ as $t \rightarrow 0+$ in $L_{l o c}^{r}(\mathbb{R})$ for all $r \in\left[1, \infty\left[\right.\right.$. Finally, $p \in L^{\infty}\left(0, T ; H_{l o c}^{1,1}(\mathbb{R})\right)$ and thus $p(0, \cdot) \in B V_{\text {loc }}(\mathbb{R})$. So we have the following extension of Theorem 6.8 .

THEOREM 6.10. Let the assumptions of Theorem 6.8 hold with the relaxation that $\alpha, \alpha_{n}$ are only uniformly bounded in $L^{1}(] \tau, T[)$ for each fixed $\tau>0$. Then there exist unique reversible solutions $\left.\left.p, p_{n} \in C_{l o c}^{0,1}\left(\Omega_{T}\right) \cap C^{0,1}(] 0, T\right] ; L_{\text {loc }}^{1}(\mathbb{R})\right)$ satisfying (6.6) for all $\left.s \in\right] 0, T[$. Moreover, (6.7)-(6.8) hold, $p, p_{n}$ are uniformly bounded in $\left.\left.H^{1,1}\left(\Omega_{T}\right) \cap C^{0,1}(] 0, T\right] ; L_{\text {loc }}^{1}(\mathbb{R})\right)$ and can thus be extended to $C^{0,1}\left([0, T] ; L_{\text {loc }}^{1}(\mathbb{R})\right)$. The traces $p(0, \cdot), p_{n}(0, \cdot)$ at $t=0$ are uniformly bounded in $L^{\infty}(\mathbb{R}) \cap B V_{\text {loc }}(\mathbb{R})$. Finally, $p_{n} \rightarrow p$ in $C_{l o c}\left(\Omega_{T}\right) \cap C\left([0, T] ; L_{\text {loc }}^{r}(\mathbb{R})\right)$ for all $r \in[1, \infty[$.

Proof. The statements are immediately clear by Theorems 6.7, 6.8, Remark 6.6 and the previous considerations. $\square$

It will be useful for our adjoint calculus to extend the definition of reversible solutions to the case where $p^{T} \in B(\mathbb{R})$ with $B(\mathbb{R})$ denoting the space of bounded functions. Clearly, Definition 6.5 makes also sense in this case. The following Theorem shows that the corresponding broad solutions are pointwise limits of Lipschitz backward solutions, if $p^{T}$ is the pointwise limit of Lipschitz functions.

THEOREM 6.11. Let $a, b$ be as in Theorem 6.10. Then for $p^{T} \in B(\mathbb{R})$ the corresponding reversible solution $p$ according to Definition 6.5 is unique, $p \in B\left(\Omega_{T}\right)$ and satisfies (6.7). If $p_{n}^{T} \in C^{0,1}(\mathbb{R})$ is a sequence that converges boundedly everywhere to $p^{T}$ then the corresponding reversible solutions $p^{n}$ according to Theorem 6.7 converge boundedly everywhere to $p$.

The proof follows easily by the definition of reversible solutions as broad solutions.

7. Proof of Lemma 5.2. We prove i) and will easily deduce ii). Throughout the proof we will use the notations of Lemma 5.1.

Let $\hat{u} \in \tilde{V}$ be fixed and $\tilde{u} \in \tilde{V}$ be arbitrary. In the following we write $\hat{y}$ and $\tilde{y}$ instead of $y(\cdot ; \hat{u})$ and $y(\cdot ; \tilde{u})$ and set $\Delta y \stackrel{\text { def }}{=} \tilde{y}-\hat{y}, \delta u=\tilde{u}-\hat{u}$.

For all sufficiently small $\varepsilon>0$ one has $\left.\hat{x}_{\mp} \stackrel{\text { def }}{=} x_{s}(\hat{u}) \mp \varepsilon \in\right] x_{-}, x_{+}\left[\right.$with $x_{\mp}$ as in Lemma 5.1. Our proof is based on the observation that

$$
\int_{\hat{x}_{-}}^{\hat{x}_{+}} \Delta y(\bar{t}, x) d x=\left(x_{s}(\tilde{u})-x_{s}(\hat{u})\right)\left[\hat{y}\left(\bar{t}, \hat{x}_{s}\right)\right]+O\left(\left(\varepsilon+\|\delta u\|_{\mathcal{V}}\right)\|\delta u\|_{\mathcal{V}}\right) .
$$

In fact, let $\hat{x}_{s}=x_{s}(\hat{u})$ and $x_{s, \mp} \stackrel{\text { def }}{=} \min / \max \left\{x_{s}(\hat{u}), x_{s}(\tilde{u})\right\}$, respectively. Since obviously $\left[\hat{y}\left(\bar{t}, \hat{x}_{s}\right)\right]=Y_{-}\left(\bar{t}, \hat{x}_{s}, \hat{u}\right)-Y_{+}\left(\bar{t}, \hat{x}_{s}, \hat{u}\right)$, we obtain by Lemma 5.1 ii)

$$
\begin{aligned}
& \int_{\hat{x}_{-}}^{\hat{x}_{+}} \Delta y(\bar{t}, x) d x-\left(x_{s}(\tilde{u})-x_{s}(\hat{u})\right)\left[\hat{y}\left(\bar{t}, \hat{x}_{s}\right)\right]= \\
& =\int_{\hat{x}_{s}-\varepsilon}^{x_{s,-}}\left(Y_{-}(\bar{t}, x, \tilde{u})-Y_{-}(\bar{t}, x, \hat{u})\right) d x+\int_{x_{s,+}}^{\hat{x}_{s}+\varepsilon}\left(Y_{+}(\bar{t}, x, \tilde{u})-Y_{+}(\bar{t}, x, \hat{u})\right) d x \\
& \quad+\int_{x_{s},-}^{\hat{x}_{s}}\left(Y_{-}\left(\bar{t}, \hat{x}_{s}, \hat{u}\right)-Y_{-}(\bar{t}, x, \hat{u})+Y_{+}(\bar{t}, x, \tilde{u})-Y_{+}\left(\bar{t}, \hat{x}_{s}, \hat{u}\right)\right) d x \\
& \quad+\int_{\hat{x}_{s}}^{x_{s,+}}\left(Y_{+}\left(\bar{t}, \hat{x}_{s}, \hat{u}\right)-Y_{+}(\bar{t}, x, \hat{u})+Y_{-}(\bar{t}, x, \tilde{u})-Y_{-}\left(\bar{t}, \hat{x}_{s}, \hat{u}\right)\right) d x \\
& \stackrel{\text { def }}{=} R_{1}+R_{2}+R_{3}+R_{4} .
\end{aligned}
$$


Using the Lipschitz continuity of $Y_{\mp}$ w.r.t. $\tilde{u}$, cf. Lemma 4.1, we get

$$
\left|R_{1}+R_{2}\right| \leq \varepsilon C\|\delta u\|_{\mathcal{V}}
$$

with $C$ independent of $\varepsilon$. Since $x_{s}$ in (5.1) is Lipschitz by Lemma 5.1 and there is by Lemma 4.1 also a uniform Lipschitz constant of $Y_{\mp}$ w.r.t. $x$ for all $\tilde{u} \in \tilde{V}$ we have

$$
\left|R_{3}+R_{4}\right| \leq C\|\delta u\|_{\mathcal{V}}^{2}
$$

with $C$ not depending on $\varepsilon$.

We now compute the left hand side of (7.1) by using an adjoint state. The difference of (1.1) for $\tilde{y}$ and $\hat{y}$ yields

$$
\partial_{t} \Delta y+\partial_{x}(f(\tilde{y})-f(\hat{y}))=g\left(t, x, \tilde{y}, \tilde{u}_{1}\right)-g\left(t, x, \hat{y}, \hat{u}_{1}\right) .
$$

We now define the functions

$$
\begin{gathered}
a \stackrel{\text { def }}{=} f^{\prime}(\hat{y}), \quad \tilde{a}(t, x) \stackrel{\text { def }}{=} \int_{0}^{1} f^{\prime}(\hat{y}(t, x)+\tau \Delta y(t, x)) d \tau \\
\tilde{b} \stackrel{\text { def }}{=} g_{y}\left(\cdot, \tilde{u}_{1}\right), \quad b \stackrel{\text { def }}{=} g_{y}\left(\cdot, \hat{u}_{1}\right) .
\end{gathered}
$$

Then by the definition of $\tilde{a}, \tilde{b}$ and since $g$ is affine linear in $y$ we can rewrite (7.2) as

$$
\partial_{t} \Delta y+\partial_{x}(\tilde{a} \Delta y)=\tilde{b} \Delta y+g\left(t, x, \hat{y}, \tilde{u}_{1}\right)-g\left(t, x, \hat{y}, \hat{u}_{1}\right) .
$$

Let $\tilde{p}$ be a test function satisfying for all $\tau>0, R>0$

$$
\tilde{p} \in C^{0,1}([\tau, \bar{t}] \times[-R, R]) \cap C\left([0, \bar{t}] ; L_{l o c}^{2}(\mathbb{R})\right) .
$$

Denote by $\hat{\zeta}_{\mp}$ the genuine backward characteristic of $\hat{y}=y(\cdot ; \hat{u})$ through $\left(\bar{t}, \hat{x}_{\mp}\right)$. Then by Lemma $5.1 \hat{\zeta}_{\mp}(0) \in J_{\mp}$ and $\hat{\zeta}_{-}(t) \leq \hat{\xi}_{-}(t) \leq \hat{\xi}_{+}(t) \leq \hat{\zeta}_{+}(t)$ for all $t \in[0, \bar{t}]$. By Lemma 4.4 there are $\delta>0$ and $\eta>0$ such that $\tilde{y}=Y_{\mp}(\cdot, \tilde{u})$ on $\left\{(t, x): t \in[0, \bar{t}],\left|x-\hat{\zeta}_{\mp}(t)\right|<\delta\right\}$ if $\|\delta u\|_{\tilde{\mathcal{V}}} \leq \eta$.

Now Oleinik's entropy condition (3.2) implies $\tilde{y}, \hat{y} \in B V([\tau, \bar{t}] \times[-R, R])$ for all $\tau>0$ and $R>0$. Hence, the same is true for $\Delta y, \tilde{a} \Delta y$. Therefore, if we multiply (7.5) by $\tilde{p}$ satisfying (7.6) and integrate over the domain between $\hat{\zeta}_{\mp}$ for $t \in[\tau, \bar{t}]$ we may apply integration by parts. Now $\Delta y(\tau, \cdot) \rightarrow \delta u_{0}$ and $\tilde{p}(\tau, \cdot) \rightarrow \tilde{p}(0, \cdot)$ in $L_{l o c}^{2}(\mathbb{R})$ as $\tau \rightarrow 0+$ by the initial condition and (7.6). Therefore, the integration by parts can be extended until $\tau=0$. Since $\frac{d}{d t} \hat{\zeta}_{\mp}(t)=f^{\prime}\left(\hat{y}\left(t, \hat{\zeta}_{\mp}(t)\right)\right)$ this gives

$$
\begin{aligned}
& \int_{\hat{x}_{-}}^{\hat{x}_{+}} \tilde{p}(\bar{t}, x) \Delta y(\bar{t}, x) d x= \\
& =\int_{\hat{\zeta}_{-}(0)}^{\hat{\zeta}_{+}(0)} \tilde{p}(0, x) \delta u_{0}(x) d x+\int_{0}^{\bar{t}} \int_{\hat{\zeta}_{-}(t)}^{\hat{\zeta}_{+}(t)} \Delta y\left(\partial_{t} \tilde{p}+\tilde{a} \partial_{x} \tilde{p}+\tilde{b} \tilde{p}\right) d x d t \\
& \quad+\int_{0}^{\bar{t}} \int_{\hat{\zeta}_{-}(t)}^{\hat{\zeta}_{+}(t)} \tilde{p}\left(g\left(t, x, \hat{y}, \tilde{u}_{1}\right)-g\left(t, x, \hat{y}, \hat{u}_{1}\right)\right) d x d t \\
& \quad+\int_{0}^{\bar{t}} \tilde{p}\left(t, \hat{\zeta}_{-}(t)\right)\left(-f^{\prime}(\hat{y}) \Delta y+f(\tilde{y})-f(\hat{y})\right)\left(t, \hat{\zeta}_{-}(t)\right) d t \\
& \quad+\int_{0}^{t} \tilde{p}\left(t, \hat{\zeta}_{+}(t)\right)\left(f^{\prime}(\hat{y}) \Delta y-f(\tilde{y})+f(\hat{y})\right)\left(t, \hat{\zeta}_{+}(t)\right) d t \\
& \stackrel{\text { def }}{=} I_{1}+I_{2}+I_{3}+I_{4}+I_{5} .
\end{aligned}
$$


Our aim is to choose $\tilde{p}$ as reversible solution of the adjoint equation

$$
\partial_{t} \tilde{p}+\tilde{a} \partial_{x} \tilde{p}=-\tilde{b} \tilde{p}, \quad \tilde{p}(\bar{t}, \cdot)=p^{\bar{t}} \equiv 1 /\left[y\left(\bar{t}, x_{s}(\hat{u}) ; \hat{u}\right)\right] .
$$

In the following, we justify that $\tilde{p}$ exists, satisfies (7.6) and study $\tilde{p}$ for $\tilde{u} \rightarrow \hat{u}$.

From the stability estimates of Theorem 3.1 we know that $\|\tilde{y}\|_{\infty} \leq M_{y}$ for all $\tilde{u} \in \tilde{V}$ and

$$
\|\Delta y\|_{1, l o c} \rightarrow 0 \quad \text { as }\|\delta u\|_{\tilde{\mathcal{V}}} \rightarrow 0
$$

Hence, we have $\tilde{a} \in L^{\infty}\left(\Omega_{\bar{t}}\right)$ uniformly bounded and $\tilde{a} \rightarrow f^{\prime}(\hat{y}) \stackrel{\text { def }}{=} a \in L^{\infty}\left(\Omega_{\bar{t}}\right)$ in $L_{l o c}^{1}\left(\Omega_{\bar{t}}\right)$ as $\|\delta u\|_{\tilde{\mathcal{V}}} \rightarrow 0$. Moreover, since $f^{\prime \prime}>0$ we get by Oleinik's entropy condition (3.2) that there exists a constant $C>0$ with

$$
\partial_{x} \tilde{a}(t, \cdot), \partial_{x} a(t, \cdot) \leq \frac{C}{t}
$$

By assumption, $\tilde{b}, b$ in (7.4) do not depend on $y$ and we have $\tilde{b}, b \in L^{\infty}\left(0, T ; C^{0,1}(\mathbb{R})\right)$ by (A1). Let $M_{u}>0$ such that $\left\|\tilde{u}_{1}\right\|_{\infty} \leq M_{u}$ for all $\tilde{u} \in \tilde{V}$. By (A2) $g_{y}$ admits a Lipschitz constant $L_{g^{\prime}}$ for $u_{1}$ on $\left[-M_{u}, M_{u}\right]^{m}$ and thus

$$
\|\tilde{b}-b\|_{L^{\infty}(0, T ; C(\mathbb{R}))} \leq L_{g^{\prime}}\left\|\delta u_{1}\right\|_{\infty} \leq L_{g^{\prime}}\|\delta u\|_{\tilde{\mathcal{V}}}
$$

Denoting by $\tilde{p}$ the reversible solution of (7.8) and by $p$ the reversible solution of (5.9) (i.e. of (7.8) with $a, b$ instead of $\tilde{a}, \tilde{b}$ ), we thus obtain from Theorem 6.10 that for all $\tau>0, R>0$

$$
\tilde{p}, p \in C^{0,1}([\tau, \bar{t}] \times[-R, R])
$$

and for all $r \in[1, \infty[$

$$
\tilde{p} \rightarrow p \text { in } C([\tau, \bar{t}] \times[-R, R]) \cap C\left([0, \bar{t}] ; L_{l o c}^{r}(\mathbb{R})\right) \quad \text { as }\|\delta u\|_{\tilde{\mathcal{V}}} \rightarrow 0 .
$$

We show that even

$$
\|\tilde{p}(0, \cdot)-p(0, \cdot)\|_{C(J)} \rightarrow 0 \quad \text { as }\|\delta u\|_{\tilde{\mathcal{V}}} \rightarrow 0
$$

In fact, since $u_{0}$ has only admissible discontinuities on $J_{a}$, there is by the definition of $\tilde{V}$ some $M_{0}>0$ with $\left.\partial_{x} \tilde{u}_{0}\right|_{J_{a}} \leq M_{0}$ in the sense of distributions for all $\tilde{u} \in \tilde{V}$. Since $J$ has its closure in $J_{a}$, there exists $\varepsilon>0$ such that $J_{2 \varepsilon} \subset J_{a}$ for the $2 \varepsilon$-neighborhood $J_{2 \varepsilon}$ of $J$. Moreover, $\|\tilde{a}\|_{\infty} \leq M_{a}$ for $M_{a}=\sup _{|y| \leq M_{y}}\left|f^{\prime}(y)\right|$ and all $\tilde{u} \in \tilde{V}$. Thus, there is $\tau_{0}>0$ such that

$$
K_{p} \stackrel{\text { def }}{=}\left\{(t, x) \in[0, \bar{t}] \times \mathbb{R}: \operatorname{dist}(x, J) \leq M_{a} t\right\} \subset\left([0, \tau] \times J_{\varepsilon}\right) \cup([\tau, \bar{t}] \times \mathbb{R})
$$

for all $\left.\tau \in] 0, \tau_{0}\right]$. The propagation speed of $\tilde{y}$ is uniformly bounded by $M_{a}$ for all $\tilde{u} \in \tilde{V}$. Hence, there is $\left.\tau \in] 0, \tau_{0}\right]$ such that $\left.\tilde{y}\right|_{[0, \tau] \times J_{\varepsilon}}$ does only depend on $\left(\left.\tilde{u}_{0}\right|_{J_{a}}, \tilde{u}_{1}\right)$. Smoothing $\tilde{u}_{0}$ outside $J_{a}$ thus yields by (3.2) that $\left.\partial_{x} \tilde{y}\right|_{[0, \tau] \times J_{\varepsilon}} \leq M_{1}$ for some $M_{1}>0$ and thus $\left.\partial_{x} \tilde{a}\right|_{[0, \tau] \times J_{\varepsilon}} \leq M_{2}$. Together with (7.9) we have possibly after increasing $M_{2}$

$$
\left.\partial_{x} \tilde{a}\right|_{K_{p}} \leq M_{2} .
$$

By Remark 6.6 $\left.\tilde{p}\right|_{K_{p}}$ depends only on the values of $\tilde{a}, \tilde{b}$ on $K_{p}$. Thus, by Theorem 6.8

$$
\tilde{p} \rightarrow p \text { in } C\left(K_{p}\right) \quad \text { as }\|\delta u\|_{\tilde{\mathcal{V}}} \rightarrow 0
$$

which implies (7.12). 
Now $\tilde{p}$ solves (7.8) a.e. by Theorem 6.10, which yields $I_{2}=0$. Moreover, (6.7) yields $M_{p}>0$ with $\|\tilde{p}\|_{\infty} \leq M_{p}$ for all $\tilde{u} \in \tilde{V}$ and we obtain by Lemma 4.1

$$
\left|I_{4 / 5}\right| \leq \int_{0}^{\bar{t}} M_{p} M_{f^{\prime \prime}}\left|\Delta y\left(t, \hat{\zeta}_{\mp}(t)\right)\right|^{2} d t \leq \bar{t} M_{p} M_{f^{\prime \prime}}\left\|Y_{\mp}(\cdot ; \tilde{u})-Y_{\mp}(\cdot ; \hat{u})\right\|_{\infty, S_{\mp}}^{2} \leq C\|\delta u\|_{\mathcal{V}}^{2}
$$

with $C$ independent of $\varepsilon$. Moreover, denoting the domain of integration by $D$ we have with (A2) and the dominated convergence Theorem

$$
\left\|g\left(\cdot, \hat{y}, \tilde{u}_{1}\right)-g\left(\cdot, \hat{y}, \hat{u}_{1}\right)-g_{u_{1}}\left(\cdot, \hat{y}, \hat{u}_{1}\right) \delta u_{1}\right\|_{1, D}=o\left(\left\|\delta u_{1}\right\|_{\infty}\right)
$$

independently of $\varepsilon$. Therefore,

$$
\left|I_{3}-\int_{0}^{\bar{t}} \int_{\hat{\zeta}_{-}(t)}^{\hat{\zeta}_{+}(t)} p g_{u_{1}}\left(t, x, \hat{y}, \hat{u}_{1}\right) \delta u_{1} d x d t\right| \leq\|p\|_{\infty} o\left(\left\|\delta u_{1}\right\|_{\infty}\right)+C\|\tilde{p}-p\|_{1, D}\left\|\delta u_{1}\right\|_{\infty}
$$

with $C$ independent of $\varepsilon$. From the backward stability of genuine backward characteristics we find $L_{\zeta}>0$ independent of $\varepsilon$ with $\left|\hat{\zeta}_{\mp}(t)-\hat{\xi}_{\mp}(t)\right| \leq L_{\zeta} \varepsilon$ for all $t \in[0, \bar{t}]$. Thus, we obtain with the last inequality

$$
\left|I_{3}-\int_{0}^{\bar{t}} \int_{\hat{\xi}_{-}(t)}^{\hat{\xi}_{+}(t)} p g_{u_{1}}\left(t, x, \hat{y}, \hat{u}_{1}\right) \delta u_{1} d x d t\right| \leq C\left(\varepsilon+\|\tilde{p}-p\|_{1, D}\right)\|\delta u\|_{\mathcal{V}}+o\left(\|\delta u\|_{\mathcal{V}}\right)
$$

for some $C>0$ independent of $\varepsilon$. Moreover, since $\hat{\xi}_{\mp}(0), \hat{\zeta}_{\mp}(0) \in J_{\mp}$

$$
\begin{aligned}
\left|I_{1}-\int_{\hat{\xi}_{-}(0)}^{\hat{\xi}_{+}(0)} p \delta u_{0} d x\right| \leq & \left(L_{\zeta} \varepsilon\|\tilde{p}(0, \cdot)\|_{\infty}+\|(\tilde{p}-p)(0, \cdot)\|_{1, J_{r} \cup J_{-} \cup J_{+}}\right)\left\|\delta u_{0}\right\|_{\infty, J_{r} \cup J_{-} \cup J_{+}} \\
& +\|(\tilde{p}-p)(0, \cdot)\|_{\infty, J}\left\|\delta u_{0}\right\|_{1, J} \\
\leq & C\left(\varepsilon+\|(\tilde{p}-p)(0, \cdot)\|_{1, J_{r} \cup J_{-} \cup J_{+}}+\|(\tilde{p}-p)(0, \cdot)\|_{\infty, J}\right)\|\delta u\|_{\tilde{\mathcal{V}}} .
\end{aligned}
$$

Using the estimates for $I_{1}, \ldots, I_{5}, R_{1}, \ldots, R_{4}$ together with (7.11), (7.12) the Fréchet differentiability of $x_{s}:\left(\tilde{V},\|\cdot\|_{\tilde{\mathcal{V}}}\right) \longrightarrow \mathbb{R}$ and the formula for the derivative are obvious. The derivative is also continuous. In fact, denote by $\tilde{p}$ now the reversible solution of (7.8) with $\tilde{p}^{\bar{t}}=1 /\left[\tilde{y}\left(\bar{t}, x_{s}(\tilde{u})\right)\right], \tilde{a}=f^{\prime}(\tilde{y}), \tilde{b}=g_{y}\left(\cdot, \tilde{u}_{1}\right)$. Then we already know that $\tilde{p}^{\bar{t}} \rightarrow p^{\bar{t}}$ in $C(\mathbb{R})$ as $\|\delta u\|_{\tilde{\mathcal{V}}} \rightarrow 0$ and $\tilde{a}, \tilde{b}$ have the same properties as above. Thus, we obtain again that (7.11), (7.12) hold. Together with the Lipschitz continuity of $\hat{u} \in\left(\tilde{V},\|\cdot\|_{\tilde{\mathcal{V}}}\right) \longmapsto \hat{\xi}_{\mp}(\cdot) \in C([0, T])$ and the definition of $\|\cdot\|_{\tilde{\mathcal{V}}}$ this shows immediately that the derivative of $x_{s}$ is continuous.

ii): In this case the adjoint equation has no source term and has thus the constant reversible solution $\hat{p}=p \equiv 1 /\left[y\left(\bar{t}, x_{s}(\hat{u}) ; \hat{u}\right)\right]$. Taking $\left(\tilde{V},\|\cdot\|_{\tilde{\mathcal{V}}}\right)$ instead of $\left(\hat{V},\|\cdot\|_{\mathcal{V}}\right)$ was only necessary to ensure (7.10)-(7.12), which are now trivial. The proof is now exactly as before, but less technical since $\tilde{p}=p=$ const.

8. Differentiability in continuity points of class $\mathbf{C B}^{c}$. Now we consider the situation where additional discontinuities are created in the initial data. More precisely, let $(\bar{t}, \bar{x})$ be a continuity point of $y(\cdot ; u)$ of class $\mathrm{CB}^{c}$, i.e. outside of the shock set such that the backward characteristic $\xi$ through $(\bar{t}, \bar{x})$ meets $t=0$ in a continuity point $\bar{z}$ of $u_{0}$. If $\bar{z}=x_{i}$ for some $i \in\{1, \ldots, N\}$ then $u_{0}$ is not necessarily differentiable at $\bar{z}$ and a shift variation of $u_{0}$ can create a discontinuity at $x_{i}$, since it allows locally a variation in $P C^{1}\left(J ; x_{i}\right)$ with an interval $J$ containing $x_{i}$. 
We have already observed in $\S 3.3$ that the one-sided derivatives in $\bar{z}$ satisfy (3.17). Thus, if we define the $C^{1}$-prolongations

$$
\left.u_{0}^{\mp} \stackrel{\text { def }}{=} u_{0}\right|_{\{\mp(z-\bar{z}) \geq 0\}}+\left.\left(u_{0}(\bar{z} \mp)+u_{0}^{\prime}(\bar{z} \mp)(.-\bar{z})\right)\right|_{\{\mp(z-\bar{z})<0\}} .
$$

then there are $z_{-}<\bar{z}<z_{+}, \rho, \beta>0$ such that (4.1) holds for $u_{0}^{\mp}$ instead of $u_{0}$. Thus, Lemma 4.1 is applicable for $u_{0}^{\mp}$ instead of $u_{0}$ yielding $\left(Y_{\mp}, Z_{\mp}, V_{\mp}, S_{\mp}(\tau), J\right)=\operatorname{YC}\left(u_{0}^{\mp}, u_{1},\left[z_{-}, z_{+}\right]\right)$. Now it is obvious, that for $\delta>0$ small enough and

$$
V=\left\{\hat{u}=\left(\hat{u}_{0}, \hat{u}_{1}\right) \in \mathcal{V}:\|\hat{u}-u\|_{\mathcal{V}}<\delta\right\}, \quad \mathcal{V} \stackrel{\text { def }}{=} P C^{1}(J ; \bar{z}) \times L^{\infty}\left(0, T ; C^{1}(\mathbb{R})^{m}\right)
$$

the mappings

$$
\hat{u} \in V \longmapsto\left(Z_{\mp}, Y_{\mp}\right)\left(\cdot, \hat{u}_{0}^{\mp}, \hat{u}_{1}\right) \in C\left(S_{\mp}\right) .
$$

are continuously differentiable. The next Lemma shows that piecing together $Y_{\mp}$ along $\xi$ yields a first order approximation of $y(\cdot, \hat{u})$ in $u$ for $\hat{u} \in V$.

LEMMA 8.1. Let $(\bar{t}, \bar{x})$ be a continuity point of $y(\cdot ; u)$ outside of the shock set such that the backward characteristic $\xi$ through $(\bar{t}, \bar{x})$ meets $t=0$ in a continuity point $\bar{z}$ of $u_{0}$. Let $z_{-}<\bar{z}<z_{+},\left(Y_{\mp}, Z_{\mp}, V_{\mp}, S_{\mp}(\tau), J\right)=\mathrm{YC}\left(u_{0}^{\mp}, u_{1},\left[z_{-}, z_{+}\right]\right)$and $V, \mathcal{V}$ be defined as in (8.2) for $\delta>0$ small enough. Then the mapping

$$
\begin{aligned}
& \hat{u} \in V \longmapsto \tilde{y}(\cdot ; \hat{u}) \in C\left(S_{-} \cap\{x \leq \xi(t)\}\right) \cap C\left(S_{+} \cap\{x>\xi(t)\}\right) \\
& \tilde{y}(t, x ; \hat{u}) \stackrel{\text { def }}{=} \begin{cases}Y_{-}\left(t, x,\left.\hat{u}_{0}^{-}\right|_{J}, \hat{u}_{1}\right), & (t, x) \in S_{-} \cap\{x \leq \xi(t)\} \\
Y_{+}\left(t, x,\left.\hat{u}_{0}^{+}\right|_{J}, \hat{u}_{1}\right), & (t, x) \in S_{+} \cap\{x>\xi(t)\}\end{cases}
\end{aligned}
$$

is continuously Fréchet differentiable.

For any $M_{\infty}>0$ there are $R>0, \nu>0$ and a neighborhood $\left.\hat{I}=\right] x_{-}, x_{+}[$of $\bar{x}$ such that with

$$
\begin{aligned}
\hat{V} \stackrel{\text { def }}{=}\left\{\left(\hat{u}_{0}, \hat{u}_{1}\right) \in L^{\infty}(\mathbb{R}) \times\right. & L^{\infty}\left(0, T ; C^{1}(\mathbb{R})^{m}\right):\left(\left.\hat{u}_{0}\right|_{J}, \hat{u}_{1}\right) \in V, \\
& \left.\left\|\hat{u}_{0}-u_{0}\right\|_{\infty, \mathbb{R} \backslash J} \leq M_{\infty},\left\|\hat{u}_{0}-u_{0}\right\|_{1,[-R, R] \backslash J} \leq \nu\right\} .
\end{aligned}
$$

for all $r \in[1, \infty[$ holds

$$
\lim _{\substack{\hat{u} \in \hat{V} \\\|\hat{u}-u\|_{\mathcal{V}} \rightarrow 0}} \frac{\|y(\bar{t}, \cdot ; \hat{u})-\tilde{y}(\bar{t}, \cdot ; \hat{u})\|_{r, \hat{I}}}{\|\hat{u}-u\|_{\mathcal{V}}}=0 .
$$

In particular, $\hat{u} \in\left(\hat{V},\|\cdot\|_{\mathcal{V}}\right) \longmapsto y(\bar{t}, \cdot ; \hat{u}) \in L^{r}(\hat{I})$ is Fréchet differentiable at $u$.

Proof. The construction of $J, V, Z_{\mp}$ and $Y_{\mp}$ was already justified above. Set $J_{\mp}=$ $J \cap\{\mp(z-\bar{z})>0\}$. Moreover, since (8.3) are continuously Fréchet differentiable the same is true for (8.4). Since $(\bar{t}, x)$ is a continuity point outside the shock set we know that (4.1) holds on an interval $J$ containing $\bar{z}$ for $u_{0}$ (and also for $u_{0}^{\mp}$ instead of $u_{0}$ ) and we obtain as at the beginning of the proof of Lemma $4.4 x_{-}<\bar{x}<x_{+}$such that

$$
y(\bar{t}, x ; u)=\tilde{y}(\bar{t}, x ; u) \quad \forall x \in \hat{I} \stackrel{\text { def }}{=}] x_{-}, x_{+}[.
$$

Given $M_{\infty}>0$ and $\varepsilon>0$ we thus find by 4.4 ii) $R>0$ and $\nu>0$ such that after a possible reduction of $\delta$ (and hence $V$ ) holds

$$
y(\bar{t}, x ; \hat{u})=\tilde{y}(\bar{t}, x ; \hat{u}) \quad \forall x \in \hat{I} \backslash[\bar{x}-\varepsilon / 2, \bar{x}+\varepsilon / 2], \quad \forall \hat{u} \in \hat{V} .
$$

Hereby we have used, that $\left.\hat{u}_{0}\right|_{J_{\mp}}=\left.\hat{u}_{0}^{\mp}\right|_{J_{\mp}}$. One easily sees that $R>0$ and $\nu>0$ can be chosen independent from $\varepsilon>0$. For $\varepsilon>0$ small enough the genuine backward characteristics 
$\hat{\xi}_{\mp}$ of $y(\cdot ; \hat{u})$ through $(\bar{t}, \bar{x} \mp \varepsilon)$ satisfy $\hat{\xi}_{\mp}(0)=Z_{\mp}(\bar{t}, \bar{x} \mp \varepsilon, \hat{u}) \in J$ and thus for all genuine backward characteristics $\xi$ through $(\bar{t}, x),|x-\bar{x}| \leq \varepsilon$ holds $\xi(0) \in J$. Let $x$ be an arbitrary continuity point with $|x-\bar{x}| \leq \varepsilon$ and set $\xi(0)=z$. Then clearly

$$
\begin{aligned}
|y(\bar{t}, x ; \hat{u})-y(\bar{t}, x ; u)| \leq & \left|v\left(\bar{t} ; z, \hat{u}_{0}(z), \hat{u}_{1}\right)-v\left(\bar{t} ; z, u_{0}(z), u_{1}\right)\right| \\
& +L_{x}\left|\zeta\left(\bar{t} ; z, \hat{u}_{0}(z), \hat{u}_{1}\right)-\zeta\left(\bar{t} ; z, u_{0}(z), u_{1}\right)\right| \\
\leq & \left(1+L_{x}\right) L\left(\left|\hat{u}_{0}(z)-u_{0}(z)\right|+\left\|\hat{u}_{1}-u_{1}\right\|_{L^{\infty}(0, T ; C(\mathbb{R}))}\right) .
\end{aligned}
$$

where $L_{x}$ is a Lipschitz constant of $y(\bar{t}, \cdot ; u)$ on $\hat{I}$ and $L$ is a Lipschitz constant of $v$, cf. Lemma 3.3. Moreover, using the local Lipschitz continuity of (8.3) we obtain with a constant $L_{Y}>0$

$$
|\tilde{y}(\bar{t}, x ; \hat{u})-y(\bar{t}, x ; u)| \leq \max \left(\left|Y_{\mp}\left(\bar{t}, x, \hat{u}_{0}^{\mp}, \hat{u}_{1}\right)-Y_{\mp}\left(\bar{t}, x, u_{0}^{\mp}, u_{1}\right)\right|\right) \leq L_{Y}\|\hat{u}-u\|_{\mathcal{V}} .
$$

This together gives for $r \in[1, \infty[$

$$
\|y(\bar{t}, \cdot ; \hat{u})-\tilde{y}(\bar{t}, \cdot ; \hat{u})\|_{r, \hat{I}} \leq C \varepsilon^{1 / r}\|\hat{u}-u\|_{\mathcal{V}}
$$

with $C>0$ independent of $\hat{u} \in \hat{V}$ and $\varepsilon$. The proof is complete by letting $\varepsilon$ tend to zero.

9. Differentiability on the boundary of rarefaction waves (case $\mathbf{R B}^{c}$ ). In a last step we consider continuity points $(\bar{t}, \bar{x})$ of class $\mathrm{RB}^{c}$, i.e. outside the shock set and on the boundary of a rarefaction wave. If $(\bar{t}, \bar{x})$ is for concreteness on the left boundary of a rarefaction wave then the backward characteristic meets $t=0$ in a discontinuity $\bar{z}=x_{i}$ of $u_{0}$ with $u_{0}\left(x_{i}-\right)<u_{0}\left(x_{i}+\right)$ and (3.9), (3.10) hold with $\bar{w}=u_{0}\left(x_{i}-\right)$. Finally (3.17) holds for the left sided, (3.20) for the right sided derivative, respectively. Hence, we get $\left(Y_{r}, W, V_{1}, S_{+}(\tau), J_{w}\right)=\operatorname{YR}\left(u, \bar{t}, \bar{z},\left[w_{-}, w_{+}\right]\right)$by Lemma 4.5 for suitable $w_{-}<\bar{w}<w_{+}$ and $\left(Y_{-}, Z_{-}, V_{-}, S_{-}(\tau), J\right)=\mathrm{YC}\left(u_{0}^{-}, u_{1},\left[z_{-}, z_{+}\right]\right)$by Lemma 4.1 for suitable $z_{-}<\bar{z}<z_{+}$, where $u_{0}^{-}$is the $C^{1}$-prolongation (8.1). For $V$ according to (8.2) with $\delta>0$ small enough we can achieve that $\hat{u} \in V$ implies $\hat{u}_{1} \in V_{1}$ and $\left(\hat{u}_{0}^{-}, \hat{u}_{1}\right) \in V_{-}$. Now it is obvious that $Y_{-}(\cdot, \hat{u})$ and $Y_{r}\left(\cdot, \hat{u}_{1}\right)$ can be glued together continuously along the forward characteristic $\hat{\xi}(t) \stackrel{\text { def }}{=} \zeta\left(t ; \bar{z}, \hat{u}_{0}(\bar{z}-), \hat{u}_{1}\right)$, for all $\hat{u} \in V$ yielding a classical solution on $\left(S_{-} \cap\{x \leq\right.$ $\hat{\xi}(t)\}) \cup\left(S_{+} \cap\{x>\hat{\xi}(t)\}\right)$. The next Lemma shows that this function coincides locally with $y(\cdot ; \hat{u})$.

Lemma 9.1. Let $(\bar{t}, \bar{x})$ be a continuity point of $y(\cdot ; u)$ outside the shock set on the boundary of a rarefaction wave, i.e. the backward characteristic meets $t=0$ in $\bar{z}=x_{i}$ with $u_{0}(\bar{z}-)<u_{0}(\bar{z}+)$ and (3.9), (3.10) hold with $\bar{w}=u_{0}(\bar{z}-)$ or $\bar{w}=u_{0}(\bar{z}+)$.

Given $M_{\infty}>0$ there are $\delta>0, R>0$ and $\nu>0$ such that with $V$ according to (8.2) and $\tilde{V}$ as in Lemma 8.1 the following holds:

$\hat{\xi} \stackrel{\text { def }}{=} \zeta\left(\cdot ; \bar{z}, \hat{u}(\bar{z}-), \hat{u}_{1}\right)$ is on $[0, \bar{t}]$ for all $\hat{u} \in \hat{V}$ a genuine forward characteristic of $y(\cdot ; \hat{u})$ through $(0, \bar{z})$. Furthermore:

i) If $\bar{w}=u_{0}(\bar{z}-)$ then let $\left(Y_{-}, Z_{-}, V_{-}, S_{-}(\tau), J\right)=\mathrm{YC}\left(u_{0}^{-}, u_{1},\left[z_{-}, z_{+}\right]\right)$as well as $\left(Y_{r}, W, V_{1}, S_{+}(\tau), J_{w}\right)=\operatorname{YR}\left(u, \bar{t}, \bar{z},\left[w_{-}, w_{+}\right]\right)$be defined as explained above. Then there is a neighborhood $\hat{I}=] x_{-}, x_{+}[$of $\bar{x}$ such that for all $\hat{u} \in \hat{V}$ holds

$$
y(\bar{t}, x ; \hat{u}) \stackrel{\text { def }}{=} \begin{cases}Y_{-}\left(\bar{t}, x, \hat{u}_{0}^{-}, \hat{u}_{1}\right), & x \in] x_{-}, \hat{\xi}(\bar{t})[ \\ Y_{r}\left(\bar{t}, x, \hat{u}_{1}\right), & x \in] \hat{\xi}(\bar{t}), x_{+}[\end{cases}
$$

If $\hat{V}$ is equipped with the norm

$$
\left\|\left(\hat{u}_{0}, \hat{u}_{1}\right)\right\|_{\mathcal{V}} \stackrel{\text { def }}{=}\left\|\hat{u}_{0}\right\|_{C^{1}\left(J_{-}\right)}+\left\|\hat{u}_{1}\right\|_{L^{\infty}\left(0, T ; C^{1}(\mathbb{R})\right)},
$$

where $J_{-} \stackrel{\text { def }}{=} J \cap\{z<\bar{z}\}$ then

$$
\hat{u} \in\left(\hat{V},\|\cdot\|_{\mathcal{V}}\right) \longmapsto y(\bar{t}, \cdot ; \hat{u}) \in L^{r}(\hat{I})
$$


is continuously Fréchet differentiable for all $r \in[1, \infty[$ with derivative

$$
\begin{aligned}
d_{u} y(\bar{t}, \cdot ; \hat{u}) \cdot\left(\delta u_{0}, \delta u_{1}\right)= & \mathbf{1}_{] x_{-}, \hat{\xi}(\bar{t})[}\left(d_{u} Y_{-}\left(\bar{t}, \cdot, \hat{u}_{0}^{-}, \hat{u}_{1}\right) \cdot\left(\left.\delta u_{0}\right|_{J_{-}}, \delta u_{1}\right)\right) \\
& +\mathbf{1}_{] \hat{\xi}(\bar{t}), x_{+}[}\left(d_{u_{1}} Y_{r}\left(\bar{t}, \cdot, \hat{u}_{1}\right) \cdot \delta u_{1}\right) .
\end{aligned}
$$

ii) If $\bar{w}=u_{0}(\bar{z}+)$ a completely analogous result holds with left state $Y_{r}$ and right state $Y_{+}$ obtained from $\left(Y_{+}, Z_{+}, V_{+}, S_{+}(\tau), J\right)=\mathrm{YC}\left(u_{0}^{+}, u_{1},\left[z_{-}, z_{+}\right]\right)$.

Proof. We only sketch the proof, since it is similar to the proof of Lemma 8.1. First, we obtain that (9.1) holds for $u$ and an appropriate neighborhood $\hat{I}$ of $\bar{x}$. For given $M_{\infty}>0$ and $\varepsilon>0$ we conclude by applying Lemmas 4.4, 4.8 that (9.1) holds also on $\hat{I} \cap\{|x-\bar{x}|>\varepsilon / 2\}$ for all $\hat{u} \in \hat{V}$ with $\hat{V}$ as in Lemma 8.1 and $V$ as in (8.2). Hence, $y(\bar{t}, x ; \hat{u})$ depends for $x \in[\bar{x}-\varepsilon, \bar{x}+\varepsilon]$ only on the values of $\hat{u}_{0}$ on $J_{-}$. But glueing together $Y_{-}(\cdot, \hat{u})$ and $Y_{r}\left(\cdot, \hat{u}_{1}\right)$ along $\hat{\xi}$ yields a classical solution that is compatible with the initial data $\left.\hat{u}_{0}\right|_{J_{-}}$and coincides with $y(\cdot ; \hat{u})$ along all genuine backward characteristics through $(\bar{t}, x), x \in \hat{I} \backslash[\bar{x}-\varepsilon, \bar{x}+\varepsilon]$. Hence, (9.1) holds by the uniqueness of entropy solutions.

From (9.1) the continuous differentiability of (9.2) is obvious, since by Lemmas 4.1 and 4.5 the mappings $\hat{u} \in\left(\hat{V},\|\cdot\|_{\mathcal{V}}\right) \longmapsto\left(Y_{-}\left(\bar{t}, \cdot ; \hat{u}_{0}^{-}, \hat{u}_{1}\right), Y_{r}\left(\bar{t}, \cdot ; \hat{u}_{1}\right)\right) \in C(\hat{I})^{2}$ are continuously Fréchet differentiable, the functions on the right remain bounded in $C^{1}(\hat{I})$ on $\hat{V}$ and $\hat{\xi}(\bar{t})$ depends Lipschitz continuously on $\hat{u}$. The formula for the derivative is obvious.

10. Shift-differentiability and a sensitivity calculus for entropy solutions. In this section we will use the results of the previous sections to prove the shift-differentiability result for entropy solutions already outlined in the introduction. As a consequence we obtain a sensitivity calculus which is based on shift-variations. As in Lemma 5.2 we make the additional assumption

(A4) $g$ is affine linear w.r.t. $y$.

Then we obtain the following evolution result for shift-variations:

TheOREM 10.1. Let (A1)-(A4) hold. Let $u_{0} \in P C^{1}\left(\mathbb{R} ; x_{1}, \ldots, x_{N}\right)$ with $x_{1}<$ $\ldots<x_{N}$, and let $u_{1} \in L^{\infty}\left(0, T ; C^{1}(\mathbb{R})^{m}\right)$. For $u=\left(u_{0}, u_{1}\right)$ let $y(u) \in L^{\infty}\left(\Omega_{T}\right) \cap$ $C\left([0, T] ; L_{l o c}^{1}(\mathbb{R})\right)$ be the entropy solution of $(1.1)$. Finally, let $I=[a, b], a<b$, and $\left.\left.\bar{t} \in\right] 0, T\right]$ such that $y(\bar{t}, \cdot ; u)$ has no shock generation points on I and finitely many shocks on $I$ at $a<\bar{x}_{1}<\ldots<\bar{x}_{K}<b$ that are no shock interaction points.

Let $W \stackrel{\text { def }}{=} P C^{1}\left(\mathbb{R} ; x_{1}, \ldots, x_{N}\right) \times L^{\infty}\left(0, T ; C^{1}(\mathbb{R})^{m}\right) \times \mathbb{R}^{N}$ be equipped with the norm

$$
\left\|\left(w_{0}, w_{1}, \sigma\right)\right\|_{W}=\left\|w_{0}\right\|_{P C^{1}\left(\mathbb{R} ; x_{1}, \ldots, x_{N}\right)}+\left\|w_{1}\right\|_{L^{\infty}\left(0, \bar{t} ; C^{1}(\mathbb{R})\right)}+\|\sigma\|_{2} .
$$

Then the mapping

$$
\left.\left(w_{0}, w_{1}, \sigma\right) \in W \longmapsto y\left(\bar{t}, \cdot ; u_{0}+S_{u_{0}}^{\left(x_{i}\right)}\left(w_{0}, \sigma\right), u_{1}+w_{1}\right)\right|_{I}
$$

is shift-differentiable w.r.t. $\left(w_{0}, w_{1}, \sigma\right)$ at 0 and the shift-derivative $T_{s}(0)=D_{s} y(\bar{t} ; u)$ satisfies $T_{s}(0) \in \mathcal{L}\left(W, P C\left(I ; \tilde{x}_{1}, \ldots, \tilde{x}_{\tilde{K}}\right) \times \mathbb{R}^{K}\right)$, where $\tilde{x}_{1}<\ldots<\tilde{x}_{\tilde{K}}$ contain in addition to $\bar{x}_{1}, \ldots, \bar{x}_{K}$ all points in I of type $C B^{c}$.

If all $x_{i}, i=1, \ldots, N$, are discontinuities of $u_{0}$, i.e. $u_{0}\left(x_{i}-\right) \neq u_{0}\left(x_{i}+\right)$, then (10.1) is continuously shift-differentiable on $\left\{\|(w, \sigma)\|_{W}<\rho\right\}$ for $\rho>0$ sufficiently small and $T_{s}(0) \in \mathcal{L}\left(W, P C\left(I ; \bar{x}_{1}, \ldots, \bar{x}_{K}\right) \times \mathbb{R}^{K}\right)$.

Formula for the shift-derivative. The shift derivative $(\delta y, \bar{s})=T_{s}(0) \cdot\left(\delta w_{0}, \delta w_{1}, s\right)$ is given as follows: With $\bar{x}_{0} \stackrel{\text { def }}{=} a, \bar{x}_{K+1} \stackrel{\text { def }}{=}$, denote by $\xi_{k}^{\mp}$ the minimal/maximal backward characteristic through $\left(\bar{t}, \bar{x}_{k}\right), k=0, \ldots, K+1$, by $S_{k}$ the domain confined by $\xi_{k}^{+}$and $\xi_{k+1}^{-}, k=0, \ldots, K$, by $D_{k}$ the domain confined by $\xi_{k}^{-}$and $\xi_{k}^{+}, k=1, \ldots, K$, and set $I_{k}=D_{k}^{c l} \cap\{t=0\}$. Let $\delta Y$ on all $S_{k}$ be the broad solution of the linearized equation

$$
\partial_{t} \delta Y+\partial_{x}\left(f^{\prime}(y) \delta Y\right)=g_{y}\left(t, x, y, u_{1}\right) \delta Y+g_{u_{1}}\left(t, x, y, u_{1}\right) \delta u_{1}
$$


with initial conditions

$$
\delta Y=\delta w_{0} \text { on } S_{k}^{c l} \backslash\left\{x_{i}\right\}, \quad \delta Y=0 \text { on } S_{k}^{c l} \cap\left\{x_{i}\right\}
$$

according to Remarks 4.3 and 4.7, respectively. Moreover, let $p$ on each $D_{k}$ be the reversible solution of the adjoint equation

$$
\partial_{t} p+f^{\prime}(y) \partial_{x} p=-g_{y}\left(t, x, y, u_{1}\right) p, \quad p(\bar{t}, .)=1 /\left[y\left(\bar{t}, \bar{x}_{k} ; u\right)\right] .
$$

Then $(\delta y, \bar{s})=T_{s}(0) \cdot\left(\delta w_{0}, \delta w_{1}, s\right)$ is given by

$$
\begin{aligned}
\delta y & =\delta Y(\bar{t}, \cdot) \\
\bar{s}_{k} & =\left(p(0, \cdot), \delta w_{0}\right)_{2, I_{k}}+\sum_{x_{i} \in I_{k}} p\left(0, x_{i}\right)\left[u_{0}\left(x_{i}\right)\right]_{+} s_{i}+\left(p g_{u_{1}}\left(t, x, y, u_{1}\right), \delta w_{1}\right)_{2, D_{k}}
\end{aligned}
$$

Proof. The proof has three parts. We consider first shock points, then continuity points.

Step 1: We show first the shift-differentiability in a neighborhood of a shock point. Set $y=y(\cdot ; u)$. By assumption, $y(\bar{t}, \cdot)$ contains on $I$ finitely many shocks at $\bar{x}_{1}, \ldots, \bar{x}_{K}$ satisfying the assumptions of Lemma 5.1 or Corollary 5.3. Let us for concreteness assume that $\bar{x}_{k}$ is of type $\mathrm{C}^{c} \mathrm{C}^{c}$, i.e. satisfies the scenario of Lemma 5.1. The case where left and/or right state lie on a rarefaction wave can be treated very similar using Corollary 5.3.

Thus, for $\bar{x}=\bar{x}_{k}$ Lemmas 5.1 and 5.2 are applicable. We verify that with the notations of Lemma 5.1 for $\|(w, \sigma)\|_{W}<\rho, \rho>0$ sufficiently small, holds

$$
u^{s} \stackrel{\text { def }}{=}\left(u_{0}+w_{0}^{s}, u_{1}+w_{1}\right) \in \hat{V},\left\|\left(w_{0}^{s}, w_{1}\right)\right\|_{\mathcal{V}} \leq C\|(w, \sigma)\|_{W} \text { for } w_{0}^{s}=S_{u_{0}}^{\left(x_{i}\right)}\left(w_{0}, \sigma\right) .
$$

In fact, there is $\rho>0$ with $\left.w_{0}^{s}\right|_{J_{\mp}}=\left.w_{0}\right|_{J_{\mp}}$ whenever $\|\sigma\|_{2}<\rho$. Moreover, for all $R>0$

$$
\left\|S_{u_{0}}^{\left(x_{i}\right)}\left(w_{0}, \sigma\right)\right\|_{1,[-R, R]} \leq 2 R\left\|w_{0}\right\|_{\infty}+2\|y(\bar{t}, \cdot ; u)\|_{\infty}\|\sigma\|_{1} \leq C\|(w, \sigma)\|_{W} .
$$

Hence, for sufficiently small $\rho>0$ (10.7) is obvious and Lemma 5.1 as well as i) of Lemma 5.2 hold with $\hat{u}=u^{s}$ in (10.7) if $\|(w, \sigma)\|_{W}<\rho$. As a consequence, $y\left(\bar{t}, \cdot ; u^{s}\right)$ has in a neighborhood $\hat{I}=] x_{-}, x_{+}\left[\right.$of $\bar{x}$ the form (5.2) and the shock position $x_{s}\left(u^{s}\right)$ in (5.1) depends by (10.7) and Lemma 5.1 ii) Lipschitz continuously on $(w, \sigma) \in W$. To cover also i) of Lemma 5.2 we choose the open neighborhood $J \subset J_{a}$ in i) such that it contains all admissible discontinuities of $u_{0}$ between $J_{-}$and $J_{+}$. Then (10.7) holds with $\tilde{V}, \tilde{\mathcal{V}}$ instead of $\hat{V}, \mathcal{V}$ and for $w_{0}^{s}=S_{u_{0}}^{\left(x_{i}\right)}\left(w_{0}, \sigma\right)$ replaced by $\tilde{w}_{0}^{s} \stackrel{\text { def }}{=} S_{u_{0}+w_{0}}^{\left(x_{i}\right)}\left(w_{0}, \sigma\right)$. In fact, since $S_{u_{0}+w_{0}}^{\left(x_{i}\right)}\left(w_{0}, \sigma\right)$ shifts only admissible discontinuities without creating additional ones, there are $M_{L}>0, \rho>0$ such that $\|(w, \sigma)\|_{W}<\rho$ implies $\left.\tilde{w}_{0}^{s}\right|_{J_{\mp} \cup J_{r}}=\left.w_{0}\right|_{J_{\mp} \cup J_{r}}$ and $\left.\partial_{x}\left(u_{0}+\tilde{w}_{0}^{s}\right)\right|_{J_{a}} \leq M_{L}$.

We show that $(w, \sigma) \in W \longmapsto x_{s}\left(u^{s}\right)$ is Fréchet differentiable at 0 . Consider $(\delta w, s) \in W$ with $\|(\delta w, s)\|_{W}<\rho$ and set $\hat{u}=\left(u_{0}+\delta w_{0}^{s}, u_{1}+\delta w_{1}\right), \tilde{u}=\left(u_{0}+\delta \tilde{w}_{0}^{s}, u_{1}+\delta w_{1}\right)$ with

$$
\delta w_{0}^{s}=S_{u_{0}}^{\left(x_{i}\right)}\left(\delta w_{0}, s\right), \quad \delta \tilde{w}_{0}^{s}=S_{u_{0}+\delta w_{0}}^{\left(x_{i}\right)}\left(\delta w_{0}, s\right),
$$

Then for $\|(\delta w, s)\|_{W}<\rho$ Lemma 5.2 i) holds with $\tilde{u}$ instead of $\hat{u}$ and we obtain

$$
\left|x_{s}(\tilde{u})-x_{s}(u)-d_{u} x_{s}(u) \cdot\left(\delta \tilde{w}_{0}^{s}, \delta w_{1}\right)\right|=o\left(\|\delta w, s\|_{W}\right) .
$$

Moreover, by (10.8) it holds

$$
\left\|\delta \tilde{w}_{0}^{s}-\delta w_{0}^{s}\right\|_{1} \leq\left\|\left(\delta \tilde{w}_{0}^{s}, \delta w_{1}\right)-\left(\delta w_{0}^{s}, \delta w_{1}\right)\right\|_{\mathcal{V}} \leq \sum_{i=1}^{N}\left|\left[w_{0}\left(x_{i}\right)\right]\right|\left|s_{i}\right| \leq C\|(\delta w, s)\|_{W}^{2}
$$


and thus by the Lipschitz continuity of (5.1) also

$$
\left|x_{s}(\hat{u})-x_{s}(u)-d_{u} x_{s}(u)\left(\delta w_{0}^{s}, \delta w_{1}\right)\right|=o\left(\|(\delta w, s)\|_{W}\right)
$$

where we utilize that by Lemma 5.2

$$
\left|d_{u} x_{s}(u) \cdot\left(\delta \tilde{w}_{0}^{s}-\delta w_{0}^{s}, 0\right)\right| \leq\|p(0, \cdot)\|_{\infty}\left\|\delta \tilde{w}_{0}^{s}-\delta w_{0}^{s}\right\|_{1}=o\left(\|(\delta w, s)\|_{W}\right) .
$$

Finally, we have again by Lemma 5.2 with $\bar{s}_{k}$ according to (10.6)

$$
\left|d_{u} x_{s}(u) \cdot\left(\delta w_{0}^{s}, \delta w_{1}\right)-\bar{s}_{k}\right| \leq \sum_{x_{i} \in I_{k}}\left[u_{0}\left(x_{i}\right)\right]_{+}\left|\int_{x_{i}}^{x_{i}+s_{i}}\right| p(0, x)-p\left(0, x_{i}\right)|d x|=o\left(\|s\|_{2}\right),
$$

where we have used that $p(0, x)$ is continuous in all admissible discontinuities $x_{i}$, cf. (7.12). This concludes the proof that

$$
\left(w_{0}, w_{1}, \sigma\right) \in W \longmapsto x_{s}\left(u^{s}\right)
$$

is Fréchet differentiable in 0 with derivative (10.6). We can now show that

$$
\left.\left(w_{0}, w_{1}, \sigma\right) \in W \longmapsto y\left(\bar{t}, \cdot ; u^{s}\right)\right|_{\hat{I}}
$$

is shift-differentiable at 0 . We know that (5.2) holds on $\|(w, \sigma)\|_{W}<\rho$. Hereby, the mappings $\bar{u} \in\left(\hat{V},\|\cdot\|_{\mathcal{V}}\right) \longmapsto Y_{\mp}\left(\bar{t}, \cdot,\left.\bar{u}_{0}\right|_{J \mp}, \bar{u}_{1}\right) \in C(\hat{I})$ are continuously differentiable. We have already observed that $\left.w_{0}^{s}\right|_{J_{\mp}}=\left.w_{0}\right|_{J_{\mp}}$ on $\|(w, \sigma)\|_{W}<\rho$ and deduce directly from (10.7) that

$$
\left(w_{0}, w_{1}, \sigma\right) \in W \longmapsto Y_{\mp}\left(\bar{t}, \cdot,\left.u_{0}^{s}\right|_{J_{\mp}}, u_{1}^{s}\right) \in C(\hat{I})
$$

are continuously differentiable on $\|(w, \sigma)\|_{W}<\rho$. According to Remark 4.3

$$
\left.\delta Y_{\mp} \stackrel{\text { def }}{=}\left(d_{u} Y_{\mp}(\cdot, u) \cdot\left(\left.\delta w_{0}\right|_{J_{\mp}}, \delta w_{1}\right)\right)\right|_{S_{\mp}}
$$

are on $S_{\mp}$ broad solutions of the variational equation (10.2) and satisfy on $J_{\mp}=S_{\mp}^{c l} \cap\{t=0\}$ the initial condition (10.3). Set now

$$
\delta y=\left.\delta Y_{-}(\bar{t}, \cdot)\right|_{x_{-}, x_{s}(u)[}+\left.\delta Y_{+}(\bar{t}, \cdot)\right|_{] x_{s}(u), x_{+}[}
$$

as claimed in (10.5). With $\left.\tilde{y}(\bar{t}, \cdot) \stackrel{\text { def }}{=} Y_{-}(\bar{t}, \cdot, \hat{u})\right|_{x_{-}, \bar{x}[}+\left.Y_{+}(\bar{t}, \cdot, \hat{u})\right|_{] \bar{x}, x_{+}[}$and $\hat{y}(\bar{t}, \cdot)=y(\bar{t}, \cdot ; \hat{u})$ we get by (10.12), (10.13) that $\|\tilde{y}(\bar{t}, \cdot)-y(\bar{t}, \cdot)-\delta y\|_{\infty, \hat{I}}=o\left(\|\delta w, s\|_{W}\right)$ and thus

$$
\begin{aligned}
& \left\|\hat{y}(\bar{t}, \cdot)-y(\bar{t}, \cdot)-S_{y(\bar{t}, \cdot)}^{\left(\bar{x}_{k}\right)}(\delta y, \bar{s})\right\|_{1, \hat{I}}= \\
& \quad=\left\|\hat{y}(\bar{t}, \cdot)-\tilde{y}(\bar{t}, \cdot)-\operatorname{sgn}\left(\bar{s}_{k}\right)[y(\bar{t}, \bar{x})]_{+} \mathbf{1}_{I\left(\bar{x}, \bar{x}+\bar{s}_{k}\right)}\right\|_{1, \hat{I}}+o\left(\|\delta w, s\|_{W}\right) .
\end{aligned}
$$

Using (5.2) and the definition of $\tilde{y}$ we obtain

$$
\left.\hat{y}(\bar{t}, \cdot)\right|_{\hat{I}}-\left.\tilde{y}(\bar{t}, \cdot)\right|_{\hat{I}}=\operatorname{sgn}\left(x_{s}(\hat{u})-\bar{x}\right)\left(Y_{-}(\bar{t}, \cdot, \hat{u})-Y_{+}(\bar{t}, \cdot, \hat{u})\right) \mathbf{1}_{I\left(\bar{x}, x_{s}(\hat{u})\right)}
$$

and since $Y_{\mp}(\bar{t}, \cdot, \cdot)$ are Lipschitz continuous at $(\bar{x}, u)$ by Lemma 4.1, we have

$$
\left\|Y_{-}(\bar{t}, \cdot, \hat{u})-Y_{+}(\bar{t}, \cdot, \hat{u})-[y(\bar{t}, \bar{x})]\right\|_{\infty, I\left(\bar{x}, x_{s}(\hat{u})\right)}=O\left(\|(\delta w, s)\|_{W}\right) .
$$

Oleinik's entropy condition yields $[y(\bar{t}, \bar{x})]=[y(\bar{t}, \bar{x})]_{+}$and thus we have by (10.15), (10.16)

$$
\begin{aligned}
& \left\|\hat{y}(\bar{t}, \cdot)-\tilde{y}(\bar{t}, \cdot)-\operatorname{sgn}\left(\bar{s}_{k}\right)[y(\bar{t}, \bar{x})]_{+} \mathbf{1}_{I\left(\bar{x}, \bar{x}+\bar{s}_{k}\right)}\right\|_{1, \hat{I}}= \\
& \quad=[y(\bar{t}, \bar{x})]_{+}\left|\bar{x}+\bar{s}_{k}-x_{s}(\hat{u})\right|+O\left(\|(\delta w, s)\|_{W}^{2}\right)=o\left(\|(\delta w, s)\|_{W}\right) .
\end{aligned}
$$


Inserting this in (10.14) yields the shift-differentiability of (10.10) on $\hat{I}$ with $\left(\left.\delta y\right|_{\hat{I}}, \bar{s}_{k}\right)=$ $T_{s, \hat{I}}(0) \cdot(\delta w, s)$ according to $(10.5),(10.6)$. As mentioned above shock points with a rarefaction wave as left and/or right state can be treated similar with Corollary 5.3.

Step 2: We show that (10.10) is even continuously shift-differentiable on $\left\{\|(w, \sigma)\|_{W}<\rho\right\}$ for sufficiently small $\rho>0$. In fact, on $\left\{\|(w, \sigma)\|_{W}<\rho\right\}$ the function on the right hand side of (10.10) is of the form (5.2). Fix an arbitrary $(w, \sigma) \in W,\|(w, \sigma)\|_{W}<\rho$, and consider the corresponding control

$$
\left(u_{0}^{s}, u_{1}^{s}\right)=u^{s} \stackrel{\text { def }}{=}\left(u_{0}+S_{u_{0}}^{\left(x_{i}\right)}\left(w_{0}, \sigma\right), u_{1}+w_{1}\right)
$$

Then $u_{0}^{s}$ is again piecewise $C^{1}$, and $u_{1}^{s}$ has the same regularity as $u_{1}$. Moreover,

$$
u_{0}+S_{u_{0}}^{\left(x_{i}\right)}\left(w_{0}+\delta w_{0}, \sigma+s\right)=u_{0}^{s}+S_{u_{0}^{s}}^{\left(x_{i}+\sigma_{i}\right)}\left(\delta w_{0}, s\right)
$$

i.e. varying $(\delta w, s)$ produces a shift variation of $u_{0}^{s}$. Thus, the arguments of Step 1 show that $(10.10)$ is also shift-differentiable at $(w, \sigma)$. As above, the shift-derivative $\left(\left.\delta \hat{y}^{s}\right|_{\hat{I}}, \bar{s}^{s}\right)=$ $T_{s, \hat{I}}(w, \sigma) \cdot(\delta w, s)$ on $\hat{I}$ is given by (10.5), (10.6) with $u, y, x_{i}$ replaced by $u^{s}, y\left(. ; u^{s}\right), x_{i}^{s}=$ $x_{i}+\sigma_{i}$. Consequently, it suffices to show the continuity of the shift-derivative in 0 . The continuity of (10.9) and (10.11) implies the continuity of $(w, \sigma) \longmapsto y\left(\bar{t}, x_{s}\left(u^{s}\right) \pm ; u^{s}\right)$ for $u^{s}$ according to (10.17). It remains to show that $(w, \sigma) \in W \longmapsto T_{s, \hat{I}}(w, \sigma) \in \mathcal{L}\left(W, L^{r}(\hat{I}) \times \mathbb{R}\right)$ is continuous for some $r>1$. Since (10.9) is continuous and (10.11) are continuously Fréchet differentiable, it is obvious from (10.12), (10.13) with $u^{s}$ instead of $u$ that the mapping $\left.(w, \sigma) \in W \longmapsto \delta \hat{y}^{s}\right|_{\hat{I}} \in L^{r}(\hat{I})$ depends continuously on $(w, \sigma) \in W$ in a neighborhood of 0 for all $r \in] 1, \infty[$.

Finally, using (10.6) and denoting the extreme backward characteristics of $y$ through $\left(\bar{t}, x_{s}(u)\right)$ by $\xi_{\mp}$ and of $y^{s} \stackrel{\text { def }}{=} y\left(\cdot ; u^{s}\right)$ through $\left(\bar{t}, x_{s}\left(u^{s}\right)\right)$ by $\xi_{\mp}^{s}$, we obtain

$$
\begin{aligned}
& \left|\bar{s}_{k}^{s}-\bar{s}_{k}\right| \leq\left\|p_{s}(0, \cdot)-p(0, \cdot)\right\|_{1, I_{k}}\left\|\delta w_{0}\right\|_{\infty, I_{k}}+\left(\left\|\xi_{-}-\xi_{-}^{s}\right\|_{\infty,[0, \bar{t}]}+\left\|\xi_{+}-\xi_{+}^{s}\right\|_{\infty,[0, \bar{t}]}\right) \\
& \quad \cdot\left(\left\|p_{s}(0, \cdot)\right\|_{\infty, J_{-} \cup J_{+}}\left\|\delta w_{0}\right\|_{\infty, J_{-} \cup J_{+}}+\left\|p_{s} g_{u_{1}}\left(\cdot, y^{s}, u_{1}^{s}\right)\right\|_{\infty, \Omega_{\bar{t}}}\left\|\delta w_{1}\right\|_{\infty, \Omega_{\bar{t}}}\right) \\
& \quad+\left\|p_{s} g_{u_{1}}\left(\cdot, y^{s}, u_{1}^{s}\right)-p g_{u_{1}}\left(\cdot, y, u_{1}\right)\right\|_{1, D_{k}}\left\|w_{1}\right\|_{\infty, D_{k}} \\
& \quad+\sum_{x_{i} \in I_{k}}\left(\left|\left[u_{0}^{s}\left(x_{i}^{s}\right)\right]_{+}-\left[u_{0}\left(x_{i}\right)\right]_{+}\left\|p_{s}\left(0, x_{i}^{s}\right)|+|\left[u_{0}\left(x_{i}\right)\right]_{+}\right\| p_{s}\left(0, x_{i}^{s}\right)-p\left(0, x_{i}\right)\right|\right)\left|s_{i}\right|
\end{aligned}
$$

where $p$ is the reversible solution of (10.4) and $p_{s}$ the reversible solution of (10.4) for $y^{s}$ instead of $y$ and $x_{s}\left(u^{s}\right)$ instead of $\bar{x}_{k}=x_{s}(u)$. We deduce

$$
\sup _{\|(\delta w, s)\|_{W}=1}\left|\bar{s}_{k}^{s}-\bar{s}_{k}\right| \rightarrow 0 \quad \text { as } \quad\|(w, \sigma)\|_{W} \rightarrow 0
$$

from the following observations. By (5.2) and the continuity of (10.9), (10.11) it follows that $\left\|\xi_{\mp}^{s}-\xi_{\mp}\right\|_{\infty,[0, \bar{t}]} \rightarrow 0$ as $\|(w, \sigma)\|_{W} \rightarrow 0$. Moreover, $y^{s}$ is bounded in $L^{\infty}$ with $y^{s} \rightarrow y$ in $L_{l o c}^{1}\left(\Omega_{T}\right)$ and exactly the same arguments as in the proof of Lemma $\left.5.2 \mathrm{i}\right)$ show with Theorem 6.10 that for all $\tau>0, R>0$ holds $p^{s}, p \in C^{0,1}([\tau, \bar{t}] \times[-R, R])$ and for all $r \in[1, \infty[$

$$
p^{s} \rightarrow p \text { in } C([\tau, \bar{t}] \times[-R, R]) \cap C\left([0, \bar{t}] ; L_{l o c}^{r}(\mathbb{R})\right) \text { as }\|(w, \sigma)\|_{W} \rightarrow 0 .
$$

In particular, we obtain $\left\|p^{s} g_{u_{1}}\left(\cdot, y^{s}, u_{1}^{s}\right)-p g_{u_{1}}\left(\cdot, y, u_{1}\right)\right\|_{1, D_{k}} \rightarrow 0$ by the Lebesgue dominated convergence Theorem and a subsequence-subsequence argument, since $g_{u_{1}}$ is by (A2) a Caratheodory function. To estimate the sum in the last term we observe that $\left[u_{0}^{s}\left(x_{i}+\sigma_{i}\right)\right]=$ $\left[u_{0}\left(x_{i}\right)\right]$ for $\|\sigma\|_{2}$ small enough. We still have to show that $\left|p_{s}\left(0, x_{i}+\sigma_{i}\right)-p\left(0, x_{i}\right)\right| \rightarrow 0$ if $\left[u_{0}\left(x_{i}\right)\right]_{+} \neq 0$. Remind that $p_{s}(0, \cdot), p(0, \cdot)$ are continuous in the admissible discontinuities $x_{i}+\sigma_{i}, x_{i}$ of $u_{0}^{s}$ and $u_{0}$, cf. (7.12). Fix an arbitrarily small $\tau>0$. We know that 
$p(\tau, \cdot) \in C_{l o c}^{0,1}(\mathbb{R})$ and that $\left\|p_{s}(\tau, \cdot)-p(\tau, \cdot)\right\|_{C([-R, R])} \rightarrow 0$. Let $\eta, \eta^{s}$ be the shocks of $y, y^{s}$ emanating from $x_{i}$ and $x_{i}+\sigma_{i}$, respectively. Clearly, for all $\tau>0$ sufficiently small $y(\tau, \cdot)$ satisfies the framework of Lemma 5.1 in a neighborhood of $\eta(\tau)$. Hence, we know from the first part of the proof that $\eta^{s}(\tau) \rightarrow \eta(\tau)$ as $\|(w, \sigma)\|_{W} \rightarrow 0 . p(t, \eta(t))$ and $p_{s}\left(t, \eta^{s}(t)\right)$ satisfy by Remarks 6.4, 6.6 an ordinary differential equation of the form (6.6). We thus obtain

$$
\begin{aligned}
\left|p_{s}\left(0, x_{i}^{s}\right)-p\left(0, x_{i}\right)\right| \leq & \left|p_{s}\left(\tau, \eta_{s}(\tau)\right)-p(\tau, \eta(\tau))\right| \\
& +\tau\left\|p g_{u_{1}}\left(\cdot, y, u_{1}\right)-p_{s} g_{u_{1}}\left(\cdot, y^{s}, u_{1}^{s}\right)\right\|_{\infty, D_{k}}
\end{aligned}
$$

and the right hand side becomes arbitrarily small for $\tau$ and $\|(w, \sigma)\|_{W}$ sufficiently small.

Step 3: It remains to consider the continuity points. Since $y(\bar{t}, \cdot ; u)$ contains no shock generation points, all continuity points satisfy the scenario of Lemma 4.4, 4.8, 8.1 or 9.1, respectively, corresponding to the cases $\mathrm{C}^{c}, \mathrm{R}^{c}, \mathrm{CB}^{c}$, and $\mathrm{RB}^{c}$. In case $\mathrm{C}^{c}$ there are by Lemma 4.4 ii) $\hat{I}=] x_{-}, x_{+}\left[\ni \bar{x}\right.$ and $z_{-}<\bar{z}<z_{+}$such that for $(Y, Z, V, S, J)=\mathrm{YC}\left(u, \bar{t},\left[z_{-}, z_{+}\right]\right)$ obtained by Lemma 4.1 holds

$$
\left.y(\bar{t}, \cdot ; \hat{u})\right|_{\hat{I}}=\left.Y\left(\bar{t}, \cdot ;\left.\hat{u}_{0}\right|_{J}, \hat{u}_{1}\right)\right|_{\hat{I}}
$$

for all $\hat{u} \in \hat{V}$ with $\hat{V}$ defined in Lemma 4.4 ii). Hereby, $x_{i} \notin J, i=1, \ldots, N$. Let $M_{\infty}>0$ be chosen large enough in the definition of $\hat{V}$. Then we find $\rho>0$ such that on $\left\{\|(w, \sigma)\|_{W}<\rho\right\}$ with $u^{s}$ in (10.7) holds $\left.u_{0}^{s}\right|_{J}=\left.\left(u_{0}+w_{0}\right)\right|_{J} \in C^{1}(J)$ and moreover $u^{s} \in \hat{V}$. Hence, (10.18) holds on $\left\{\|(w, \sigma)\|_{W}<\rho\right\}$ and it follows by Lemma 4.1 that

$$
\left.(w, \sigma) \in W \longmapsto y\left(\bar{t}, \cdot ; u^{s}\right)\right|_{\hat{I}} \in C(\hat{I})
$$

is continuously Fréchet differentiable on $\left\{\|(w, \sigma)\|_{W}<\rho\right\}$. From Lemma 4.1 we have with Remark 4.3 that

$$
\left.\delta Y \stackrel{\text { def }}{=}\left(d_{u} Y(\cdot, u)\left(\left.\delta w_{0}\right|_{J}, \delta w_{1}\right)\right)\right|_{S}
$$

is the broad solution of the linearized state equation (10.2) on $S$ for initial data (10.3). Hence, the derivative of (10.19) in 0 is given by (10.5).

In case $\mathrm{CB}^{c}$ Lemma 8.1 can be applied. Then $\bar{z}=x_{i}$ and $u_{0}$ is continuous (but not necessarily differentiable) in $x_{i}$. Let $\left.\hat{I}=\right] x_{-}, x_{+}\left[\ni \bar{x}, J\right.$ with $\bar{z}=\xi(0) \in J, S_{\mp}, Y_{\mp}$ and $\hat{V}$ be given according to Lemma 8.1. Then for $\rho>0$ sufficiently small we have $u^{s} \in \hat{V}$ on $\left\{\|(w, \sigma)\|_{W}<\rho\right\}$, since $\left.u_{0}^{s}\right|_{J}=\left.\left(u_{0}+w_{0}\right)\right|_{J} \in P C^{1}(J ; \bar{z})$. Hence,

$$
(w, \sigma) \in W \longmapsto y\left(\bar{t}, \cdot ; u^{s}\right) \in L^{r}(\hat{I})
$$

is by Lemma 8.1 Fréchet differentiable at 0 for all $r \in[1, \infty[$ and the derivative coincides with the derivative of $(w, \sigma) \in W \longmapsto \tilde{y}\left(\bar{t}, \cdot ; u^{s}\right) \in L^{r}(\hat{I})$ where $\tilde{y}$ is defined in (8.4). Using (8.4), the derivative of (10.20) is thus clearly

$$
d_{u} \tilde{y}(\bar{t}, \cdot ; u) \cdot\left(\left.\delta w_{0}\right|_{J}, \delta w_{1}\right)=\left.\delta Y_{-}(\bar{t}, \cdot)\right|_{] x_{-}, \bar{x}[}+\left.\delta Y_{+}(\bar{t}, \cdot)\right|_{] \bar{x}, x_{+}[} \stackrel{\text { def }}{=} \delta y
$$

where with $S_{\mp, \xi} \stackrel{\text { def }}{=} S_{\mp} \cap\{\mp(x-\xi(t))>0\}$ and the prolongations $u_{0}^{\mp}$ and $w_{0}^{\mp}$ as in (8.1)

$$
\delta Y_{\mp}=\left.\left(d_{u} Y_{\mp}\left(\cdot ;\left.u_{0}^{\mp}\right|_{J}, u_{1}\right) \cdot\left(\left.\delta w_{0}^{\mp}\right|_{J}, \delta w_{1}\right)\right)\right|_{S_{\mp, \xi}} .
$$

Since $\xi$ is a genuine forward characteristic of $y(\cdot, u)$ through $(0, \bar{z})$, it is clear that $\delta Y_{\mp}$ depend in $S_{\mp, \xi}$ only on the values of $u_{0}, \delta w_{0}$ on $J \cap\{\mp(x-\bar{z})>0\}$, respectively and are thus by Remark 4.3 on $S_{\mp, \xi}$ broad solutions of the linearized state equation (10.2) for initial values (10.3). Hence, $(10.21)$ is exactly (10.5) on $\hat{I}$. 
If $\bar{x}$ is a continuity point on a rarefaction wave (case $\mathrm{R}^{c}$ ) or on the boundary of a rarefaction wave (case $\mathrm{RB}^{c}$ ) then Lemma 4.8 or 9.1 is applicable. Now the Fréchet differentiability of (10.19) or (10.20) can be proven completely analogous. In case $\mathrm{R}^{c}(10.19)$ is continuously Fréchet differentiable on $\left\{\|(w, \sigma)\|_{W}<\rho\right\}, \rho>0$ small enough, in case $\mathrm{RB}^{c}$ at least (10.20) is continuously Fréchet differentiable for all $r \in[1, \infty[$. On the rarefaction wave the broad solution $\delta Y$ of (10.2) in (10.5) is now defined according to Remark 4.7 and satisfies thus the initial condition (10.3).

Combining now the shift-differentiability of (10.10) in a neighborhood of a shock point and the Fréchet differentiability of (10.19) or (10.20), respectively, in a neighborhood of continuity points, the shift-differentiability of (10.1) in 0 is obvious by selecting a finite covering of $I$. Moreover, we have shown that $(\delta y, \bar{s})=T_{s}(0) \cdot(\delta w, s)$ is actually given by $(10.5),(10.6)$. By using the local properties of the derivatives according to Lemmas 4.1 and 4.5 we conclude that $T_{s}(0) \in \mathcal{L}\left(W, P C\left(I ; \tilde{x}_{1}, \ldots, \tilde{x}_{\tilde{K}}\right) \times \mathbb{R}^{K}\right)$ with $\left(\tilde{x}_{k}\right)$ comprising the shock locations and the points of class $\mathrm{CB}^{c}$.

Consider finally the case that $u_{0}\left(x_{i}-\right) \neq u_{0}\left(x_{i}+\right)$ for all $x_{i}$. Then the scenario of Lemma 8.1, i.e. case $\mathrm{CB}^{c}$, does not occur. We have seen that (10.10) is continuously shiftdifferentiable and (10.19) in the cases $\mathrm{C}^{c}, \mathrm{R}^{c}$ or at least (10.20) for all $r \in[1, \infty$ [ in the case $\mathrm{RB}^{c}$ are continuously Fréchet differentiable on $\left\{\|(w, \sigma)\|_{W}<\rho\right\}, \rho>0$ sufficiently small. Hence, we obtain in fact continuous shift-differentiability of (10.1) on $\left\{\|(w, \sigma)\|_{W}<\rho\right\}$ by choosing a finite covering of $I$ by intervals $\hat{I}$.

REMARK 10.2. At the boundary of a rarefaction wave and along the backward characteristic through points $(\bar{t}, \bar{x})$ of class $\mathrm{CB}^{c}$ the broad solutions of the linearized equation according to Remark 4.3 or 4.7 can not be pieced together continuously. Nevertheless, the obtained broad solution is a weak solution across the jump, since obviously the jump condition is satisfied.

REMARK 10.3. With the proper definition of $f^{\prime}(y)$ along shocks weak solutions of (4.10) can also be defined on the domains $D_{k}$ confined by the extreme backward characteristics. However, since the broad solutions to both sides of a shock do in general not satisfy the jump condition across shocks, $\delta Y$ is a measure with singular part at shocks. This reflects the fact that the mapping $u \longmapsto y(\cdot ; u)$ is at best differentiable in $\mathcal{M}_{l o c}(\mathbb{R})-\mathrm{w}^{*}$. For the conservative case (i.e. $g_{y} \equiv 0, g_{u_{1}} \equiv 0$ ) the concept of duality solutions introduced in [1] yields solutions of this type for conservative transport equations with discontinuous coefficient. See also [11].

10.1. Differentiability of tracking-type functionals. Using Lemma 2.3 and Theorem 10.1 we get the Fréchet differentiability of a large class of tracking-type functionals (1.2).

THEOREM 10.4. Let the assumptions of Theorem 10.1 hold and let $J(y(\bar{t}, \cdot ; u))$ be defined as in (1.2) with $\phi \in C_{l o c}^{1,1}\left(\mathbb{R}^{2}\right)$ and $y_{d} \in L^{\infty}(I)$ being approximately continuous in $\bar{x}_{1}, \ldots, \bar{x}_{K}$. Then the functional

$$
\left(w_{0}, w_{1}, \sigma\right) \in W \longmapsto J\left(y\left(\bar{t}, \cdot ; u_{0}+S_{u_{0}}^{\left(x_{i}\right)}\left(w_{0}, \sigma\right), u_{1}+w_{1}\right)\right) \stackrel{\text { def }}{=} \hat{J}\left(w_{0}, w_{1}, \sigma\right)
$$

is Fréchet differentiable at 0 . The derivative can be computed by (2.2) with $\bar{y}=y(\bar{t}, \cdot ; u)$ and $\delta y, \bar{s}_{k}$ according to (10.5) and (10.6). Moreover, if $y_{d}$ is continuous in a neighborhood of $\bar{x}_{1}, \ldots, \bar{x}_{K}$ and if $x_{1}, \ldots, x_{N}$ are discontinuities of $u_{0}$ then the mapping is continuously Fréchet differentiable on $\left\{\|(w, \sigma)\|_{W}<\rho\right\}$ for $\rho>0$ sufficiently small.

11. Adjoint calculus for tracking-type functionals. Although the shift-differential is very useful for analytical purposes, the derivative of tracking-type functionals (1.2) can be much more conveniently computed via the adjoint state.

THEOREM 11.1. Let the assumptions of Theorem 10.4 hold and let $y_{d}$ be $P C^{1}$. Then

$$
d \hat{J}(0) \cdot(\delta w, s)=\left(p(0, \cdot), \delta w_{0}\right)_{2}+\left(p g_{u_{1}}\left(\cdot, y, u_{1}\right), \delta w_{1}\right)_{2, \Omega_{\bar{t}}}+\sum_{i=1}^{N} p\left(0, x_{i}\right)\left[u_{0}\left(x_{i}\right)\right]_{+} s_{i}
$$


where $p$ is the reversible solution according to Theorem 6.11 of the adjoint equation

$$
\begin{aligned}
& \partial_{t} p+f^{\prime}(y) \partial_{x} p=-g_{y}\left(t, x, y, u_{1}\right) p, \quad p(\bar{t}, \cdot)=p^{\bar{t}} \\
& p^{\bar{t}}(x)=\bar{\phi}_{y}(x) \stackrel{\text { def }}{=} \begin{cases}\int_{0}^{1} \phi_{y}\left(y(\bar{t}, x+)+\tau[y(\bar{t}, x)], y_{d}(x)\right) d \tau, & x \in I \\
0 & \text { else }\end{cases}
\end{aligned}
$$

With the domains $S_{k}, D_{k}$ from Theorem 10.1 holds $\left.p\right|_{D_{k}} \in C^{0,1}\left(D_{k} \cap\{t>\tau\}\right)$ for all $\tau>0$. Moreover $\left.p\right|_{S_{k}}$ is piecewise $C^{0,1}$ on $S_{k} \cap\{t>\tau\}$ for all $\tau>0$ with discontinuities along the backward characteristics emanating from discontinuities of $y_{d}$. This remains true for $\tau=0$ on all $D_{k}, S_{k}$ that contain no rarefaction wave.

Proof. All statements on the differentiability of $\hat{J}$ are a direct consequence of Theorem 10.4. It remains to justify (11.1). Let $p$ be the reversible solution of (11.2), (11.3) according to Theorem 6.11. $p$ is by Definition 6.5 and Remark 6.4 transported along the backward characteristics. Hence, the values of $p^{\bar{t}}$ on an interval $\left[x_{-}, x_{+}\right]$determine the values of $p$ in the domain confined by the minimal/maximal backward characteristic through $x_{\mp}$, respectively. Thus, $p$ vanishes outside the domain confined by the genuine backward characteristics through the continuity points $(\bar{t}, a)$ and $(\bar{t}, b)$. Moreover, $p$ coincides on each $D_{k}$ with the reversible solution of (11.2) for end data $p(\bar{t}, \cdot)=p^{\bar{t}}\left(\bar{x}_{k}\right)$ and is thus the reversible solution of Lemma 5.2 i) used in Theorem 10.1, (10.6) multiplied by $\bar{\phi}_{y}\left(\bar{x}_{k}\right)\left[y\left(\bar{t}, \bar{x}_{k}\right)\right]$, cf. (11.3). Hence, (2.2), (10.5), (10.6) and Theorem 10.1 yield

$$
\begin{aligned}
d \hat{J}(0) \cdot(\delta w, s)= & \left(p^{\bar{t}}, \delta y\right)_{2, I}+\sum_{i=1}^{N} p\left(0, x_{i}\right)\left[u_{0}\left(x_{i}\right)\right]_{+} s_{i} \\
& +\sum_{k=1}^{K}\left(\left(p g_{u_{1}}\left(\cdot, y, u_{1}\right), \delta w_{1}\right)_{2, D_{k}}+\left(p(0, \cdot), \delta w_{0}\right)_{2, I_{k}}\right)
\end{aligned}
$$

For the second term we have used that all admissible discontinuities $x_{i}$ are contained in the union of $I_{k}=D_{k}^{c l} \cap\{t=0\}, k=1, \ldots, K$.

We still have to rewrite the first expression on the right hand side by means of the adjoint state. Fix an arbitrary stripe $S_{k}$. Since $y$ is continuous on $S_{k}$, all characteristics are genuine. If $S_{k}$ contains backward characteristics emanating at $t=\bar{t}$ from the finitely many discontinuities of $y_{d}$ in $I$ or forward characteristics emanating from $\left(0, x_{i}\right)$ with $u_{0}\left(x_{i}-\right)=u_{0}\left(x_{i}+\right)$ these characteristics divide $S_{k}$ in finitely many substripes. Consider one of these substripes $S$. Then there are $x_{-}<x_{+}$such that $S$ is the domain confined by the maximal/minimal backward characteristic through $\left(\bar{t}, x_{\mp}\right)$, respectively, and Remark 4.3 applies with $Y=y(\cdot ; u)$ outside, Remark 4.7 with $Y_{r}=y(\cdot ; u)$ inside of rarefaction waves. By the choice of $S$ we have $y(\bar{t},),. y_{d} \in C^{0,1}(] x_{-}, x_{+}[)$and thus the function $p^{\bar{t}}$ in (11.3) is in $C^{0,1}(] x_{-}, x_{+}[)$. Since the reversible solution $p$ of (11.2) depends on $S$ only on $\left.p^{\bar{t}}\right|_{x_{-}, x_{+}[}$, we conclude exactly as in the proof of Lemma 5.2 that $\left.p\right|_{S} \in C^{0,1}(S \cap\{t>\tau\}) \cap C\left([0, \vec{t}], L_{l o c}^{2}(\mathbb{R})\right)$ for all $\tau>0$. Remarks 4.3 and 4.7 yield thus (4.11) for $D=S \cap\{t>\tau\}$. Since the $L^{2}$-traces of $p$ and $y$ exist at $t=0$ and $\left.p\right|_{S}$ satisfies (11.2) a.e. by Theorem 6.7, (4.11) gives for $\tau \rightarrow 0$

$$
\left(p^{\bar{t}}, \delta y\right)_{2,] x_{-}, x_{+}[}=\left(p(0, \cdot), \delta w_{0}\right)_{2, S^{c l} \cap\{t=0\}}+\left(p g_{u_{1}}\left(t, x, Y, u_{1}\right), \delta w_{1}\right)_{2, S} .
$$

The boundary terms along the confining characteristics $\xi_{\mp}$ drop out, since the outer normal $\left(n_{1}, n_{2}\right)^{T}$ is a multiple of $\left(f^{\prime}\left(y\left(t, \xi_{\mp}(t)\right)\right),-1\right)^{T}$. Summing over all stripes $S$ and inserting the result in (11.4) yields (11.1).

We already know from Theorem 6.10 that for all $\tau>0$ holds $\left.p\right|_{D_{k}} \in C^{0,1}\left(D_{k} \cap\{t>\tau\}\right)$ and that $\left.p\right|_{S_{k}}$ is piecewise $C^{0,1}$ on $S_{k} \cap\{t>\tau\}$ with discontinuities along the backward characteristics emanating from discontinuities of $y_{d}$ and that the same holds for $\tau=0$ whenever $S_{k}$ or $D_{k}$ contains no rarefaction wave. 
We finally show that the nondegeneracy assumptions of Theorem 10.1 hold for almost all $\bar{t} \in[0, T]$ under slightly stronger smoothness assumptions on $\left(u_{0}, u_{1}\right)$.

THEOREM 11.2. Let (A1)-(A3) hold and assume in addition that $f$ is $C^{3}$ and $g \in$ $L^{\infty}\left(0, T ; C_{\text {loc }}^{2}\left(\mathbb{R} \times \mathbb{R} \times \mathbb{R}^{m}\right)\right.$. If $u_{0} \in P C^{2}\left(\mathbb{R} ; x_{1}, \ldots, x_{N}\right), u_{1} \in L^{\infty}\left(0, T ; C_{\text {loc }}^{2}(\mathbb{R})^{m}\right)$ then the assumptions of Theorem 10.1 hold for almost all $\bar{t} \in[0, T]$.

Proof. By [8, Lem. 4.1, Cor. 4.2] the set of shocks is at most countable and for each shock $\eta$ the functions $t \mapsto y(t, \eta(t) \mp)$ are continuous outside of an at most countable set. Thus, for all except countably many times $\bar{t} \in] 0, T$ [ for all shocks $\eta$ the functions $t \mapsto y(t, \eta(t) \mp)$ are continuous at $\bar{t}$ and the extreme backward characteristics through $(\bar{t}, \eta(\bar{t}))$ do not propagate at the boundary of a rarefaction wave or reach $t=0$ in one of the points $x_{i}, i=1, \ldots, N$, where $u_{0}$ is continuous. Denote by $\left.B \subset\right] 0, T$ [ the set of all $\bar{t}$ having this property. Fix some $\bar{t} \in B$. If all shocks satisfy the framework of Lemma 5.1 or Corollary 5.3 then $y(\bar{t}, \cdot)$ has obviously only finitely many shocks on each compact interval $I$ and Theorem 10.1 is applicable.

Now assume that there exists a shock $\eta$ and a set $R \subset B$ with outer measure $\mu^{*}(R)>0$ such that $\eta$ does for all $\bar{t} \in R$ not satisfy the framework of Lemma 5.1 or Corollary 5.3. Fix an arbitrary density point $\hat{t} \in R$ of $R$ with respect to $\mu^{*}$.

Assume for concreteness that $\eta$ has no rarefaction wave as left or right state at time $\hat{t}$. Since $t \mapsto y(t, \eta(t) \mp)$ are continuous at $\hat{t}$, this holds also for all times $t$ in a sufficiently close neighborhood $S$ of $\hat{t}$ and since $\hat{t} \in R$ is a density point of $R$, we may reduce $R$ such that $R=$ $R \cap S$. By assumption (3.20) must be violated for all $\bar{t} \in R$ with $\bar{z}$ being one of the intersection points $z_{\mp}=\xi_{\mp}(0 ; \bar{t}, \eta(\bar{t}))$ of the minimal or maximal backward characteristic of $y$ through $(\bar{t}, \eta(\bar{t}))$ with $\{t=0\}$. Without restriction assume that for all $\bar{t} \in R$ (3.20) is violated on the left side of the shock, i.e. $z(\bar{t}) \stackrel{\text { def }}{=} \bar{z}=\xi_{-}(0 ; \bar{t}, \eta(\bar{t}))$. Then with $F(t, z) \stackrel{\text { def }}{=} \frac{d}{d z} \zeta\left(t ; z, u_{0}(z), u_{1}\right)$ we must have

$$
F(\bar{t}, z(\bar{t}))=0, \quad F(t, z(\bar{t}))>0, \quad 0 \leq t<\bar{t} \forall \bar{t} \in R .
$$

Since genuine backward characteristics may not intersect, the mapping $t \in S \mapsto z(t)$ is strictly monotone decreasing and thus almost everywhere differentiable. Without restriction, we may thus reduce $R$ such that $z(t)$ is differentiable in all $\bar{t} \in R$ and still $\mu^{*}(R)>0$. Now let $\tilde{t} \in R$ be a density point of $R$ with respect to $\mu^{*}$ and set $\tilde{z}=z(\tilde{t})$. Since $F(t, z)=$ $\delta \zeta\left(t ; z, u_{0}(z), u_{1} ; 1, u_{0}^{\prime}(z), 0\right)$ we obtain from (3.13) that $F$ is continuously differentiable w.r.t. $t$, and since $u_{0}$ is $C^{2}$ in a neighborhood of $\tilde{z}$ we conclude by using Lemma 3.3 and applying Proposition 3.2 to (3.13) that $(t, z) \mapsto \zeta\left(t ; z, u_{0}(z), u_{1}\right)$ is $C^{2}$ in a neighborhood $Q$ of $(\tilde{t}, \tilde{z})$. Moreover, we know from $\S 3.3$ that $\partial_{t} F(\tilde{t}, \tilde{z}) \neq 0$ and hence by (11.5) that $\partial_{t} F(\tilde{t}, \tilde{z})<0$, cf. also [8, Lem. 5.5]. Since $\tilde{t} \in R$ is a density point of $R$, there exists a strictly monotone increasing sequence $\left(t_{k}\right) \subset R$ with $t_{k} \nearrow \tilde{t}$. By the continuity of $t \mapsto y(t, \eta(t)-)$ in $\tilde{t}$ and the stability of genuine backward characteristics we have $z\left(t_{k}\right) \searrow z(\tilde{t})=\tilde{z}$ as $k \rightarrow \infty$ and thus eventually $\left(t_{k}, z\left(t_{k}\right)\right) \in Q$. Now we get by (11.5) and $\partial_{t} F(\tilde{t}, \tilde{z})<0$

$$
0=\lim _{k \rightarrow \infty} \frac{F\left(t_{k}, z\left(t_{k}\right)\right)-F(\tilde{t}, z(\tilde{t}))}{t_{k}-\tilde{t}}=\partial_{t} F(\tilde{t}, \tilde{z})+\partial_{z} F(\tilde{t}, \tilde{z}) \dot{z}(\tilde{t})<\partial_{z} F(\tilde{t}, \tilde{z}) \dot{z}(\tilde{t})
$$

and deduce from $\dot{z}(\tilde{t}) \leq 0$ that $\partial_{z} F(\tilde{t}, \tilde{z})=\left.\frac{d^{2}}{d z^{2}} \zeta\left(\tilde{t} ; z, u_{0}(z), u_{1}\right)\right|_{z=\tilde{z}}<0$. Hence, we obtain from $z\left(t_{k}\right) \searrow z(\tilde{t})=\tilde{z}$ that for all sufficiently large $k$

$$
\zeta\left(\tilde{t} ; z\left(t_{k}\right), u_{0}\left(z\left(t_{k}\right)\right), u_{1}\right)<\zeta\left(\tilde{t} ; z(\tilde{t}), u_{0}(z(\tilde{t})), u_{1}\right)=\eta(\tilde{t})
$$

i.e. the continuation of the genuine characteristic through $\left(t_{k}, \eta\left(t_{k}\right)\right)$ intersects the genuine backward characteristic through $(\tilde{t}, \eta(\tilde{t}))$ at some time $t \in] t_{k}, \tilde{t}[$ and must thus intersect the shock $\eta$ a second time on $] t_{k}, \tilde{t}$. This is impossible, since the angle between the genuine backward characteristics and $\eta$ is bounded away from zero independently of $k$ and also the 
curvature of the continuation of the characteristics is uniformly bounded. Hence, the assumption was wrong and the hypotheses of Lemma 5.1 can not be violated on a set of nonzero measure. Applying a similar argument in the case of a rarefaction wave as left or right state concludes the proof.

12. Conclusions and future work. We have presented a sensitivity calculus for entropy solutions of hyperbolic conservation laws with source terms that is based on a first order approximation by shift-variations. The obtained shift-differentiability result for the control-tostate mapping implies differentiability properties for a large class of tracking type functionals. For this class of functionals we have derived a gradient representation by using the adjoint state. Hereby, the adjoint state is the unique reversible solution of a transport equation with discontinuous coefficient that guarantees uniqueness only for the class of reversible solutions. These results can be used to state optimality conditions for the optimal control of flows with shocks and provide an analytical justification for the use of gradient based methods. In particular, it turns out that the numerical scheme used to compute the adjoint state should converge to the reversible solution in order to be consistent with the original problem. Since we allow shift-variations of the initial data, the shift-differentiability result can be repeatedly used over time slabs. We plan to exploit this in the design and analysis of a class of SQP methods with time domain decomposition.

Our analysis uses structural results for the state and the stability of reversible solutions of the adjoint equation with respect to its coefficients. This approach can formally be extended to systems and multidimensional problems and yields a correct sensitivity and adjoint calculus if the necessary stability properties of state and adjoint state actually hold.

The results of section 6 on the adjoint equation will be contained in a forthcoming paper. There we will also extend results of [1] on duality solutions for the corresponding forward problem to the case with source term which yields the correct linearization of the state equation.

We plan to use the adjoint calculus of this paper for the formulation of optimality conditions and the analysis of gradient based methods for the optimal control of flows with shocks. For the numerical approximation we plan to analyze which schemes for the discretization of the state equation lead to numerical adjoint schemes that converge to the correct reversible adjoint state thus yielding consistent gradient approximations. First results on the discretization of backward transport equations with discontinuous coefficients and the corresponding conservative forward problem were obtained for the case without source term by Gosse and James in the very recent paper [14].

Acknowledgments. A part of this research was done while the author was visiting the Department of Computational and Applied Mathematics and the Center for Research on Parallel Computation at Rice University. The author would like to thank John E. Dennis and Matthias Heinkenschloss for their hospitality and support.

I would like to thank my brother Michael, Technische Universität München, for helpful comments on an earlier draft of the paper and Matthias Heinkenschloss, Rice University, for interesting discussions on the material of the paper. Moreover, I am indebted to Günter Leugering, Universität Bayreuth, for his interest and support.

\section{REFERENCES}

[1] F. Bouchut, F. James, One-dimensional transport equations with discontinuous coefficients, Nonlinear Anal. 32 (1998), 891-933.

[2] - Differentiability with respect to initial data for a scalar conservation law, Hyperbolic problems: theory, numerics, applications, Vol. I (Zürich, 1998), 113-118, Internat. Ser. Numer. Math. 129, Birkhäuser, Basel, 1999.

[3] A. Bressan, G. Guerra, Shift-differentiability of the flow generated by a conservation law, Discrete Contin. Dynam. Systems 3 (1997), 35-58. 
[4] A. Bressan, A. Marson, A variational calculus for discontinuous solutions of systems of conservation laws, Comm. Partial Differential Equations 20 (1995), 1491-1552.

[5] E. M. Cliff, M. Heinkenschloss, A. R. Shenoy, An optimal control problem for flows with discontinuities, J. Optim. Theory Appl. 94 (1997), 273-309.

[6] - Adjoint-based methods in aerodynamic design-optimization, Computational methods for optimal design and control (Arlington, VA, 1997), 91-112, Progr. Systems Control Theory 24, Birkhäuser Boston, 1998.

[7] E. D. Conway, Generalized solutions of linear differential equations with discontinuous coefficients and the uniqueness question for multidimensional quasilinear conservation laws, J. Math. Anal. Appl. 18 (1967), $238-251$.

[8] C. M. DAFERmos, Generalized characteristics and the structure of solutions of hyperbolic conservation laws, Indiana Univ. Math. J. 26 (1977), 1097-1119.

[9] A. F. FILIPPov, Differential equations with discontinuous right-hand side, Amer. Math. Soc. Transl. Ser. 2, 42 (1964), 199-231.

[10] P. D. Frank, G. R. Shubin, A comparison of optimization-based approaches for a model computational aerodynamics design problem, J. Comput. Phys. 98 (1992), 74-89.

[11] E. Godlewski, P.-A. Raviart, The linearized stability of solutions of nonlinear hyperbolic systems of conservation laws. A general numerical approach, Math. Comput. Simulation, 50 (1999), 77-95.

[12] E. Godlewski, M. Olazabal, P.-A. Raviart, On the linearization of hyperbolic systems of conservation laws. Application to stability, Équations aux dérivées partielles et applications, 549-570, Gauthier-Villars, Éd. Sci. Méd. Elsevier, Paris, 1998.

[13] M. Golubitsky, D. G. Schaeffer, Stability of shock waves for a single conservation law, Advances in Math., 16 (1975), 65-71.

[14] L. Gosse, F. JAmes, Numerical approximations of one-dimensional linear conservation equations with discontinuous coefficients, to appear in Math. Comp. (2000); published electronically on March 1, 2000.

[15] D. HofF, The sharp form of Oleunik's entropy condition in several space variables, Trans. Amer. Math. Soc. 276 (1983), 707-714.

[16] F. James, M. Sepúlveda, Convergence results for the flux identification in a scalar conservation law, SIAM J. Control Optim. 37 (1999), 869-891.

[17] S. N. KRUŽKOV, First order quasilinear equations in several independent variables. Math. USSR Sbornik 10 (1970), 217-243.

[18] P. D. LAX, Hyperbolic systems of conservation laws II, Comm. Pure Appl. Math. 10 (1957), 537-566.

[19] $\mathrm{Ph}$. LeFloch, Z. P. XIN, Uniqueness via the adjoint problems for systems of conservation laws, Comm. Pure Appl. Math. 46 (1993), 1499-1533.

[20] O. A. OleiniK, Discontinuous solutions of nonlinear differential equations, Amer. Math. Soc. Transl. (2) 26 (1963), 95-172.

[21] E. Polak, Optimization. Algorithms and consistent approximations, Springer-Verlag, New York, 1997.

[22] J. SMOLler, Shock waves and reaction-diffusion equations, Springer-Verlag, New York-Berlin, 1983.

[23] E. TADMOR, Local error estimates for discontinuous solutions of nonlinear hyperbolic equations, SIAM J. Numer. Anal. 28 (1991), 891-906.

[24] S. UlBRICH, On the existence and approximation of solutions for the optimal control of nonlinear hyperbolic conservation laws, Optimal Control of Partial Differential Equations (Chemnitz, 1998), 287-299, Internat. Ser. Numer. Math. 133, Birkhäuser, Basel, 1999. 
Appendix. For convenience we present proofs of the existence and stability results for the adjoint equation that we stated in section 6 . They will be part of a forthcoming paper where we will also consider measure solutions for the corresponding conservative forward problem with source term.

One can show [1] that the generalized backward flow $X$ satisfies

$$
\left\|\partial_{s} X\right\|_{\infty, D_{b} \times \mathbb{R}} \leq\|a\|_{\infty},\left\|\partial_{t} X\right\|_{\infty \times \mathbb{R}, \stackrel{\circ}{D}_{b}} \leq\|a\|_{\infty} e^{\int_{0}^{T} \alpha},\left\|\partial_{x} X(s ; t, \cdot)\right\|_{\infty} \leq e^{\int_{t}^{s} \alpha}
$$

where $(s, t) \in D_{b}$ in the last inequality. Moreover,

$$
\left.\partial_{s} \partial_{x} X(s ; t, x) \leq \alpha(s) \partial_{x} X(s ; t, x) \quad \text { for a.a. } s \in\right] 0, T[.
$$

on $\stackrel{\circ}{D}_{b} \times \mathbb{R}$. Thus, for arbitrary $z_{1}<z_{2}$ and $0 \leq t \leq \sigma \leq s \leq T$

$$
X\left(s ; t, z_{2}\right)-X\left(s ; t, z_{1}\right) \leq X\left(\sigma ; t, z_{2}\right)-X\left(\sigma ; t, z_{1}\right)+\int_{\sigma}^{s} \alpha(\tau)\left(X\left(\tau ; t, z_{2}\right)-X\left(\tau ; t, z_{1}\right)\right) d \tau
$$

and hence

$$
X\left(s ; t, z_{2}\right)-X\left(s ; t, z_{1}\right) \leq\left(X\left(\sigma ; t, z_{2}\right)-X\left(\sigma ; t, z_{1}\right)\right) e^{\int_{\sigma}^{s} \alpha} \quad \text { for all } t \leq \sigma \leq s \leq T .
$$

Proof of Theorem 6.7: We show first that $p$ is well defined. Let $(t, x) \in \Omega_{T}$ be arbitrary. Since $z \longmapsto X(t ; 0, z)$ is surjective, there is $z \in \mathbb{R}$ with $x=X(t ; 0, z)$. Now (6.5) defines the values of $p$ on the curve $(s, X(s ; 0, z)), t \leq s \leq T$. If $z$ is not unique, then we get for all $\tilde{z}$ with $x=X(t ; 0, \tilde{z})$ by (12.3) $X(s ; 0, \tilde{z})=X(s ; 0, z)$ for all $s \in[t, T]$. Hence, the definition does not depend on the choice of $z$. As a simple consequence of (12.1), (6.5) we get (6.7). Hence, there exists a unique $p \in L^{\infty}\left(\Omega_{T}\right)$ satisfying (6.5). We show that $p$ is Lipschitzcontinuous. Let $z_{1}<z_{2}$ be arbitrary. Then $\Delta p(t) \stackrel{\text { def }}{=} p\left(t, X\left(t ; 0, z_{2}\right)\right)-p\left(t, X\left(t ; 0, z_{1}\right)\right)$ satisfies $\Delta p(T)=p^{T}\left(X\left(T ; 0, z_{2}\right)\right)-p^{T}\left(X\left(T ; 0, z_{1}\right)\right)$ and

$$
\frac{d}{d t} \Delta p(t)=-b\left(t, X\left(t ; 0, z_{2}\right)\right) \Delta p(t)-\left(b\left(t, X\left(t ; 0, z_{2}\right)\right)-b\left(t, X\left(t ; 0, z_{1}\right)\right)\right) p\left(t, X\left(t ; 0, z_{1}\right)\right) .
$$

Thus, setting $I(t)=\left[X\left(t ; 0, z_{1}\right), X\left(t ; 0, z_{2}\right)\right]$ we get for all $\tau \in[0, T]$

$$
\begin{aligned}
|\Delta p(\tau)| \leq & \int_{\tau}^{T}\|b(s)\|_{\infty, I(s)}|\Delta p(s)| d s+\left|p^{T}\left(X\left(T ; 0, z_{2}\right)\right)-p^{T}\left(X\left(T ; 0, z_{1}\right)\right)\right| \\
& +\int_{\tau}^{T}\|p(s)\|_{\infty, I(s)}\left|b\left(s, X\left(s ; 0, z_{2}\right)\right)-b\left(s, X\left(s ; 0, z_{1}\right)\right)\right| d s .
\end{aligned}
$$

Hence, we have by (12.3) with $\Delta X(t) \stackrel{\text { def }}{=} X\left(t ; 0, z_{2}\right)-X\left(t ; 0, z_{1}\right)$

$$
|\Delta p(\tau)| \leq\left(\left\|\partial_{x} p^{T}\right\|_{\infty}+\|b\|_{L^{1}\left(0, T ; C^{0,1}\right)}\|p\|_{\infty}\right) e^{\int_{\tau}^{T} \alpha} \Delta X(\tau)+\|b\|_{\infty} \int_{\tau}^{T}|\Delta p(s)| d s
$$

and by Gronwall for all $t \in[0, T]$

$$
|\Delta p(t)| \leq \Delta X(t)\left(\left\|\partial_{x} p^{T}\right\|_{\infty}+\|b\|_{L^{1}\left(0, T ; C^{0,1}\right)}\|p\|_{\infty}\right) e^{(T-t)\|b\|_{\infty}+\int_{t}^{T} \alpha}
$$

This yields a uniform bound for $\left\|\partial_{x} p\right\|_{\infty}$. Finally, let $z \in \mathbb{R}$ and $t_{1}, t_{2} \in[0, T], t_{1}<t_{2}$, be arbitrary then by (12.1)

$$
\begin{aligned}
& \left|p\left(t_{2}, X\left(t_{1} ; 0, z\right)\right)-p\left(t_{1}, X\left(t_{1} ; 0, z\right)\right)\right| \leq \\
& \leq\left|p\left(t_{2}, X\left(t_{2} ; 0, z\right)\right)-p\left(t_{1}, X\left(t_{1} ; 0, z\right)\right)\right|+\left|p\left(t_{2}, X\left(t_{2} ; 0, z\right)\right)-p\left(t_{2}, X\left(t_{1} ; 0, z\right)\right)\right| \\
& \leq\left(\|b\|_{\infty}\|p\|_{\infty}+\|a\|_{\infty}\left\|\partial_{x} p\right\|_{\infty}\right)\left|t_{2}-t_{1}\right| .
\end{aligned}
$$


Hence, $p \in C^{0,1}\left(\Omega_{T}\right)$. Finally, $p$ solves (6.1) a.e. in $\Omega_{T}$. In fact, for a.a. $(t, x)=(t, X(t ; 0, z))$ in $\Omega_{T}$ the Lipschitz-function $p$ is differentiable. Moreover, since $a(t, \cdot) \in B V_{l o c}(\mathbb{R})$ for a.a. $t$ by the one-sided Lipschitz condition, we have $a(t, x-)=a(t, x+)$ for a.a. $(t, x) \in \Omega_{T}$ and thus from (6.4) that

$$
\partial_{s} X(t ; 0, z)=a(t, X(t ; 0, z))
$$

for a.a. $(t, x)=(t, X(t ; 0, z))$. Now the chainrule yields with (6.5) that (6.1) is satisfied for all these $(t, x)$.

Since for $w \in C^{0,1}(\mathbb{R})$ holds $|w|_{v a r}=\left\|\partial_{x} w\right\|_{1}$, we obtain by summing (12.4) for suitable $z_{1}^{0}<z_{2}^{0}=z_{1}^{1}<z_{2}^{1}=\ldots$ that for all $0 \leq t \leq \tau \leq T$

$$
\begin{aligned}
\left\|\partial_{x} p(\tau)\right\|_{1, I(\tau)} \leq & \int_{\tau}^{T}\|b(s)\|_{\infty, I(s)}\left\|\partial_{x} p(s)\right\|_{1, I(s)} d s+\left\|\partial_{x} p^{T}\right\|_{1, I(T)} \\
& +\int_{\tau}^{T}\|p(s)\|_{\infty, I(s)}\left\|\partial_{x} b(s)\right\|_{1, I(s)} d s .
\end{aligned}
$$

With $I=I(t)$ and $J=\left[X\left(t ; 0, z_{1}\right)-\|a\|_{\infty}(T-t), X\left(t ; 0, z_{2}\right)+\|a\|_{\infty}(T-t)\right]$ holds by (12.1) $I(s) \subset J, t \leq s \leq T$, and by the Gronwall lemma follows

$$
\left\|\partial_{x} p(t)\right\|_{1, I} \leq\left(\left\|\partial_{x} p^{T}\right\|_{1, J}+\left\|\partial_{x} b\right\|_{1,[t, T] \times J}\|p\|_{\infty,[t, T] \times J}\right) e^{(T-t)\|b\|_{\infty,[0, T] \times J}}
$$

Since $p$ solves (6.1) a.e. in $\Omega_{T}$, we get for arbitrary $0<t_{1}<t_{2}<T$ and with $|I|$ denoting the length of $I$

$$
\begin{aligned}
\left\|\partial_{t} p\right\|_{1,\left[t_{1}, t_{2}\right] \times I} & \leq\|b p\|_{1,\left[t_{1}, t_{2}\right] \times I}+\|a\|_{\infty,\left[t_{1}, t_{2}\right] \times I}\left\|\partial_{x} p\right\|_{1,\left[t_{1}, t_{2}\right] \times I} \\
& \leq\left(t_{2}-t_{1}\right)\left(|I|\|b p\|_{\infty,\left[t_{1}, t_{2}\right] \times I}+\|a\|_{\infty,\left[t_{1}, t_{2}\right] \times I}\left\|\partial_{x} p\right\|_{L^{\infty}\left(t_{1}, t_{2} ; L^{1}(I)\right)}\right)
\end{aligned}
$$

Now (6.8) and the asserted properties of the constant $C$ follow directly from (6.7), (12.6), (12.7). Moreover, we see that $p \in H^{1,1}(] 0, T[\times I)$ with norm not depending on $\alpha$.

Proof of Theorem 6.8: Denote by $X$ and $X_{n}$ the backward flows according to Definition 6.3 for $a$ and $a_{n}$, respectively. By [1] holds $X_{n} \rightarrow X$ in $C\left(D_{b} \times[-R, R]\right)$ for any $R>0$. By the definition of reversible solutions we have for all $z \in \mathbb{R}$

$$
\begin{aligned}
p_{n}\left(T, X_{n}(T ; 0, z)\right) & =p_{n}^{T}\left(X_{n}(T ; 0, z)\right), \\
\frac{d}{d t} p_{n}\left(t, X_{n}(t ; 0, z)\right) & =-\left(b_{n} p_{n}\right)\left(t, X_{n}(t ; 0, z)\right) .
\end{aligned}
$$

For the reversible solution $p$ of (6.1) holds (6.5). Fix some $R>0$ and consider an arbitrary $(t, x) \in[0, T] \times[-R, R]$. Then there exist $z, z_{n} \in \mathbb{R}$ with $x=X(t ; 0, z)=X_{n}\left(t ; 0, z_{n}\right)$ and we have $X(s ; 0, z), X_{n}\left(s ; 0, z_{n}\right) \in\left[R-M_{a} T, R+M_{a} T\right] \stackrel{\text { def }}{=} J$ according to (12.1) for all $s \in[t, T]$ with an upper bound $M_{a}$ for $\left\|a_{n}\right\|_{\infty}$ and $\|a\|_{\infty}$. Since $X(s ; t, x)=X(s ; 0, z)$ and $X_{n}(s ; t, x)=X_{n}(s ; 0, z)$ by $(6.3)$, we have

$$
\begin{aligned}
p_{n}\left(T, X_{n}(T ; t, x)\right) & =p_{n}^{T}\left(X_{n}(T ; t, x)\right) \\
\frac{d}{d s} p_{n}\left(s, X_{n}(s ; t, x)\right) & \left.=-\left(b_{n} p_{n}\right)\left(s, X_{n}(s ; t, x)\right), s \in\right] t, T[
\end{aligned}
$$

and the same holds with $p, X, p^{T}, b$ instead of $p_{n}, X_{n}, p_{n}^{T}, b_{n}$. Therefore, the difference $\Delta p_{n}(s) \stackrel{\text { def }}{=} p(s, X(s ; t, x))-p_{n}\left(s, X_{n}(s ; t, x)\right)$ satisfies

$$
\begin{aligned}
\left|\Delta p_{n}(T)\right| & =\left|p^{T}(X(T ; t, x))-p_{n}^{T}\left(X_{n}(T ; t, x)\right)\right| \\
& \leq\left\|p^{T}-p_{n}^{T}\right\|_{C(J)}+\left\|\partial_{x} p^{T}\right\|_{\infty, J}\left\|X-X_{n}\right\|_{C\left(D_{b} \times J\right)}
\end{aligned}
$$


and for $s \in] t, T[$

$$
\begin{aligned}
\frac{d}{d s} \Delta p_{n}(s)= & \left(b_{n}\left(s, X_{n}(s ; t, x)\right)-b(s, X(s ; t, x))\right) p(s, X(s ; t, x)) \\
& -b_{n}\left(s, X_{n}(s ; t, x)\right) \Delta p_{n}(s) .
\end{aligned}
$$

Thus, we get with $J_{T} \stackrel{\text { def }}{=}[0, T] \times J$

$$
\begin{aligned}
\left|\Delta p_{n}(s)\right| \leq & T\|p\|_{C\left(J_{T}\right)}\left(\left\|b_{n}-b\right\|_{L^{\infty}(0, T ; C(J))}+\left\|\partial_{x} b\right\|_{\infty, J_{T}}\left\|X-X_{n}\right\|_{C\left(D_{b} \times J\right)}\right) \\
& +\left|\Delta p_{n}(T)\right|+\int_{s}^{T}\left\|b_{n}\right\|_{L^{\infty}(0, T ; C(J))}\left|\Delta p_{n}(\tau)\right| d \tau
\end{aligned}
$$

and we deduce by the Gronwall lemma that

$$
\lim _{n \rightarrow \infty}\left\|p-p_{n}\right\|_{C([0, T] \times[-R, R])}=0 .
$$

$\square$ 